\title{
British Thoracic Society guideline for the use of long- term macrolides in adults with respiratory disease
}

\author{
David Smith, ${ }^{1}$ Ingrid Du Rand, ${ }^{2}$ Charlotte Louise Addy, ${ }^{3}$ Timothy Collyns, ${ }^{4}$ \\ Simon Paul Hart (1) , ${ }^{5}$ Philip J Mitchelmore (1), ${ }^{6,7}$ Najib M Rahman, ${ }^{8}$ Ravijyot Saggu ${ }^{9}$
}

\begin{abstract}
- Additional material is published online only. To view please visit the journal online (http://dx.doi.org/10.1136/ thoraxjnl-2019-213929).
\end{abstract}

${ }^{1}$ North Bristol Lung Centre Southmead Hospital, Bristol, UK ${ }^{2}$ Respiratory, Wye Valley NHS Trust, Hereford, UK ${ }^{3}$ Centre for Medical Education, Queens University Belfast, Regional Respiratory Centre, Belfast City Hospital, Belfast, UK ${ }^{4}$ Medical Microbiology, Leeds Teaching Hospitals NHS Trust, Leeds, UK

${ }^{5}$ Cardiovascular and Respiratory Studies, Hull York Medical School/University of Hull, Hull, UK

${ }^{6}$ Institute of Biomedical and Clinical Science, College of Medicine \& Health, University of Exeter, Exeter, UK

${ }^{7}$ Department of Respiratory Medicine, Royal Devon and Exeter Hospital, Exeter, UK ${ }^{8}$ Oxford Respiratory Trials Unit and Oxford NIHR Biomedical Research Centre, University of Oxford, Oxford, UK ${ }^{9}$ Pharmacy, University College London Hospitals NHS Foundation Trust, London, UK

Correspondence to Dr David Smith, Southmead Hospital, North Bristol Lung Centre, Bristol BS10 5NB, UK; david.smith@nbt.nhs.uk

\section{Linked}

- http://dx.doi.org/10.1136/ thoraxjnl-2019-214039

\section{Check for updates}

(C) Author(s) (or their employer(s)) 2020. No commercial re-use. See rights and permissions. Published by BMJ.

To cite: Smith D, Du Rand I, Addy $\mathrm{CL}_{\text {, et al. Thorax }}$

2020;75:370-404.

\section{SUMMARY OF RECOMMENDATIONS AND GOOD PRACTICE POINTS \\ Asthma \\ Recommendations}

- Oral macrolide therapy could be considered to reduce exacerbation frequency in adults (50-70 years), with ongoing symptoms despite $>80 \%$ adherence to high-dose inhaled steroids $(>800 \mu \mathrm{g} /$ day $)$ and at least one exacerbation requiring oral steroids in the past year. This recommendation reflects the population within the AMAZES RCT which represents the highest quality evidence of macrolide therapy leading to a significant reduction in exacerbations. (Conditional)

- Treatment with azithromycin should be considered for a minimum of 6-12 months to assess evidence of efficacy in reducing exacerbations. (Conditional)

- Oral macrolide therapy should not be offered as a way to reduce oral steroid dose; in some individuals, this may result as a consequence of a reduction in exacerbations or symptoms. (Strong)

\section{Good practice points}

$\checkmark$ Optimisation of other asthma therapies including establishing good adherence to inhaled therapies should be performed before considering a trial of oral macrolide therapy.

$\checkmark$ Referral to a respiratory specialist or specialist asthma service should be considered prior to initiation of macrolide therapy aimed at reducing exacerbation frequency.

$\checkmark$ For safety purposes, an ECG should be performed prior to initiation of macrolide therapy to assess QTc interval. If QTc is $>450$ $\mathrm{ms}$ for men and $>470 \mathrm{~ms}$ for women, this is considered a contraindication to initiating macrolide therapy. Baseline liver function tests should also be measured.

$\checkmark$ Patients should be counselled about potential adverse effects before starting therapy including gastrointestinal upset, hearing and balance disturbance, cardiac effects and microbiological resistance.

$\checkmark$ Microbiological screening of sputum before and during macrolide therapy may be clinically helpful in patients who are able to expectorate sputum. This would allow monitoring for development of resistance and detect changes in microbial growth to direct appropriate antibiotic therapy if required. However, the resource implications of this approach have not been assessed.

$\checkmark$ If oral macrolide therapy is considered, justification for ongoing treatment should be guided by clinical response based on specific outcome measures including exacerbation frequency, symptoms and quality of life assessed at baseline.

$\checkmark$ A risk:benefit profile should be considered in each individual if significant side effects from oral macrolide therapy develop. If gastrointestinal side effects occur at the higher dose of azithromycin (500 mg thrice weekly), a dose reduction to azithromycin $250 \mathrm{mg}$ thrice weekly could be considered if macrolide therapy has been of clinical benefit.

$\checkmark \quad$ Liver function tests should be checked 1 month after starting treatment and then every 6 months. An ECG should be performed 1 month after starting treatment to check for new QTc prolongation. If present, treatment should be stopped.

$\checkmark$ Symptom improvement with macrolide treatment may be minimal and not consistent across all people with asthma. If macrolide therapy is considered for symptom reduction, this should be for a defined period (6-12 months) and stopped if no symptomatic improvement is seen. Use of a validated symptom score, such as the ACQ, may be useful to help make this assessment less subjective.

$\checkmark$ If the desired clinical outcome is achieved, the possibility of breaks in therapy may be considered to reduce treatment burden for patients. It is unclear whether this may also reduce antimicrobial resistance rates.

Please see quick reference guide in online supplementary file 1.

\section{Bronchiectasis}

Recommendations

- Long-term macrolide treatment could be offered to reduce exacerbations in those with high exacerbation rates (ie, 3 or more per year). (Strong)

- The dosing regimens with the greatest supportive evidence, when using macrolides to reduce exacerbation rates, are azithromycin $500 \mathrm{mg}$ three times a week, azithromycin 250 $\mathrm{mg}$ daily and erythromycin ethylsuccinate 400 $\mathrm{mg}$ twice a day. A starting dose of azithromycin $250 \mathrm{mg}$ three times a week could be used to 
minimise side effect risk with subsequent titration according to clinical response. (Conditional)

- When using macrolides to reduce exacerbation rates, therapy should be offered for a minimum of 6 months. (Strong)

- Macrolides can be considered with the aim of improving quality of life but may require a long period of therapy (eg, 1 year) for significant effects. (Conditional)

\section{Good practice points}

$\checkmark$ Therapies should be optimised in accordance with BTS Bronchiectasis Guidelines before considering long-term macrolide therapy (eg, airway clearance techniques and attendance at pulmonary rehabilitation courses).

$\checkmark$ Macrolides should only be started following discussion and shared decision-making between the patient and a respiratory specialist.

$\checkmark$ For safety purposes, an ECG should be performed prior to initiation of macrolide therapy to assess QTc interval. If QTc is $>450 \mathrm{~ms}$ for men and $>470 \mathrm{~ms}$ for women, this is considered a contraindication to initiating macrolide therapy. Baseline liver function tests should also be measured.

$\checkmark \quad$ Patients should be counselled about potential adverse effects before starting therapy including gastrointestinal upset, hearing and balance disturbance, cardiac effects and microbiological resistance. Microbiological assessment of sputum should be performed before therapy, including investigation for NTM. Macrolide monotherapy should be avoided if an NTM is identified. When evaluating for NTM infection, macrolides should not be used for 2 weeks before microbiological testing.

$\checkmark$ Accurate assessment of baseline exacerbation rate should be determined before starting long-term macrolides for bronchiectasis.

$\checkmark \quad$ Liver function tests should be checked 1 month after starting treatment and then every 6 months. An ECG should be performed 1 month after starting treatment to check for new QTc prolongation. If present, treatment should be stopped.

$\checkmark$ Subsequent follow-up at 6 months and 12 months should determine whether benefit is being derived from therapy. If there is no benefit, treatment should be stopped.

$\checkmark$ Even if benefit is seen, consideration should be given to stopping treatment for a period each year, for example, over the summer. Such a drug holiday may help with reducing the development of resistance while maintaining efficacy because the vicious cycle has been broken.

See quick reference guide in online supplementary file 1.

\section{Chronic obstructive pulmonary disease Recommendations}

- Long-term macrolide therapy could be considered for patients with COPD with more than three acute exacerbations requiring steroid therapy and at least one exacerbation requiring hospital admission per year to reduce exacerbation rate. (Conditional)

- Long-term macrolide therapy could be considered for a minimum of 6 months and up to 12 months to assess the impact on exacerbation rate. (Conditional)

\section{Good practice points}

$\checkmark$ Non-pharmacological and pharmacological therapies should be optimised prior to considering long-term macrolide therapy. This includes smoking cessation, optimised inhaler technique, optimised self-management care plan, airway clearance techniques and attendance at pulmonary rehabilitation courses.

$\checkmark \quad$ Macrolides should only be started following discussion and shared decision-making between the patient and a respiratory specialist.

$\checkmark$ For safety purposes, an ECG should be performed prior to initiation of macrolide therapy to assess QTc interval. If QTc is $>450 \mathrm{~ms}$ for men and $>470 \mathrm{~ms}$ for women, this is considered a contraindication to initiating macrolide therapy. Baseline liver function tests should also be measured.

$\checkmark$ Patients should be counselled about potential adverse effects before starting therapy including gastrointestinal upset, hearing and balance disturbance, cardiac effects and microbiological resistance.

$\checkmark$ Microbiological assessment of sputum should be performed before therapy, including investigation for NTM. Macrolide monotherapy should be avoided if an NTM is identified. Repeat assessments are recommended with clinical decline or during exacerbations to monitor resistance patterns.

$\checkmark$ Accurate assessment of baseline exacerbation rate should be determined before starting long-term macrolides for patients with COPD and a CT scan should be considered to exclude a possible diagnosis of bronchiectasis.

$\checkmark$ A risk:benefit profile should be considered in each individual if significant side effects from oral macrolide therapy develop. If gastrointestinal side effects occur at the higher dose of azithromycin (500 mg thrice weekly), a dose reduction to azithromycin $250 \mathrm{mg}$ thrice weekly could be considered if macrolide therapy has been of clinical benefit.

$\checkmark \quad$ Liver function tests should be checked 1 month after starting treatment and then every 6 months. An ECG should be performed 1 month after starting treatment to check for new QTc prolongation. If present, treatment should be stopped.

$\checkmark$ Subsequent follow-up at 6 and 12 months should determine whether benefit is being derived from therapy by using objective measures such as the exacerbation rate, CAT score or Quality of Life as measured by a validated assessment tool such as SGRQ. If there is no benefit treatment should be stopped.

$\checkmark \quad$ It is not necessary to stop prophylactic azithromycin during an acute exacerbation of COPD unless another antibiotic with potential to affect the QT interval has also been prescribed.

See quick reference guide in online supplementary appendix 1.

\section{Bronchiolitis obliterans (including post transplantation) Recommendations}

- Low-dose, long-term azithromycin (250 mg thrice weekly) could be considered to prevent the occurrence of BOS post lung transplantation. (Conditional)

- Low-dose azithromycin $(250 \mathrm{mg}$ alternate days for a trial period of 3 months) could be considered to treat BOS occurring in lung transplant recipients. (Conditional)

\section{Use of macrolides in other respiratory conditions \\ Cough \\ Recommendations}

- Long-term macrolide antibiotics should not be used to manage patients with unexplained chronic cough. (Conditional)

\section{Organising pneumonia}

There is insufficient evidence to make a recommendation. 


\section{Safety issues}

Gastrointestinal effects

Good practice points

$\checkmark$ Prior to initiating low-dose macrolide therapy, patients should be warned of the possibility of gastrointestinal side effects.

$\checkmark$ Gastrointestinal side effects may be ameliorated by dose reduction although this may also reduce clinical efficacy.

$\checkmark$ Clinicians should carefully consider the risk-to-benefit balance when considering therapy for those with pre-existing gastrointestinal symptomatology.

\section{Cardiac effects}

Good practice points

$\checkmark$ Prior to initiating low-dose macrolide therapy, patients should be asked if they have a history of heart disease, previous low serum potassium measurements, a slow pulse rate, a family history of sudden death or known prolonged QT interval. Patients with such a history should not receive low-dose macrolide therapy without careful consideration and counselling of the increased risk of adverse cardiac effects.

$\checkmark \quad$ Prior to initiating low-dose macrolide therapy, a drug history looking for agents that might prolong the QTc interval should be sought (see online supplementary appendices 3 and 4). Patients taking such agents should not receive lowdose macrolide therapy.

$\checkmark$ Prior to initiating low-dose macrolide therapy, an ECG should be performed to exclude a prolonged QTc interval defined as $>450 \mathrm{~ms}$ for men and $>470 \mathrm{~ms}$ for women (see methodology in online supplementary appendices 3 and 4). Patients with a prolonged QTc interval should not receive low-dose macrolides.

$\checkmark$ One month after initiating low-dose macrolide therapy, a second ECG should be performed to exclude the development of a prolonged QTc interval. Patients who develop a prolonged QTc interval on low-dose macrolides should stop the macrolide.

$\checkmark \quad$ If any new drug that could potentially prolong QTc time is started or if dose increases are made, repeat ECG assessment.

\section{Ototoxicity}

Good practice point

$\checkmark$ Prior to initiating low-dose macrolide therapy, patients should be asked if they have a history of hearing or balance difficulties. Such patients should be made aware of the potential for a further, almost always reversible, deterioratioGood practice pointsn in hearing or balance with macrolide therapy. Patients with pre-existing hearing or balance difficulties who wish to proceed with treatment should be asked to report any change in hearing or balance promptly.

\section{Other side effects}

Good practice points

$\checkmark \quad$ Prior to initiating low-dose macrolide therapy, baseline liver function tests (LFTs) should be checked.

$\checkmark \quad$ LFTs should be checked after 1 month of treatment and then every 6 months thereafter for the duration of therapy.

\section{Antimicrobial resistance}

Good practice points

$\checkmark \quad$ The risks associated with increasing antimicrobial resistance should be discussed with patients prior to starting low-dose macrolide therapy. Patients should understand the risk that there may not be an effective antibiotic for them, or someone else, when needed in the future. $\checkmark \quad$ Prior to initiating low-dose macrolide monotherapy, patients should be asked if they have a history of previous or current NTM infection or disease. Current NTM infection should be managed with reference to BTS guidance and precludes low-dose macrolide monotherapy. Successfully treated NTM disease should not preclude low-dose macrolide monotherapy.

$\checkmark$ If there is any clinical suspicion of possible NTM disease, patients should be screened via examination of sputum samples prior to starting therapy. If positive for recognised potential pathogenic species, low-dose macrolide prophylaxis is contraindicated.

\section{SECTION 1: INTRODUCTION \\ Aim of the guideline}

The aim of this guideline is to examine the evidence for the use of long-term, low-dose macrolide agents in the therapy of adult respiratory diseases and to develop guidance for clinicians in such use of these agents.

\section{Intended users of the guideline and target patient populations}

These guidelines will be of interest to UK-based clinicians caring for adults with respiratory disease including respiratory physicians, acute/general medicine physicians and respiratory specialist nurses. The guidelines may also be of interest to GPs, community matrons and practice nurses, hospice staff and community respiratory teams, physiotherapists, microbiologists, pathologists, pharmacists, haematologists and lung transplant teams.

\section{Scope of the guideline}

The Guideline Development Group (GDG) has examined the use of macrolides in adults ( $>16$ years) where the duration of treatment exceeds that usually employed to treat an acute infection and the dosage is less than that usually employed to treat an acute infection. Such usage is considered to be exerting an effect through mechanisms other than a direct antibacterial one, commonly described as immunomodulatory mechanisms. We have characterised this as long-term, low-dose usage and have examined such usage (often in comparison with conventional therapy) in the following conditions:

Asthma.

Bronchiectasis.

COPD.

Bronchiolitis obliterans.

Chronic cough.

Organising pneumonia.

Diffuse panbronchiolitis.

In addition, the GDG has looked at safety issues surrounding the long-term use of macrolides at both patient and population levels to help formulate pragmatic guidance in this area based on the best available evidence combined with clinical experience.

\section{Areas not covered by the guideline}

These guidelines exclude paediatric practice. The use of macrolides in cystic fibrosis has not been included recognising the parallel work of the National Institute for Health and Care Excellence (NICE) (Cystic fibrosis: long term azithromycin. Evidence Summary ESUOM37. November 2014) in this area. Long-term macrolides for chronic rhinosinusitis have not been included. 
The use of macrolides as antibacterial agents to treat respiratory infection is excluded.

\section{Limitations of the guideline}

Healthcare providers need to use clinical judgement, knowledge and expertise when deciding whether it is appropriate to apply recommendations for the management of patients. The recommendations cited here are a guide and may not be appropriate for use in all situations. The guidance provided does not override the responsibility of healthcare professionals to make decisions appropriate to the circumstances of each patient, in consultation with the patient and/or their guardian or carer.

The GDG recognises that none of the macrolide antibiotics are licensed in the UK for long-term low-dose usage as immunomodulatory agents at the time of guideline development.

\section{Members of the GDG}

The GDG was chaired by two respiratory consultants- Dr David Smith (DS) and Dr Ingrid Du Rand (IDR). The GDG had a wide membership and included colleagues from respiratory medicine, pharmacy and microbiology. A patient representative was on the group for the duration of the process. Those on the group were not required to be BTS members. Mrs Joan McCarthy was the lay representative.

A full list of members can be seen at online supplementary appendix 2 .

\section{Acknowledgements}

The GDG is grateful to the Standards of Care Committee for assistance during the development of the guideline, and particular thanks are due to Dr Toby Capstick and Dr Mike Crooks for their input and advice in relation to certain sections of the guideline.

\section{SECTION 2: METHODOLOGY OF GUIDELINE PRODUCTION}

\section{Establishment of guideline development group}

The GDG was convened in June 2016, with the first meeting taking place in October 2016. The full GDG met three times during the development of the guideline and kept in close contact by teleconference/WebEx and email throughout the process.

\section{Methodology}

This is the first BTS guideline to use the GRADE approach as part of the process of guideline development. Previous guidelines have used the SIGN methodology. BTS has made this change reflecting common practice in guideline development internationally across all medical specialities. The advantages of the GRADE approach are described in detail in the GRADE handbook and the BTS GRADE guideline production manual. GRADE specifies an approach to framing questions, choosing outcomes of interest and rating their importance, evaluating the evidence, and incorporating evidence with considerations of values and preferences of patients and society to arrive at recommendations. ${ }^{1}$ The methodology used to write the guideline adheres strictly to the criteria as set by the AGREE II collaboration, which is available online (www.agreetrust.org/resource-centre/agree-ii/). The British Thoracic Society Standards of Care Committee guideline production manual is available online (https://www.brit-thoracic.org.uk/ quality-improvement/guidelines/).
Table 1 Summary of outcome measures

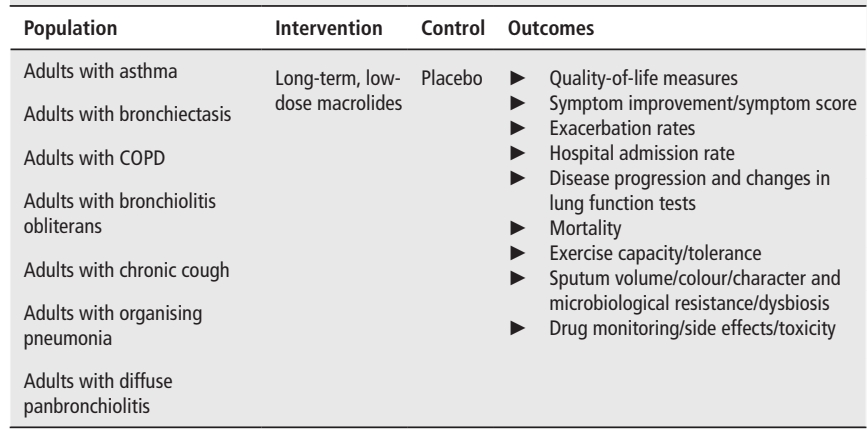

Summary of key questions, outcomes and literature search Clinical questions were formulated in the PICO (Patient, Intervention, Comparison and Outcome) format (see table 1). The key questions are summarised below.

1. Should long-term, low-dose macrolides be used in the treatment of adults with asthma?

2. Should long-term, low-dose macrolides be used in the treatment of adults with bronchiectasis?

3. Should long-term, low-dose macrolides be used in the treatment of adults with chronic obstructive pulmonary disease?

4. Should long-term, low-dose macrolides be used in the treatment of adults with bronchiolitis obliterans?

5. Should long-term, low-dose macrolides be used in the treatment of adults with chronic cough?

6. Should long-term, low-dose macrolides be used in the treatment of adults with organising pneumonia?

7. Should long-term, low-dose macrolides be used in the treatment of adults with diffuse panbronchiolitis?

The following patient-centred outcomes were identified by

the group when the scope of the guideline was agreed:

Quality-of-life measures.

Symptom improvement/symptom score.

Exacerbation rates.

Hospital admission rate.

Disease progression and changes in lung function tests.

Mortality.

Exercise capacity/tolerance.

Sputum volume/colour/character and microbiological resistance/dysbiosis.

Drug monitoring/side effects/toxicity.

The PICO framework was used to define the scope of the guideline and formed the basis of the literature search. The initial search was completed in February 2017 by York University. Systematic electronic database searches were conducted in order to identify all papers which might potentially be included in the guideline. For each question, the following databases were searched: Cochrane Database of Systematic Reviews (CDSR), Database of Abstracts of Reviews of Effects (DARE), Health Technology Assessment Database (HTA), Cochrane Central Register of Controlled Trials (CENTRAL), MEDLINE and MEDLINE In-Process, EMBASE and PubMed. The search strategy is available for review in online supplementary appendix 1 .

\section{Appraisal of the literature}

The literature search was run in 2016. The search was limited to papers published in English. The search identified a total of 5225 abstracts; after initial screening, this was reduced to 1152 .

Criteria formulated for initial screening of the abstracts were Whether the study addressed the clinical question. 
- Whether the appropriate study type was used to produce the best evidence to answer the clinical question.

- Abstract was in English.

- Studies in which macrolide therapy was used to treat acute respiratory tract infection were rejected.

- Abstracts were not rejected on the basis of the journal of publication, or the country in which the research was performed.

The full list of abstracts was retained and is kept in an archive. The literature search was repeated in December 2017 to capture additional published evidence while the guideline was in development prior to finalising the draft document. Additional references were included from critical appraisal of the literature and review of existing evidence base as appropriate.

Letters, conference papers and news articles were then removed -518 abstracts were rejected at this stage. IDR and DS read the remaining abstracts, marked those considered relevant to the scope of the guideline and allocated each relevant abstract to a clinical question(s). In total, 634 abstracts were allocated to clinical question(s).

The literature search was updated in late 2017 and identified 138 additional abstracts that were reviewed and 14 papers were selected to be critically appraised.

GDG members were allocated to work on individual questions in small groups.

Each abstract was read and at least two members agreed whether the paper was relevant, possibly relevant or irrelevant to the particular clinical question. Papers were excluded at the title/abstract sift if the following applied:

- If the paper did not address the clinical question and at least one of the outcomes concerned.

- If it was a case series of less than 20 patients-however, this was not an absolute cut-off. Professional judgement was applied such that some smaller case series were considered if evidence was sparse, and indeed some case reports of more than 20 patients were excluded if there was higher quality evidence available.

- If the language of the full paper was not English.

Full papers were obtained for all relevant, or possibly relevant, abstracts. Each paper was read in full and critically appraised by at least two members of the GDG to confirm relevance to the clinical question and the presence of at least one outcome of interest.

Each paper was then appraised by outcome(s), to generate a best estimate of the effect on each outcome and an index of the uncertainty associated with that estimate where possible. An evidence profile entry was completed for each outcome which included grading of the quality of the evidence. The type of evidence available for each outcome varied from systematic reviews through to case series; for each outcome, the highest quality evidence available was included. GRADEPro was used to generate the evidence profiles, published online on the BTS website where they are available for review (see online supplementary appendix 2). GRADEPro (https://gradepro.org/) is an easy-to-use, all-in-one web-based electronic guideline development tool to support guideline development using the GRADE process and methodology. In addition, it provides an electronic platform to present evidence tables, considered judgement and assessment of the evidence base.

The GRADE approach to rating the quality of evidence begins with the study design (table 2) and then, through a process of considered judgement, applies five reasons to possibly rate down the quality of evidence and three reasons to possibly rate up the quality (table 3).

\begin{tabular}{|c|c|c|}
\hline & Characteristics & Confidence \\
\hline High & $\begin{array}{l}\text { Based on consistent results from well- } \\
\text { performed randomised controlled trials }\end{array}$ & $\begin{array}{l}\text { Further research is very } \\
\text { unlikely to change the } \\
\text { estimate of the effect }\end{array}$ \\
\hline Moderate & $\begin{array}{l}\text { Based on randomised controlled trials } \\
\text { where there is evidence of bias or from } \\
\text { other well-conducted study types (eg, } \\
\text { well-executed observational studies) }\end{array}$ & $\begin{array}{l}\text { Further research is likely } \\
\text { to have an impact on the } \\
\text { estimate of the effect }\end{array}$ \\
\hline Low & $\begin{array}{l}\text { Based on observational evidence or } \\
\text { from controlled trials with several } \\
\text { serious limitations }\end{array}$ & $\begin{array}{l}\text { Further research is likely to } \\
\text { have an important impact }\end{array}$ \\
\hline Very low & Based on case studies or expert opinion & $\begin{array}{l}\text { Estimates of effect are far } \\
\text { from certain and more } \\
\text { research is needed }\end{array}$ \\
\hline
\end{tabular}

The GDG reviewed each clinical question during the regular meetings and consensus was reached.

In assessing the evidence the guideline development group combined low and very low evidence into one category (Low) as the body of evidence was limited.

\section{Development of recommendations}

Having generated evidence profiles for each of the clinical questions the GDG as a whole then considered the importance of each of the outcomes for each clinical question and proceeded to grade the overall body of evidence for critical and important outcomes.

The GDG went on to decide on the direction and strength of recommendations considering the quality of the evidence, the balance of desirable and undesirable outcomes and the values and preferences of patients and others. GRADE specifies two categories of strength of a recommendation as shown in table 4.

Good practice points (GPPs) were developed by consensus in areas where there was no quality evidence but the GDG felt that some guidance based on the clinical experience of the GDG might be helpful to the reader. These are indicated as shown below.

\section{$\sqrt{ }$ Recommended best practice based on the clinical experience of the guideline development group}

From the outset, it was acknowledged that there would be little high-quality evidence for some of the clinical questions identified. In this instance, low-grade evidence was considered, along with expert opinion via consensus at the meetings.

Cost-effectiveness was not considered in detail as in-depth economic analysis of recommendations falls outside of the scope of the BTS guideline production process. However, the GDG

\begin{tabular}{lc}
\hline Table 3 & Decreasing and increasing the grade of evidence \\
\hline $\begin{array}{l}\text { Decrease } \\
\text { grade if* }\end{array}$ & $\begin{array}{l}\text { Serious or very serious limitation to study quality } \\
\text { Important inconsistencies in results } \\
\text { Some or major uncertainty about directness of the evidence } \\
\text { Imprecise or sparse data (relatively few participants and/or events) } \\
\text { High probability of reporting bias }\end{array}$ \\
$\begin{array}{ll}\text { Increase } & \text { Magnitude of the treatment effect is very large and consistent } \\
\text { grade if } & \begin{array}{l}\text { Evidence of a large dose-response relation } \\
\text { All plausible confounders/biases would have decreased the } \\
\text { magnitude of an apparent treatment effect }\end{array}\end{array}$ \\
\hline
\end{tabular}

*Each quality criterion can reduce the quality by one or, if very serious, by two levels. See BTS GRADE guideline production manual for further details (https:// www.brit-thoracic.org.uk/quality-improvement/guidelines/). 
Table 4 Explanation of the terminology used in BTS recommendations

\begin{tabular}{lll}
\hline Strength & Benefits and risks & Implications \\
\hline Strong. It is recommended and so 'offer' & $\begin{array}{l}\text { Benefits appear to outweigh the risks (or vice versa) for the } \\
\text { majority of the target group }\end{array}$ & $\begin{array}{l}\text { Most service users would want to or should receive this } \\
\text { intervention }\end{array}$ \\
Conditional. It is suggested and so 'consider' & $\begin{array}{l}\text { Risks and benefits are more closely balanced, or there is more } \\
\text { uncertainty in likely service users values and preferences }\end{array}$ & $\begin{array}{l}\text { The service users should be supported to arrive at a decision } \\
\text { based on their values and preferences }\end{array}$ \\
\hline
\end{tabular}

were asked to be mindful of any potential economic barriers to the implementation of recommendations and GPPs.

Research recommendations were also highlighted and are listed in PICO format in an online supplementary appendix 3.

\section{Drafting the guideline}

The GDG corresponded regularly by email and meetings of the full group were also held in the period between July 2016 and early 2019. The guideline was discussed at an open session at the BTS Winter Meeting in December 2018. A revised draft guideline document was circulated to all the relevant stakeholders for consultation in December 2018 followed by a period of online consultation. The BTS SOCC reviewed the draft guideline in March 2018 and March 2019.

\section{Review of the guideline}

This guideline topic was chosen to inform practice in a growing clinical area, but also to act as a pilot for the use of GRADE methodology for all future BTS guidelines. The GDG recognise that the topic area would include a range of diseases with differing levels of evidence and thus act as a useful learning exercise for the introduction of GRADE methodology. It is not proposed to update this guideline as a distinct entity-the intention is for macrolide use to be encompassed in future diseasespecific guideline updates.

\section{Declarations of interests}

BTS Declarations of Interest forms have been completed by all members for each year they were part of the GDG. Details of these forms can be obtained from BTS Head Office. "Declarations of Interests" was a standing item at each GDG meeting.

\section{Stakeholders}

Stakeholders were identified at the start of the process. All stakeholder organisations were notified when the guideline was available for public consultation and a list is published in online supplementary appendix 2 .

\section{Other available guidance}

The GDG were aware of parallel workstreams within the BTS (eg, the BTS guideline for bronchiectasis in adults ${ }^{2}$ ) and in other organisations (eg, NICE) either complete or in progress at the time this guideline began development. Some of those in progress have subsequently been published and overlap with this guideline. The majority of these guidelines are disease specific rather than focusing on a single drug group. The pool of evidence available for these guidelines is the same, but the sample drawn from that pool is often slightly different. In addition, the methodology applied and the resources available to individual organisations are also variable. It is thus not surprising that the recommendations sometimes differ between contemporaneous documents. We have included a list of recently published guidelines and Cochrane reviews which overlap with this document.

BTS Guideline for Bronchiectasis in Adults $2019^{2}$
NICE CF diagnosis and management Oct $2017^{3}$

NICE Asthma diagnosis, monitoring and management Nov $2017^{4}$

NICE Chronic obstructive pulmonary disease (acute exacerbation): antimicrobial prescribing Dec $2018^{5}$

NICE Bronchiectasis (acute exacerbation): antimicrobial prescribing Dec $2018^{6}$

NICE COPD guideline $2018^{7}$

GOLD guideline COPD $2019^{8}$

ERS/ATS COPD Exacerbations $2017^{9}$

Cochrane Prophylactic antibiotic therapy for COPD $2018^{10}$

Cochrane Prolonged antibiotics for non-cystic fibrosis bronchiectasis in children and adults $2017^{11}$

Cochrane Antibiotics for exacerbations of asthma $2018^{12}$

Cochrane Macrolide antibiotics for bronchiectasis $2018^{13}$

Cochrane Macrolides for chronic asthma $2015^{14}$

GINA 2018 Global Strategy for Asthma Management and Prevention $^{15}$

Cochrane Adverse events in people taking macrolide antibiotics vs placebo for any indication $2019^{16}$

Within each of sections 4,5 and 6 , a summary of other guideline recommendations for that disease has been included.

\section{SECTION 3: INTRODUCTION TO THE USE OF MACROLIDES IN RESPIRATORY CARE}

The term macrolide describes compounds with a macrocyclic lactone ring of 12 or more elements. Within this definition are a variety of drugs including antibiotics, antifungals, prokinetic agents and immunosuppressants. The most commonly used macrolide antibiotics have 14-membered or 15 -membered lactone rings and include the first macrolide, erythromycin, which was launched commercially in 1952. Further 14-membered macrolides include clarithromycin and roxithromycin; the 15 -membered azithromycin became available in the 1980s. Macrolide antibiotics are active orally, have excellent tissue penetration and antimicrobial activity against many Grampositive bacteria, some Gram-negative organisms and some 'atypical' respiratory pathogens such as legionella and mycoplasma together with some mycobacterial species. This makes them a popular choice for respiratory tract infections.

Early studies with macrolide antibiotics in asthma in short antimicrobial courses ${ }^{17}$ and in longer, lower-dose regimens ${ }^{18}$ were the first to suggest a possible non-antimicrobial mode of action, possibly as a steroid-sparing agent.

Diffuse panbronchiolitis (DPB) was recognised as a distinct clinical entity in Japan in $1969^{19}$ having a poor prognosis, 25\% mortality at 10 years without Psendomonas aeruginosa infection or $>75 \%$ with $P$. aeruginosa. The first report of a dramatic response to low-dose, long-term treatment with erythromycin appeared in $1987^{20}$ and led to further confirmatory studies of macrolide efficacy in DPB. This acted as a catalyst for the subsequent exploration of the non-antimicrobial effects of macrolides. ${ }^{21}$ 
The mechanisms behind the immunomodulatory effects of macrolides have been widely researched. They include alterations in airway secretions through effect on ion transport and mucus production. Changes in the inflammatory process occur through effects on cytokine production, adhesion molecule expression and the functions of inflammatory cells, airway epithelial cells and other cells. Sublethal effects on bacteria include disruption of biofilms, interference with quorum sensing and reduced bacterial adherence, toxin production and mobility. ${ }^{22}$

The recognition of these immunomodulatory properties has led to the investigation of potential benefit from low-dose, longterm macrolides in a number of other chronic inflammatory airway diseases including cystic fibrosis, bronchiectasis, COPD, asthma, cryptogenic organising pneumonia (COP), chronic rhinosinusitis and bronchiolitis obliterans. In some of these conditions (eg, cystic fibrosis), the evidence base supporting the use of macrolides is large but in others (eg, COP) it is small.

Alongside this increase in the longer-term use of macrolides as immunomodulatory agents, concerns regarding the safety of these drugs used in this way have appeared. ${ }^{23}$ Gastrointestinal side effects are unsurprisingly common but rarely serious; however, ototoxicity and effects on myocardial tissue are potentially more harmful. Alterations in the microbiome of individuals and populations and the rapid emergence and persistence of macrolide-resistant organisms have also prompted discussion. ${ }^{24}$ It should also be noted that the rising rates of antimicrobialresistant pathogens is of global concern. Any consideration of the benefits of an individual patient receiving an antibiotic needs to be carefully balanced against the risk of increased resistanceboth for that individual patient but also for other individuals, both now and in the future.

It was against this background of expanding use of macrolides in inflammatory respiratory diseases that BTS decided to commission a guideline aimed at supporting best practice in a developing arena.

\section{SECTION 4: ASTHMA \\ Introduction}

The majority of asthma treatments aim to reduce airway inflammation with subsequent symptomatic improvement. Pathologically, asthma is characterised by airway inflammation and bronchial hyper-responsiveness. Airway inflammation can be predominantly neutrophilic or eosinophilic in nature. ${ }^{15}$ Despite an increase in therapeutic options and clear guidance on stepwise management, asthma symptoms can be difficult to manage and exacerbations remain a predominant feature for many. Adherence to treatment can be challenging. Newer therapeutic options, including biologics, can be expensive, require frequent monitoring and are limited to specific clinical phenotypes. ${ }^{1525}$

There has been significant interest in the potential role of oral macrolide therapy as an option in asthma. It is relatively inexpensive, easy to adhere to and has a physiologically relevant mechanism of action which may reduce airway inflammation in asthma. There is also evidence suggesting increasing asthma severity may be linked to chronic colonisation with Chlamydiae and Mycoplasma pneumoniae. It has therefore been postulated that benefit from macrolide therapy may also be through its antibacterial effects on these and other lung organisms. ${ }^{26}$

As a consequence, there have been a number of studies over several decades examining the response to macrolide therapy in asthma which vary considerably in design and outcome measures. The first study examining the role of macrolides in asthma was their use as a steroid-sparing agent in $1970 .{ }^{17}$ Studies since have examined their role in reducing airway inflammation and bronchial hyper-responsiveness, impact on symptoms and quality of life and most recently on their ability to reduce exacerbation frequency.

\section{Evidence base}

Examining the evidence base for macrolide therapy in asthma is particularly challenging because of the significant heterogeneity in study designs, outcome measures and study populations, as well as the heterogeneity of the asthma population itself. A Cochrane review of macrolide therapy in asthma identified significant publication bias, poor study design and lack of consistency in outcome measures as the key barriers to making recommendations based on the available evidence. ${ }^{14}$ Considering the size of the clinical population and burden of disease in this population, ${ }^{15}$ there is a relative paucity of high-quality evidence regarding macrolide use in asthma. There are relatively few studies with small study populations which are often underpowered to detect appropriate changes in the outcome measures used. The study populations are also varied limiting the applicability of their results to the wider asthma population and the ability to determine where macrolide therapy should sit within an asthma treatment regime.

Despite this, across multiple outcome measures statistical significance was achieved in many studies, but the magnitude of the changes seen were very small. The clinical significance of these changes varies from unknown to unlikely to be of any clinical benefit. This is reflected in the recommendations made in this guideline. No evidence of the impact of macrolide therapy on mortality, exercise capacity, disease progression or sputum production in people with asthma was found therefore no recommendations in regard to these outcomes can be made in this guideline. In all cases, macrolides should be considered after current therapy has been optimised and adherence established.

\section{Other recent guidance}

Recommendations on macrolide therapy are not included in current NICE/BTS/SIGN asthma guidelines. ${ }^{4} 25$ NICE guidance suggests referral to an asthma specialist and consideration of the addition of a trial of 'an additional drug' (which could include a macrolide) if asthma is uncontrolled in adults (aged 17 and over) on a moderate maintenance ICS dose with a LABA (either as MART or a fixed-dose regimen).

\section{Quality of life (QOL)}

QOL was the most consistently assessed outcome measure in studies of macrolide therapy in asthma. All but one study ${ }^{27}$ used the well-validated Asthma Quality of Life Questionnaire (AQLQ) score. This score was first validated in 1992 and has a minimal important difference (MID) of $0.5 .^{28}$ This is critical to appraising this body of evidence, as in similarity with the symptomatic improvements seen, after treatment with macrolides there is a consistent small but statistically insignificant $(p>0.05)$ improvement in QOL seen across the majority of studies. ${ }^{1427-36}$ In two studies comparing macrolide therapy with placebo, ${ }^{33} 34$ this change reached the MID of the AQLQ. The first used 8 weeks' treatment with clarithromycin ${ }^{34}$ and the second was the open-label treatment arm of a study of 48 weeks' treatment with azithromycin. ${ }^{33}$

It is therefore clear that treatment with macrolides may result in measurable improvements in QOL for people with asthma, but the clinical impact of these changes remains unknown and may be very small. 


\section{Symptoms}

Thirteen studies used symptom scoring as an outcome in response to macrolide therapy. ${ }^{14} 27$ 29-31 34-41 There was considerable variation in the scoring systems used, from the validated Asthma Control score (ACQ) to simplified unvalidated scoring systems, created specifically for an individual study. Overall, 10 of the studies demonstrated a reduction in symptoms. Three studies $^{29} 3133$ demonstrated an increase in symptoms, of which only one used a validated and reproducible symptom score. Although the majority of studies demonstrated an improvement, the actual changes were minimal, unlikely to be of clinical significance and reached statistical significance in only three cases. ${ }^{37} 38{ }^{41}$ None of the studies using the ACQ score demonstrated a change which would meet the ACQ minimal important difference (MID) (0.5). ${ }^{42}$

Further work is needed to see if these improvements may be of greater significance over more prolonged treatment periods, and whether symptomatic improvement is related to the impact of macrolide therapy on exacerbation rate.

\section{Exacerbations}

Exacerbations of asthma are defined as an increase in symptoms, accompanied by a reduction in peak flow rate (PEFR), of graded severity dependent on the degree of clinical and PEFR deterioration. ${ }^{425}$ Exacerbation rate is the definitive outcome measure in regard to the clinical efficacy of macrolides in other respiratory diseases, and the major purported driver for their use in cystic fibrosis, bronchiectasis and COPD. ${ }^{14}$ Two studies ${ }^{30} 40$ have been specifically designed to address this question in asthma. In other studies, exacerbations have been measured as a secondary outcome, with differing definitions between studies and insufficient statistical power to detect a reduction in exacerbation frequency. A meta-analysis of these studies showed exacerbations requiring steroids in $19.6 \%(31 / 158)$ of those treated with macrolides and $24.2 \%(32 / 132)$ of those not receiving macrolide treatment. ${ }^{14}$ However, this reduction was not sufficient to reach statistical significance $(\mathrm{p}>0.05)$.

Of the two studies ${ }^{30} 40$ specifically designed to determine a reduction in exacerbation frequency in response to azithromycin, the AMAZES trial demonstrated a significant reduction in exacerbations $(p<0.0001),{ }^{40}$ but the AZIZAST trial did not $(p=0.68) .^{30}$ This may reflect the duration of treatment (AMAZES=48 weeks; AZISAST $=6$ months), dose effect $($ AMAZES $=500 \mathrm{mg}$ thrice weekly; AZISAST $=250 \mathrm{mg}$ thrice weekly) or differing study populations. AMAZES recruited a larger population $(n=420)$ on high-dose inhaled steroids with an average of one exacerbation in the previous year, whereas the AZISAST population was smaller $(n=109)$ with higher inhaled steroid doses and two exacerbations in the previous year (see table 5). Both studies also had slightly differing definitions of an exacerbation. These studies are the highest quality evidence found for the purposes of this guideline and were suitably powered to detect a change in exacerbation frequency.

The AZISAST study demonstrated no significant difference between exacerbation rates with $48 \%(26 / 54)$ of the placebo group and $47 \%(26 / 55)$ of the azithromycin group experiencing an exacerbation (relative risk $0.98,95 \%$ CI 0.68 to 1.43 , $\mathrm{p}=0.68) \cdot{ }^{30}$ In AMAZES, 61\% (127/203) of the placebo group experienced an exacerbation compared with 44\% (94/213) of the azithromycin group giving an incidence rate ratio of 0.59 (95\% CI 0.47 to $0.74, \mathrm{p}<0.0001)^{40}$ (see table 5).

Both studies assessed whether the predominant type of lung inflammation impacted the effectiveness of macrolide therapy. In a predefined subgroup analysis of the AZISAST population, those with non-eosinophilic asthma showed a significant reduction in exacerbations in response to azithromycin treatment, with exacerbations occurring in 33\% (9/27) of the azithromycin group compared with $62 \%$ (18/29) of the placebo group (relative risk $0.54,95 \%$ CI 0.29 to $0.98, p=0.037) .{ }^{30}$ In AMAZES, both those with eosinophilic asthma and non-eosinophilic asthma demonstrated a reduction in exacerbations, with a slightly greater reduction seen in the eosinophilic group. The incidence rate ratio in the eosinophilic group was 0.52 (95\% CI 0.29 to $0.94, \mathrm{p}=0.030)$ and $0.66(95 \%$ CI 0.47 to $0.93, \mathrm{p}=0.019)$ in the non-eosinophilic group. ${ }^{40}$ This difference in outcomes may be partly explained by the differing definition of phenotypes between the two studies. In AZISAST, non-eosinophilic asthma was defined by a fraction of exhaled nitric oxide (FeNO) lower than the upper limit of normal and a blood eosinophilia $\leq 200 /$ $\mu \mathrm{L} .{ }^{30}$ In AMAZES, non-eosinophilic asthma was defined by a baseline sputum eosinophil count $<3 \%$ or blood eosinophil count $<300 / \mu \mathrm{L}$ if sputum unavailable. ${ }^{40}$

Overall, this evidence suggests that macrolide therapy may vary in its effectiveness between differing asthma phenotypes and that clinical benefit may be determined by more than the predominant type of inflammation. Further work is needed to investigate the impact of macrolides on exacerbation rate in differing asthma phenotypes and populations. However, it

Table 5 Comparison between the AZISAST and AMAZES trials

\begin{tabular}{|c|c|c|c|c|c|}
\hline & Population & Intervention & Follow-up & $\begin{array}{l}\text { Patients } \\
\text { intervention/control }\end{array}$ & Primary outcomes \\
\hline $\begin{array}{l}\text { Brusselle et al } \\
\text { (AZISAST) }^{30}\end{array}$ & $\begin{array}{l}18-75 \text { years } \\
\text { GOLD step } 4 / 5 \\
>1000 \mu \mathrm{g} \text { fluticasone or } \\
\text { equivalent } \\
2 \text { exacerbations requiring OCS } \\
\text { in past } 12 / 12 \\
\text { FeNO in normal limits }\end{array}$ & $\begin{array}{l}\text { Azithromycin } 250 \mathrm{mg} \\
\text { thrice weekly }\end{array}$ & 26 weeks & $\begin{array}{l}109 \\
55 / 54\end{array}$ & Exacerbation rate \\
\hline $\begin{array}{l}\text { Gibson et al } \\
(\text { AMAZES) })^{40}\end{array}$ & $\begin{array}{l}18 \text { years or older } \\
\text { Diagnosis of asthma (post- } \\
\text { bronchodilator reversibility } \\
>12 \% / \text { airway hyper- } \\
\text { responsiveness or PEFR } \\
\text { variability }>12 \% \text { ) } \\
\text { Partial loss of asthma control_- } \\
A C Q \geq 0.75\end{array}$ & $\begin{array}{l}\text { Azithromycin } 500 \mathrm{mg} \\
\text { thrice weekly }\end{array}$ & 48 weeks & $\begin{array}{l}420 \\
213 / 207\end{array}$ & Exacerbation rate \\
\hline
\end{tabular}

ACQ, Asthma Control Questionaire; GOLD, Global Initiative for Chronic Obstructive Lung Disease; OCS, Oral corticisteroids; PEFR, Peak Expiratory Flow Rate. 
appears from AMAZES ${ }^{40}$ that there is evidence of a reduction in exacerbations, but studies may need to run in larger populations for a longer duration to assess this effect successfully.

\section{Steroid dose reduction}

The original studies of macrolide use in asthma had the aim of reducing steroid dosage, ${ }^{17}$ but this has not been the stated outcome of a study using macrolide therapy since the 1990s. With the advent of newer biologic therapies and the focus on clinical phenotyping, there has been a move away from using therapies as steroid-sparing agents, towards a stratified approach to asthma therapy.

The three studies using steroid reduction as an outcome all used troleandomycin for between 2 and 52 weeks. ${ }^{43-45}$ All showed a reduction in steroid dosage, which has been confirmed in two Cochrane Reviews. ${ }^{14} 39$ However, these studies also included paediatric populations. Troleandomycin is also not widely used and is not available in the UK.

\section{Lung function/PEFR}

$\mathrm{FEV}_{1}$ remains a key outcome measure in trials of airways diseases. However, in asthma, $\mathrm{FEV}_{1}$ can show considerable variation and is used as a secondary outcome measure in many studies. Peak flow was used frequently as a primary and secondary outcome, but with considerable variation in timing, rigour of performance and units of measurement.

Overall $\mathrm{FEV}_{1}$ improved in response to oral macrolide therapy, but to a small degree which may not result in any clinical improvement. ${ }^{143135373946}$ This is illustrated by the results of four meta-analyses which individually compared $\mathrm{FEV}_{1}$ responses across 15 studies using a range of macrolides. The Cochrane meta-analysis demonstrated a $0.08 \mathrm{~L}(95 \%$ CI 0.02 to 0.14 $(\mathrm{p}=0.0097))$ improvement in $\mathrm{FEV}_{1}(47)$; the Tong et al metaanalysis showed a $0.11 \mathrm{~L}(95 \%$ CI 0.06 to $0.16(\mathrm{p}<0.001))$ improvement $^{36}$; and Reiter et al showed no effect on $\mathrm{FEV}_{1}$ (standardised mean difference (SMD) 0.05 (95\% CI -0.14 to 0.25 $(\mathrm{p}=0.60)) .{ }^{41} \mathrm{FEV}_{1}$ (\% predicted) was only analysed in the Tong et al meta-analysis, showing a SMD of $0.27 \%$ (95\% CI 0.05 to $0.59(\mathrm{p}=0.10)) .{ }^{36}$ The fourth meta-analysis was confined to studies examining troleandomycin, showing a $0.06 \mathrm{~L}$ (95\% CI -0.8 to $0.92(\mathrm{p}=0.88)$ ) change in $\mathrm{FEV}_{1} \cdot{ }^{39}$

Morning PEFR was the most commonly measured parameter across studies. A positive change in morning PEFR was seen in several studies, of a relatively smaller magnitude than that seen in $\mathrm{FEV}_{1}{ }^{14} 30353641$ The Cochrane and Tong et al meta-analyses showed a $2.22 \mathrm{~L} / \mathrm{min}(95 \% \text { CI } 9.73 \text { to } 14.17(\mathrm{p}=0.72))^{14}$ improvement and a SMD $0.25(95 \% \text { CI } 0.1 \text { to } 0.39(\mathrm{p}+0.001))^{36}$ in morning PEFR, respectively. A third meta-analysis by Reiter et al also showed a $6.7 \mathrm{~L} / \mathrm{min}(95 \%$ CI 1.35, $12.06(\mathrm{p}=0.014))$ improvement, but this analysis included paediatric study populations. ${ }^{41}$ The change in evening PEFR is more mixed across studies, with some showing a reduction ${ }^{35}$ rather than improvement, although with a smaller evidence base. ${ }^{14} 2930$ With the known variability of PEFR in asthma, again it is difficult to judge the clinical importance of these changes.

\section{Inflammation}

Studies examining the impact of oral macrolide therapy on inflammation in asthma have used a variety of outcome measures in both blood and sputum. In keeping with common clinical phenotypes, the impact of macrolides on both neutrophilic and eosinophilic inflammation has been assessed. Two meta-analyses ${ }^{14}$ examined the effect of macrolide therapy on sputum and blood eosinophil counts measured in three separate RCTs after treatment with roxithromycin, clarithromycin and azithromycin. Consistent reductions in blood eosinophils were shown (mean difference (MD) -33.5 (95\% CI -30.9 to $\left.-36.11) \times 10^{4} / \mathrm{mL}(\mathrm{p}<0.00001)\right)$, but only the study examining clarithromycin $\left(\mathrm{MD}-74 \times 10^{4} / \mathrm{mL}\right)$ demonstrated a reduction in sputum eosinophils. ${ }^{37}$

Five studies examined the impact of roxithromycin, ${ }^{46}$ clarithromycin $^{37}$ and azithromycin ${ }^{31}$ on sputum neutrophils. One study demonstrated an increase in sputum neutrophils (MD 19.2 (95\% CI -24.2 to 62.6$), \mathrm{p}>0.05)$ in response to azithromycin, ${ }^{47}$ but pooled results of the other studies, as part of a meta-analysis, demonstrated an overall reduction of $-0.25 \%$ (95\% CI -0.62 to $0.13, \mathrm{p}=0.20)^{36}$

Emerging biomarkers in the form of matrix metalloproteinase (MMP-9), neutrophil elastase and eosinophilic cationic protein (ECP) have also been examined. Clarithromycin resulted in a reduction in sputum MMP-9 and neutrophil elastase after 8 weeks of treatment. ${ }^{34}$ Serum and sputum ECP were both reduced after treatment with clarithromycin and roxithromycin. ${ }^{3746}$

FeNO is increasingly used as a biomarker of inflammatory activity in asthma and a measure of response to therapy. Three randomised trials used FeNO as an outcome measure all demonstrating a small and non-significant reduction in response to treatment with 12 weeks of azithromycin $(-1.94$ (95\% CI -5.97 to 2.10$) \mathrm{ppb}(\mathrm{p}=0.34)),{ }^{31} 26$ weeks of azithromycin (MD $-1.6 \mathrm{ppb}(\mathrm{p}=0.52))^{30}$ and 16 weeks of clarithromycin $(-4.6$ (SE $\pm 4.2) \mathrm{ppb}(\mathrm{p}=0.5))$, respectively. ${ }^{35}$

While there is considerable variation in study populations, treatment regimens and outcome measures, the overall picture is that macrolides do reduce airway inflammation in asthma. Further work is needed to clarify if this translates into clinically relevant improvements for patients and to determine which inflammatory biomarker may be of most use in determining response to treatment.

\section{Bronchial hyper-responsiveness}

Bronchial hyper-responsiveness has been used as an outcome measure in a number of studies of macrolide therapy in asthma ${ }^{313746}$ to provide proof of physiological effect and potential explanation for improvement in other clinical outcome measures. The studies confirm that macrolide therapy does result in improvements in bronchial hyper-responsiveness measured by methacholine challenge test (PC20/PD20). Two studies were specifically designed to assess bronchial hyper-responsiveness as a primary outcome. ${ }^{3748}$ When pooled, they demonstrated a 1.99 (95\% CI 0.46,3.52 $(\mathrm{p}=0.011)$ ) SMD improvement in methacholine response. A second meta-analysis of five studies demonstrated an improvement of 0.9 (95\% CI 0.5,1.75 $(p=0.04)) .{ }^{36}$ Other studies demonstrated varying magnitudes in the degree of improvement seen, likely related to differing macrolides, treatment dosages, duration of treatment, study populations and measurement differences. ${ }^{3135374648}$

While these findings support a physiological mechanism of action of macrolides through a reduction in airway inflammation and bronchial hyper-responsiveness, there seems to be a lack of clear correlation between the degree of improvement seen and any corresponding improvement in clinical outcomes. This requires further investigation in future trials.

\section{Microbiology}

Five studies specifically reported the impact of oral macrolide therapy on microbiological outcomes. Black et al conducted 
an RCT of 6 weeks of roxithromycin versus placebo in 232 people with asthma and raised $\operatorname{IgG} / \operatorname{IgA}$ antibody titres to Chlamydia pneumoniae. ${ }^{29}$ This demonstrated a reduction in IgG antibodies, but not IgA in response to treatment; but with no clinically significant changes in other outcome measures. Hahn et al demonstrated that high IgA but not IgG antibodies to C. pneumoniae were significantly associated with more severe asthma symptoms at the end of follow-up. ${ }^{33}$ Azithromycin resulted in improvements in IgA antibodies regardless of whether IgA antibody levels were high or low at the start of treatment. ${ }^{3.3}$

Sutherland et al attempted to conduct a PCR-stratified RCT based on PCR positivity for C. pneumoniae or Mycoplasma pneumoniae. ${ }^{35}$ Due to lower than anticipated numbers of PCRpositive participants, the two arms could not be successfully matched in number, but comparison of the two groups showed no significant differences in outcome between PCR positive $(\mathrm{n}=12)$ and PCR negative $(\mathrm{n}=80)$ after treatment with 16 weeks of clarithromycin. ${ }^{35}$

The AMAZES study monitored a subgroup of study participants for emergence of antimicrobial resistance to azithromycin within serial sputum samples over the 48 -week study period. ${ }^{40}$ At the study end, resistance to macrolides was found in 19/39 (48.7\%) receiving azithromycin and 12/42 (28.6\%) receiving placebo. ${ }^{40}$ AZISAST performed a similar subgroup analysis in 46 participants demonstrating an increase in macrolide-resistant streptococci from $47.8 \%$ to $87 \%$ participants in the azithromycin group and a reduction from $39.1 \%$ to $35 \%$ of participants in the placebo group. ${ }^{30}$ Interestingly, the percentage of macrolide-resistant streptococci reduced from $73.8 \%$ to $45.9 \%$ in the 4-week washout period at the end of the study. ${ }^{30}$ Further analysis of the oropharyngeal microbiome of a subgroup from AZISAST demonstrated a fivefold increase in Streptococcus salivarius and corresponding fivefold decrease in Leptotrichia wadei during azithromycin treatment. However, azithromycin had little impact on the rest of the microbiota. In keeping with the culture-based microbiology, the microbiome had already returned to pre-treatment levels in $50 \%$ participants by the end of the 4-week washout period. ${ }^{49}$

This suggests that chronic macrolide therapy may increase antimicrobial resistance to macrolides. This result has yet to be reproduced in other large studies and the clinical impact of this remains unknown. It is also unclear whether this increase in resistance is temporary or fluctuating. However, it does suggest that monitoring for antimicrobial resistance may be of benefit in those receiving chronic macrolide therapy. Use of breaks in chronic therapy, if the desired clinical outcome is achieved, may be considered to reduce resistance, as is the practice in some bronchiectasis services. Further work is needed to examine this relationship in more depth and determine the clinical impact of increasing resistance rates.

\section{Safety and adverse events}

Reporting of adverse events in the majority of studies was of low quality with no specific assessment for the well-documented adverse effects of macrolide therapy in the majority of studies. However, overall there was little evidence of significant reactions or side effects secondary to macrolide therapy in asthma. The most commonly reported side effects were gastrointestinal, including nausea and abdominal pain. In the meta-analysis performed by Reiter $e t a l$, a significantly increased risk of nausea was seen in those receiving macrolide therapy. ${ }^{41}$ Black et al also showed that $12.4 \%(13 / 105)$ of the roxithromycin group developed nausea compared with $4.5 \%(5 / 112)$ of the placebo group. $^{29}$

The AMAZES and AZISAST trials specifically assessed and reported adverse events as part of the planned study protocols. ${ }^{30}{ }^{40}$ In AMAZES, the overall serious adverse event (SAE) rate in the azithromycin group was $7.5 \%(16 / 213)$ compared with $12.8 \%(26 / 203)$ in the placebo group. ${ }^{40}$ Gastrointestinal side effects were more common in those on azithromycin compared with placebo-diarrhoea $(33.8 \%(72 / 213)$ vs $19.2 \%(39 / 203))$ and abdominal pain $(17.8 \%(38 / 213)$ vs $14.8 \%(30 / 203)) .{ }^{40}$ QTc prolongation was not seen more commonly in the azithromycin group with $0.5 \%$ (1 participant) of each group developing ECG changes during the study. ${ }^{40}$ There was also no difference in the rates of tinnitus $(0.9 \%(2 / 213)$ vs $1 \%(2 / 203))$ or hearing loss $(2.8 \%(6 / 213)$ vs $3.4 \%(7 / 203))$ seen. $^{40}$

In AZISAST, the SAE rate was identical in both placebo and azithromycin groups $(11 \%) .{ }^{30}$ Discontinuation of treatment was more common in the placebo group (9\% (5/54 vs 4\% (2/55)). Diarrhoea, nausea and abdominal pain were also more common in the placebo group. Two participants in the azithromycin group developed abnormal liver function tests, but the severity of this was not reported. No participants reported any change in their hearing. ${ }^{30}$

Overall, it appears that macrolides are well tolerated in people with asthma, although the quality of evidence is low and inclusion of formal assessment for adverse events should be included in future studies. There is a slightly higher incidence of gastrointestinal side effects which did not lead to cessation of treatment. Warning of the possibility of these side effects developing should be given to patients on initiation of treatment. There is insufficient evidence on the frequency of other adverse events specifically QTc prolongation, liver function abnormalities and audiological changes to make a formal recommendation on screening for these events. In general, participants with evidence of QTc prolongation or hearing loss were excluded from study populations further limiting the applicability of this evidence to the wider asthma population.

\section{Outcome weighting}

In the creation of this guideline, a set of key outcome measures in relation to macrolide therapy in management of respiratory disease were determined. However, this guideline's scope covers a spectrum of respiratory disease and the importance of individual outcome measures within each disease area varies. In regards to asthma, the GDG gave importance to those outcomes of most clinical relevance to respiratory physicians managing people with asthma and our recommendations reflect this. The group only made recommendations on these outcomes where the quality of evidence and magnitude of change was deemed sufficient to be of clinical utility. Therefore, recommendations are made in regard to exacerbation reduction, but not in relation to symptoms or quality of life as the changes seen in these outcomes were too small to be clinically important.

The body of evidence in regard to other outcomes, which may be of scientific or physiological relevance, is outlined in the evidence summary below, but no recommendations have been made in areas where the outcome is not used as a clinical endpoint—-for example, bronchial hyper-responsiveness.

\section{Evidence summary}

Oral macrolide therapy improves quality of life in people with asthma, but changes are small and their clinical significance is uncertain. (Moderate) 
Treatment with macrolides results in an improvement in asthma symptoms in some patients although this is small in magnitude and may not be applicable to all patients with asthma. (Low)

Oral macrolide therapy can reduce exacerbations of asthma in adults (50-70 years) with ongoing symptoms despite $>80 \%$ adherence to high-dose inhaled steroids $(>800 \mu \mathrm{g} /$ day $)$ and at least one exacerbation requiring oral steroids in the past year. (Low)

Azithromycin given thrice weekly at $500 \mathrm{mg}$ over 48 weeks results in a reduction in asthma exacerbations. (Low)

In some individuals, oral macrolide therapy may result in a reduction in oral steroid dose, but this is not a consistent finding. (Low)

Oral macrolide therapy can result in a small improvement in lung function and PEFR in people with asthma. (Low)

Oral macrolide therapy may reduce airway inflammation in asthma. (Low)

Oral macrolide therapy may reduce bronchial hyperresponsiveness in asthma. (Low)

Two studies demonstrated that treatment with oral macrolides (azithromycin) may result in an increase in bacteria within sputum resistant to macrolides, but currently there is no evidence of this adversely affecting clinical outcomes. (Low)

Oral macrolide therapy may result in an increase in gastrointestinal side effects including abdominal pain, nausea and diarrhoea. (Low)

There is insufficient evidence to make any recommendation on the impact of oral macrolide therapy on mortality, exercise capacity, disease progression or sputum characteristics in people with asthma.

\section{Recommendations}

- Oral macrolide therapy could be considered to reduce exacerbation frequency in adults (50-70 years), with ongoing symptoms despite $>80 \%$ adherence to high-dose inhaled steroids $(>800 \mu \mathrm{g} / \mathrm{day})$ and at least one exacerbation requiring oral steroids in the past year. This recommendation reflects the population within the AMAZES RCT which represents the highest quality evidence of macrolide therapy leading to a significant reduction in exacerbations. (Conditional)

- Treatment with azithromycin should be considered for a minimum of 6-12 months to assess evidence of efficacy in reducing exacerbations. (Conditional)

- Oral macrolide therapy should not be offered as a way to reduce oral steroid dose; in some individuals, this may result as a consequence of a reduction in exacerbations or symptoms. (Strong)

\section{Good practice points}

$\checkmark$ Optimisation of other asthma therapies including establishing good adherence to inhaled therapies should be performed before considering a trial of oral macrolide therapy.

$\checkmark$ Referral to a respiratory specialist or specialist asthma service should be considered prior to initiation of macrolide therapy aimed at reducing exacerbation frequency.

$\checkmark$ For safety purposes, an ECG should be performed prior to initiation of macrolide therapy to assess QTc interval. If QTc is $>450 \mathrm{~ms}$ for men and $>470 \mathrm{~ms}$ for women, this is considered a contraindication to initiating macrolide therapy. Baseline liver function tests should also be measured. $\checkmark$ Patients should be counselled about potential adverse effects before starting therapy including gastrointestinal upset, hearing and balance disturbance, cardiac effects and microbiological resistance.

$\checkmark$ Microbiological screening of sputum before and during macrolide therapy may be clinically helpful in patients who are able to expectorate sputum. This would allow monitoring for development of resistance and detect changes in microbial growth to direct appropriate antibiotic therapy if required. However, the resource implications of this approach have not been assessed.

$\checkmark$ If oral macrolide therapy is considered, justification for ongoing treatment should be guided by clinical response based on specific outcome measures including exacerbation frequency, symptoms and quality of life assessed at baseline.

$\checkmark$ A risk:benefit profile should be considered in each individual if significant side effects from oral macrolide therapy develop. If gastrointestinal side effects occur at the higher dose of azithromycin (500 mg thrice weekly) a dose reduction to azithromycin $250 \mathrm{mg}$ thrice weekly could be considered if macrolide therapy has been of clinical benefit.

$\checkmark$ Liver function tests should be checked 1 month after starting treatment and then every 6 months. An ECG should be performed 1 month after starting treatment to check for new QTc prolongation. If present, treatment should be stopped.

$\checkmark$ Symptom improvement with macrolide treatment may be minimal and not consistent across all people with asthma. If macrolide therapy is considered for symptom reduction, this should be for a defined period (6-12 months) and stopped if no symptomatic improvement is seen. Use of a validated symptom score, such as the ACQ, may be useful to help make this assessment less subjective.

$\checkmark \quad$ If the desired clinical outcome is achieved the possibility of breaks in therapy may be considered to reduce treatment burden for patients. It is unclear whether this may also reduce antimicrobial resistance rates.

See quick reference guide in online supplementary file 1.

\section{Research recommendations}

Further research, over a longer period, is needed to investigate the role of long-term macrolide therapy in reducing exacerbations of asthma.

Use of validated scoring systems and creation of a core outcome set should be considered in future trials of macrolide therapy in asthma to allow more accurate delineation of clinical response and comparison between studies.

Head to head comparisons of different macrolide therapy and of differing dose regimens for the same macrolide are required to optimise the use of macrolide therapy in asthma. Research into the discrepancy between significant reductions in inflammation but minimal improvements in clinical parameters is required.

Research into the impact of macrolide therapy in different clinical phenotypes of asthma should be performed in order to assess whether greater benefit is seen in one phenotype over another.

Research into the overlap between asthma and bronchiectasis and the impact of this on response to macrolides may assist in identifying asthma phenotypes which may respond differently to macrolide therapy. 


\section{SECTION 5: BRONCHIECTASIS Introduction}

Bronchiectasis is a chronic respiratory condition in which abnormally dilated bronchi are radiologically displayed in patients with a relevant clinical syndrome. These patients suffer from multiple symptoms, including a chronic productive cough, recurrent exacerbations associated with bronchial infection, dyspnoea and fatigue. While patients with cystic fibrosis can have both the radiological changes and the clinical syndrome, the term 'bronchiectasis' generally refers to those who do not have cystic fibrosis and the condition is often referred to as noncystic fibrosis bronchiectasis. Even after the exclusion of cystic fibrosis, a wide range of aetiologies are attributed to bronchiectasis, though a large proportion of cases are described as idiopathic or post-infective. ${ }^{50-54}$ Recent UK data report prevalence rates of 566/100 000 in adult women and 486/100 000 in adult men. ${ }^{53}$ Patients with bronchiectasis bear a significant burden of morbidity and mortality. ${ }^{5355}$

The basic concept for the management of bronchiectasis is to attempt to break the vicious recurrent cycle of chronic bacterial infection, inflammation, impaired mucociliary clearance and structural lung disease. ${ }^{56}$ With the goal of breaking this cycle and the extrapolation of management strategies from cystic fibrosis, it is unsurprising that macrolides have been used to try to improve outcomes in bronchiectasis. As well as having broad antimicrobial effects, anti-inflammatory effects are likely. Studies in patients with bronchiectasis have suggested reductions in Th17 cell responses ${ }^{57}$ and evidence of reduced airway inflammation via measuring markers such as IL8, neutrophil elastase and matrix metalloproteinase- $9 .^{58}$ A further attractive mechanism, particularly in a condition where $P$. aeruginosa is an important pathogen, is the inhibition of quorum sensing (a form of chemical signalling between bacteria). ${ }^{59}$

\section{Evidence base}

Davies and Wilson described early experiences of using azithromycin in $2004^{60}$ and subsequent published studies of varying quality, using different regimens, have added to the available evidence. The main body of high-level evidence is based on three RCTs ${ }^{61-63}$ (see table 6). These studies were performed in the Netherlands, Australia and New Zealand and were all placebo controlled. The macrolides used were azithromycin in the Netherlands and New Zealand studies, while the Australian study used erythromycin. Audit data suggest that azithromycin is the preferred macrolide in the UK at present. ${ }^{64}$ The two studies which used azithromycin had different dosing regimens (250 $\mathrm{mg}$ daily and $500 \mathrm{mg}$ three times a week). In addition, other studies of lower quality evidence have used other regimens such as azithromycin $250 \mathrm{mg}$ three times a week, ${ }^{606566}$ azithromycin $500 \mathrm{mg}$ twice weekly, ${ }^{67}$ erythromycin $500 \mathrm{mg}$ twice a week, ${ }^{68}$ clarithromycin $500 \mathrm{mg}$ once daily ${ }^{57}$ and roxithromycin 150 $\mathrm{mg}$ a day. ${ }^{58}$ The lack of consistency in the macrolide regimen used, and the absence of head-to-head comparisons, make the optimum macrolide and dosing unclear. ${ }^{2}$ Also, because none of the studies were longer than a year, the longer-term benefits and risks have not been established. Subsequent to the three RCTs which provide high-quality evidence, meta-analyses have been produced. ${ }^{69-71}$ While this provides pooled data, some lower quality evidence influence the output. ${ }^{6670} \mathrm{~A}$ further meta analysis of individual patient data (IPD) from the three main RCTs was published after the completion of literature searches and is referred to below because of the potential importance of its findings. ${ }^{72}$

\section{Other recent guidance}

The BTS bronchiectasis guidelines cover options beyond macrolides when considering long term antibiotic therapy in stable disease. ${ }^{2}$ They recommend macrolides as first choice for patients without $P$. aeruginosa with inhaled gentamicin as a second-line alternative. For patients with $P$. aeruginosa, the BTS guideline recommends inhaled colistin with macrolides as an alternative for those intolerant of inhaled antibiotics. Interestingly, the IPD meta-analysis by Chalmers et al suggests that macrolides are effective in patients with and without $P$. aeruginosa. ${ }^{72}$ In addition, the BTS guideline recommends a starting dose of azithromycin of $250 \mathrm{mg}$ three times a week to minimise side effects. While accepting that side effects are common with macrolides, we note that dropouts from studies due to side effects are rare and the strongest evidence of efficacy lies with higher dosing regimes. We suggest a pragmatic starting regime of azithromycin $500 \mathrm{mg}$ three times a week or $250 \mathrm{mg}$ daily in patients with bronchiectasis. However, if there is a patient history of drug intolerances, it would be sensible to start at the lower dose of $250 \mathrm{mg}$ three times a week.

Table 6 Three main randomised controlled trials of macrolides in bronchiectasis

\begin{tabular}{|c|c|c|c|c|c|c|}
\hline & Country & Population & Intervention & Follow-up & $\begin{array}{l}\text { Patients } \\
\text { Intervention/control }\end{array}$ & $\begin{array}{l}\text { Primary outcome } \\
\text { (exacerbations) }\end{array}$ \\
\hline $\begin{array}{l}\text { EMBRACE } \\
\text { Wong }\end{array}$ & New Zealand & $\begin{array}{l}\text { Mean age of } 60 \\
\text { At least one exacerbation requiring antibiotics } \\
\text { in last year } \\
\text { HRCT-defined bronchiectasis }\end{array}$ & $\begin{array}{l}\text { Azithromycin } 500 \mathrm{mg} \\
\text { three times a week }\end{array}$ & 6 months & $\begin{array}{l}141 \\
71 / 70\end{array}$ & $\begin{array}{l}\text { Exacerbations in first } 6 \\
\text { months: } 0.59 \text { (treatment } \\
\text { group) vs } 1.57 \text { (placebo } \\
\text { group) } p<0.0001\end{array}$ \\
\hline $\begin{array}{l}\text { BAT } \\
\text { Altenburg }\end{array}$ & Netherlands & $\begin{array}{l}\text { Mean age of } 62 \text { years } \\
\text { At least } 3 \text { LRTIs treated with antibiotics in the } \\
\text { last year } \\
\text { A sputum culture isolating a respiratory } \\
\text { pathogens in the last year } \\
\text { Bronchiectasis defined by HRCT or } \\
\text { bronchography }\end{array}$ & $\begin{array}{l}\text { Azithromycin } 250 \mathrm{mg} \\
\text { daily }\end{array}$ & 1 year & $\begin{array}{l}83 \\
43 / 40\end{array}$ & $\begin{array}{l}\text { Exacerbations in } 52 \text {-week } \\
\text { treatment period: } 0.84 \\
\text { (treatment group) vs } 2.05 \\
\text { (placebo group) } p<0.001\end{array}$ \\
\hline $\begin{array}{l}\text { BLESS } \\
\text { Serisier }\end{array}$ & Australia & $\begin{array}{l}\text { Mean age of } 62 \\
\text { At least two exacerbations requiring antibiotics } \\
\text { in the last year } \\
\text { Daily sputum production } \\
\text { Clinically stable for at least } 4 \text { weeks. HRCT- } \\
\text { defined bronchiectasis }\end{array}$ & $\begin{array}{l}\text { Erythromycin } \\
\text { ethylsuccinate } 400 \mathrm{mg} \\
\text { twice daily }\end{array}$ & 48 weeks & $\begin{array}{l}117 \\
59 / 58\end{array}$ & $\begin{array}{l}\text { Annualised exacerbation } \\
\text { rate: } 1.29 \text { (treatment } \\
\text { group) vs } 1.97 \text { (placebo } \\
\text { group) } p=0.003\end{array}$ \\
\hline
\end{tabular}


A NICE guideline was published in 2018 addressing acute exacerbations in bronchiectasis. ${ }^{6}$ With regards to preventing exacerbations, the document recommends the consideration of prophylactic antibiotics in people with repeated exacerbations. The guidance is less specific on which antimicrobials to use and what a significant exacerbation rate is. However, both the NICE and BTS documents advocate specialist input.

\section{Quality of life}

QOL data were collected from the three main RCTs and consequently there is high-level evidence available. While pooled data are also available, there are limitations of these data due to the influence of data of lower quality. ${ }^{66}$ The studies with the highest quality of evidence used the SGRQ to assess quality of life and any effect seen does not appear to be large. The BAT study did find a significant difference between treatment and placebo, with a reduction of -12.18 points after 1 year of treatment and a reduction of -4.12 in the placebo group $(p=0.046){ }^{62}$ A change of 4 points is considered clinically significant. In the treatment group, 64\% achieved this at 1 year while $46 \%$ did in the placebo group. While the other two main RCTs did not show a statistically significant improvement in SGRQ, the results from the BAT study should not be viewed as being inconsistent. In the EMBRACE study, 6 months of treatment failed to demonstrate a statistically significant difference though there was a trend towards benefit $(-5.17$ vs $-1.92,95 \%$ CI -7.21 to 0.72). ${ }^{61}$ Similar findings were also seen in the BLESS study with 48 weeks of therapy. ${ }^{63}$ This lack of clear effect may be due to a lack of sensitivity of the SGRQ for this type of therapy. Other studies have reported significant improvements in quality of life; however, the open-label nature or lack of a placebo group means they are of considerably lower quality of evidence. ${ }^{60}{ }^{66}$ Metaanalysis data did suggest a benefit with a change of -5.39 in the SGRQ; however, these data were influenced by a large change in a study of lower quality. ${ }^{69} 70$

On the basis of the current high-quality evidence, it appears that to obtain a significant improvement in quality of life, a long course of therapy may be required. On the basis of the BAT trial, this may need to be for a year. It is unclear what the impact of therapy beyond 1 year is. As the only high-quality evidence that suggests an improvement in quality of life is the BAT trial, the dosing regimen of azithromycin $250 \mathrm{mg}$ once a day carries the best evidence for achieving this outcome.

\section{Symptoms}

The prospective collection of symptom scores in RCTs has allowed the accumulation of some high-level evidence with regards to symptoms. This has been described in various ways including the symptom component of the SGRQ, the Leicester Cough Questionnaire and the lower respiratory tract infections-visual analogue scale (LRTI-VAS). The EMBRACE study demonstrated an improvement in the symptom component of the SGRQ with a difference in change from baseline of -6.7 (95\% CI -13.37 to -0.04). ${ }^{61}$ The BLESS study suggested a similar benefit, with a treatment effect of -5.3 reported; however, this did not reach statistical significance $(95 \% \mathrm{CI}-12.6$ to 2.1$) .{ }^{63}$ There was also no statistically significant change found in the Leicester Cough Questionnaire (LCQ). The BAT study used the LRTI-VAS and described a larger benefit in symptom improvement in those receiving treatment than those receiving placebo $(p=0.047) .{ }^{62}$ While not unanimous, there does appear to be some evidence of benefit with regards to symptoms, and inconsistencies may in part be due to differences and weaknesses in the scoring systems.

\section{Exacerbations}

Due to the three main RCTs using exacerbations as a primary endpoint and the subsequent meta-analyses, there is high level evidence which is in favour of using long-term macrolides to reduce exacerbations in bronchiectasis. ${ }^{61-63}$ 69-71 As in other respiratory conditions, there is variability in the interpretation of what exactly constitutes an exacerbation. A consensus definition has been published; however, this was subsequent to the important studies in this field. ${ }^{73}$

The previously mentioned three principal RCTs described benefit for this outcome when macrolides were used for between 6 and 12 months. ${ }^{61-63}$ The BLESS study described an incidence rate ratio of 0.57 (95\% CI 0.42 to 0.77 ) when compared with no intervention. ${ }^{63}$ The BAT study reported an absolute risk reduction of $33.5 \%$ (95\% CI $14.1 \%$ to $52.9 \%) .{ }^{62}$ Of interest in this study, there was an improvement in median number of exacerbation from 5 to 2 in the placebo group. Over a 6-month period, the EMBRACE study identified a rate ratio of 0.38 between the treatment group and the placebo group (95\% CI 0.26 to 0.54$){ }^{61}$ It is important to note that the EMBRACE study continued for a further 6 months after stopping the intervention and these benefits were maintained. However, it should also be noted that this study provided two definitions of 'exacerbations' and the results were not consistent, and that there was a higher baseline exacerbation rate in the control group. The meta-analysis data are consistent with an improvement in exacerbation rates. ${ }^{69-71}$

For macrolides to be used to reduce exacerbations, there needs to be a relevant exacerbation rate at baseline to consider when implementing this therapy. There were different entry criteria for the main trials with regards to baseline exacerbation rates. While entry into the EMBRACE study required just a single exacerbation for study recruitment, the mean exacerbation rate in the preceding year was over three (3.34 in treatment group and 3.93 in control group). In the BLESS and the BAT studies, two and three exacerbations were required, respectively, in the preceding year. In the BLESS study, approximately a third had five or more exacerbations in the preceding year, but it is unclear what the mean or median baseline exacerbation rate was. In the BAT study, the median baseline exacerbation rate was 4 (and 5 in the control group). In light of the baseline exacerbation rates in these trials, and three or more exacerbations being a marker of disease severity, ${ }^{74}$ a threshold of three or more exacerbations per year seems appropriate as a guide. It should be acknowledged that the aforementioned IPD meta-analysis that has followed the literature search for this guideline performed multiple subgroup analyses including for different exacerbation rates. One of these subgroups was those with a preceding exacerbation rate of less than this threshold (ie, 1 or 2 per year). While this group was small (37 receiving macrolides vs 36 receiving placebo), there appeared to be a benefit with regards to exacerbations, but a trend towards decreased QOL. It is conceivable that the benefits of macrolides may be outweighed by side effects in those with lower exacerbation rates. This subgroup require further study.

While out of keeping with the BTS Bronchiectasis Guideline and some UK respiratory clinicians' current practice, the regimens used in the three main RCTs (azithromycin $250 \mathrm{mg}$ daily, azithromycin $500 \mathrm{mg}$ three times a week and erythromycin ethylsuccinate $400 \mathrm{mg}$ twice a day) have the greatest supportive evidence of benefit. The BTS bronchiectasis guideline suggest a lower starting dose (azithromycin $250 \mathrm{mg}$ three times a week) noting the dose-related nature of side effects. Withdrawals due to side effects in the three main studies were, however, rare. It would seem sensible to start at the higher dose (azithromycin 
$500 \mathrm{mg}$ three times a week or $250 \mathrm{mg}$ daily) unless there is a history of previous drug intolerances. ${ }^{2}$ As well as variable dosing regimens, variable durations of therapy have also been studied. The highest quality studies used macrolide therapy for 6 or 12 months. While it is conceivable that it continues to provide benefit, there is no evidence either in favour or against the prolongation of therapy beyond 12 months.

\section{Exercise capacity}

Exercise capacity was measured by a 6 min walk test in both the BLESS and the EMBRACE studies. ${ }^{61}{ }^{63}$ This provides some high-level evidence via objective measures with blinded subjects and investigators. In neither study was there statistical evidence of benefit or harm from therapy. In addition, the activity component of the SRGQ was reported in the EMBRACE study ${ }^{61}$ and no impact on this outcome was seen. On the basis of this evidence from two studies, there is no indication that macrolides influence exercise capacity or tolerance.

\section{Sputum characteristics}

Sputum production is a common occurrence in many patients with bronchiectasis and is considered a significant issue by patients. ${ }^{75}$ There is, however, no high-quality evidence with regards to the effects of macrolide therapy on sputum. There is moderate level evidence of reduced sputum weight and sputum volume. ${ }^{6370}$ Estimates of the effect of therapy may be a reduction of approximately $11 \mathrm{~mL}$ per day $(95 \% \mathrm{CI}-12.70$ to -8.83$)$ based on pooled data. ${ }^{70}$ The BLESS study reported a treatment effect of a reduction of $4.3 \mathrm{~g}$ per day. ${ }^{63}$ It is unclear whether this scale of benefit is important to a patient but could be considered in discussion alongside assessment of other factors such as exacerbation rate when considering therapy.

\section{Safety and adverse events}

The evidence base for adverse events is quite variable within the bronchiectasis literature. While QTc prolongation is a potential concern with long-term macrolide use, there is very little evidence with regards to this adverse effect. In the BLESS study, QTc was monitored and one patient was withdrawn due to concerns with regards to prolongation, ${ }^{63}$ however pre-therapy and post-therapy ECGs suggested that this may not have been a drug-related effect. The EMBRACE and BAT studies did not address this potential adverse event. ${ }^{6162}$ An adverse impact on hearing is a further concern with macrolides; however, there is also limited evidence for this potential side effect. The BLESS and EMBRACE studies did not assess it, while the BAT study just performed a post-study questionnaire (and did not detect an effect). ${ }^{61-63}$

There is, however, considerably more evidence in these cohorts for gastrointestinal side effects. The meta-analysis pooled data show high-quality evidence for diarrhoea being an adverse event associated with long-term macrolide use. ${ }^{6971}$ The frequency of patients suffering diarrhoea was $19.3 \%-20.6 \%$ in the treatment groups compared with $4.1 \%-4.5 \%$ in the placebo groups. There was also high-level evidence for abdominal pain/ discomfort when reported in the BAT study $(18.6 \%$ vs $2.5 \%$, RR 7.44) and meta-analysis data (OR 6.97). ${ }^{62} 71$ Despite these gastrointestinal side effects, meta-analysis data assessing for withdrawal from studies due to side effects did not reveal any differences between those on macrolide or placebo (OR 1.18, 95\% CI 0.33 to 4.19$){ }^{69}$

While not a patient-centred outcome, high-quality evidence from the BLESS study reports increased micro-organism resistance. ${ }^{63}$ Further moderate level evidence was also added from the BAT study, though not the EMBRACE trial where routine testing was not performed. ${ }^{61}{ }^{62}$ In the BLESS study, there was a significant increase of macrolide resistance in commensal oropharyngeal streptococci with erythromycin use $(27.7 \%$ vs $0.04 \%, \mathrm{p}<0.001){ }^{63}$ The BAT study provided a significant amount towards the meta-analysis data and in the pathogens that were tested for macrolide sensitivity, $88 \%$ became resistant compared with $26 \%$ in the placebo group. ${ }^{62}$ At present, there is no evidence that the resistance seen either does or does not have an impact on clinical outcomes.

\section{Outcome weighting}

The available data allow assessment of the pre-specified outcomes with varying levels of evidence. Certain outcomes were excluded from our final assessment and recommendations. These included lung function, hospital admission rates, disease progression and death. Lung function was excluded despite available data, as the guideline group did not feel it is a useful measure when taken in the context of the current evidence. The trials were of too short a period for a meaningful change in lung function to be elicited despite statistically significant outcomes. For example, metaanalysis data from five studies described a statistically significant greater improvement of $\mathrm{FEV}_{1}$ of $0.02 \mathrm{~L}$ (mean weighted difference). ${ }^{70}$ This was not considered a clinically meaningful change for an individual patient. Hospital admission rate data were available; however, due to such low incidence rates for this outcome in the three RCTs which reported it, no recommendations are made for this outcome. ${ }^{61-63}$ The outcomes of disease progression and death are not commented on due to a lack of data. The outcomes of QOL, symptoms, exacerbation rates, exercise capacity, sputum characteristics and adverse events were all considered 'important' in the GRADE process.

\section{Evidence summary}

There is evidence of an improvement in QOL as measured by SGRQ when azithromycin $250 \mathrm{mg}$ daily is used for 1 year (High). Other high-quality studies with other dosing regimens showed a trend towards benefit but not a statistically significant benefit. Meta-analysis data reported a benefit but there are concerns regarding the inclusion of a study of lower quality (Low).

There is evidence from high-quality studies of improvement in symptom scores; however, inconsistency exists which may be due to different scoring systems being used. (Moderate)

Long-term macrolide treatment reduces exacerbations in bronchiectasis. (High)

When using macrolides to reduce exacerbation rates, the dosing regimens with the greatest supportive evidence are azithromycin $250 \mathrm{mg}$ daily, azithromycin $500 \mathrm{mg}$ three times a week and erythromycin ethylsuccinate $400 \mathrm{mg}$ twice a day. (Moderate)

Studies with other dosing regimens, including azithromycin $250 \mathrm{mg}$ three times a week (as pragmatically suggested in the BTS Bronchiectasis Guideline), have also reported a reduction in exacerbation, but have a lower evidence base. (Low)

The studies with the greatest evidence for reducing exacerbations used therapy for a minimum of 6 months. (High) The impact beyond 12 months is unknown.

There is evidence for a reduction in exacerbations over 12 months when therapy is used for 6 months and then not for the subsequent 6 months. (Moderate) It is unknown what the impact is of subsequently recommencing.

Long-term macrolide therapy is not associated with improved exercise capacity. (High) 
Long-term macrolide therapy may reduce sputum volume and weight. (Moderate)

Long-term macrolide therapy is associated with diarrhoea and with abdominal pain. (High)

Long-term macrolide usage can result in increased antimicrobial resistance. (High) It is unknown if this has a clinical impact.

\section{Recommendations}

- Long-term macrolide treatment could be offered to reduce exacerbations in those with high exacerbation rates (ie, 3 or more per year). (Strong)

- The dosing regimens with the greatest supportive evidence, when using macrolides to reduce exacerbation rates, are azithromycin $500 \mathrm{mg}$ three times a week, azithromycin $250 \mathrm{mg}$ daily and erythromycin ethylsuccinate $400 \mathrm{mg}$ twice a day. A starting dose of azithromycin $250 \mathrm{mg}$ three times a week could be used to minimise side-effect risk with subsequent titration according to clinical response. (Conditional)

- When using macrolides to reduce exacerbation rates, therapy should be offered for a minimum of 6 months. (Strong)

- Macrolides can be considered with the aim of improving QOL but may require a long period of therapy (eg, 1 year) for significant effects. (Conditional)

\section{Good practice points}

$\checkmark$ Therapies should be optimised in accordance with BTS Bronchiectasis Guidelines before considering long-term macrolide therapy (eg, airway clearance techniques and attendance at pulmonary rehabilitation courses).

$\checkmark$ Macrolides should only be started following discussion and shared decision-making between the patient and a respiratory specialist.

$\checkmark$ For safety purposes, an ECG should be performed prior to initiation of macrolide therapy to assess QTc interval. If QTc is $>450 \mathrm{~ms}$ for men and $>470 \mathrm{~ms}$ for women, this is considered a contraindication to initiating macrolide therapy. Baseline liver function tests should also be measured.

$\checkmark$ Patients should be counselled about potential adverse effects before starting therapy including gastrointestinal upset, hearing and balance disturbance, cardiac effects and microbiological resistance. Microbiological assessment of sputum should be performed before therapy, including investigation for NTM. Macrolide monotherapy should be avoided if an NTM is identified. When evaluating for NTM infection, macrolides should not be used for 2 weeks before microbiological testing.

$\checkmark$ Accurate assessment of baseline exacerbation rate should be determined before starting long-term macrolides for bronchiectasis.

$\checkmark \quad$ Liver function tests should be checked 1 month after starting treatment and then every 6 months. An ECG should be performed 1 month after starting treatment to check for new QTc prolongation. If present, treatment should be stopped.

$\checkmark$ Subsequent follow-up at 6 months and 12 months should determine whether benefit is being derived from therapy. If there is no benefit, treatment should be stopped.

$\checkmark$ Even if benefit is seen, consideration should be given to stopping treatment for a period each year, for example, over the summer. Such a drug holiday may help with reducing the development of resistance while maintaining efficacy because the vicious cycle has been broken.

See quick reference guide in online supplementary file 1.

\section{Research recommendations}

Long-term studies of microbiological impact of prolonged macrolide therapy.

Head-to-head comparison of different dose regimens for the same macrolide (eg, $250 \mathrm{mg}$ three times a week of azithromycin vs $500 \mathrm{mg}$ three times a week).

Head-to-head comparisons of different macrolides (eg, azithromycin vs erythromycin).

Prolonged studies of benefit and risk beyond 12 months of use.

Studies assessing the effect of macrolides following reintroduction of therapy after a break.

Use of bronchiectasis specific QOL measures in macrolide trials.

Comparison of outcomes in patients with different baseline exacerbation rates when treated with macrolides.

Comparison of outcomes in patients with different baseline microbiological culture/microbiome profiles when treated with macrolides.

Comparison of outcomes in patients with different baseline QOL scores when treated with macrolides.

Comparison studies of long-term macrolides to other oral or inhaled prophylactic regimens.

Studies looking into the benefits of combined macrolide and inhaled antibiotic regimens.

\section{SECTION 6: COPD \\ Introduction}

COPD is a progressive, inflammatory disease of the airways, characterised by ongoing development of non-reversible airflow limitation and acute episodes of exacerbation. Acute exacerbations are described as episodes of acute worsening of respiratory symptoms that necessitate a change in regular medication. ${ }^{76}$ The natural disease course, health-related QOL, hospital admissions and mortality associated with COPD are influenced by acute exacerbations. $^{77}$ In addition, acute exacerbations of COPD (AECOPD) are estimated to account for between 50\% and 75\% of the total costs for COPD treatment. ${ }^{78}$ Treatment interventions effective in preventing acute exacerbations are therefore of significant clinical benefit.

The mechanisms for COPD disease progression are complex and involve inflammatory and immune responses. Neutrophils, IL-17 and IL-23 are considered to play an active role in disease progression while CD8-positive T cells, neutrophils and macrophages mediate chronic inflammatory responses. ${ }^{79}$ Acute exacerbations of COPD are postulated to be caused by bacteria or bacteria in combination with viral infection in up to $50 \%$ of cases. ${ }^{80}$ Macrolide antibiotics possess both anti-inflammatory and antibacterial effects and are therefore an important potential treatment strategy to prevent AECOPD.

The potential mechanisms of action and clinical implication of long-term macrolide antibiotic therapy in COPD have been described and reviewed in several studies. ${ }^{81}$ Table 7 summarises the potential effects of macrolides on disease modulators in COPD.

\section{Evidence base}

To confirm the hypothesis that macrolide antibiotics are an effective treatment strategy in COPD to prevent acute exacerbations and possibly modify disease progression, several studies have been conducted yielding varied results. The evidence base for effectiveness of macrolide antibiotics in this context is compromised by the significant heterogeneity in populations 


\begin{tabular}{|c|c|}
\hline Macrolide action & Proposed mechanism of action in COPD \\
\hline Antimicrobial & $\begin{array}{l}\text { Direct antimicrobial action against bacteria commonly } \\
\text { associated with exacerbations in COPD (Haemophilus } \\
\text { influenzae, Moraxella catarrhalis, Streptococcus } \\
\text { pneumoniae, Chlamydia pneumoniae and Mycoplasma } \\
\text { pneumoniae) } \\
\text { Indirect antimicrobial action through inhibition of bacterial } \\
\text { quorum sensing }\end{array}$ \\
\hline $\begin{array}{l}\text { Reduce airway } \\
\text { inflammation }\end{array}$ & $\begin{array}{l}\text { Reduce adhesion molecule expression by epithelial and } \\
\text { endothelial cells (ICAM-1, V-CAM-1) } \\
\text { Modulates pro-inflammatory cytokine release (IL-1, IL-6, } \\
\text { IL-8, TNF } \propto \text { ) } \\
\text { Inhibits signalling pathways (AP-1 and NK-kB transcription } \\
\text { factors) } \\
\text { Influences Toll-like receptor expression and activity }\end{array}$ \\
\hline Mucus production & $\begin{array}{l}\text { Antibacterial action inhibits bacterial induced MUC5AC } \\
\text { production } \\
\text { Downregulates protein secretion and expression of } \\
\text { MUC5AC mRNA } \\
\text { - Inhibits chloride secretion }\end{array}$ \\
\hline Innate immunity & $\begin{array}{l}\text { Macrophages: enhance phagocytosis and monocyte- } \\
\text { macrophage differentiation and reduces metalloproteinase } \\
\text { production } \\
\text { Neutrophils: reduce chemotactic response, elastolytic } \\
\text { activity and tissue accumulation. Inhibits superoxide anion } \\
\text { production. Enhances superoxide dismutase activity }\end{array}$ \\
\hline Adaptive immunity & $\begin{array}{l}\text { Lymphocytes: inhibit pro-inflammatory cytokine production } \\
\text { and increases apoptosis } \\
\text { Dendritic cells: modulate pro-inflammatory cytokine } \\
\text { production }\end{array}$ \\
\hline $\begin{array}{l}\text { Prokinetic in GI } \\
\text { tract }\end{array}$ & $\begin{array}{l}\text { Reduce gastro-oesophageal reflux and microaspiration } \\
\text { leading to airway inflammation }\end{array}$ \\
\hline
\end{tabular}

investigated, intervention (type, dose and duration of macrolide), definition of outcomes measured and lack of phenotyping COPD to identify subgroups most likely to respond.

Six meta-analyses were identified in addition to the published ERS/ATS Guideline on Prevention of COPD exacerbations. ${ }^{82}$ Nine RCTs were reviewed and included in the guideline. The COLUMBUS trial published in $2014^{83}$ and the small RCT by Simpson et $a l^{84}$ were included in data analysis of one of the meta-analysis and the ERS/ATS guideline. ${ }^{82}$ Table 8 illustrates the matrix of evidence reviewed, all RCTs and meta-analyses published since 2000 with evidence included in meta-analysis.

\section{Randomised trials excluded}

The Cochrane review assessing prophylactic antibiotic therapy for COPD included in the pooled analysis the RCTs performed by Sethi and colleagues ${ }^{85}$ in 2010 (pulsed moxifloxacin $400 \mathrm{mg}$ once a day for 5 days every 8 weeks) and Mygind and colleagues ${ }^{86}$ in 2010 (pulsed azithromycin $500 \mathrm{mg} 3$ days a month for 36 months). The scope of this guideline does not include pulsed doses of quinolone antibiotics, and this study was not included in the data synthesis. Mygind's (2010) unpublished conference abstract with limited access to data on randomisation, allocation and incomplete outcome data could not be included in analysis of the evidence due to the risk of bias. Shafuddin and colleagues published a three-armed RCT in 2015, comparing usual care, roxithromycin and roxithromycin/doxycycline combination. Roxithromycin is not available in the UK, and this therefore prevents generalisability of the evidence, so on this basis, the study was excluded. ${ }^{87}$

\section{Cohort studies}

Several retrospective cohort studies were identified, with the largest two (Pomares et al and Yamaya et al) including a total number of 326 patients. ${ }^{88} 89$ The quality of evidence in reporting exacerbations and QOL was deemed poor due to the risk of selection and attrition bias.

\section{Included studies}

Based on the aforementioned appraisal of evidence, the included studies for recommendations in this guideline were focused on RCTs and systematic reviews as demonstrated in the evidence matrix (table 8).

The RCTs demonstrated significant heterogeneity (in terms of intervention, population and outcomes), and therefore recommendations are limited by uncertainty in the evidence base for a specified intervention. Table 9 demonstrates the trials with variation in detail.

\section{Other recent guidance}

\section{GOLD 2019}

In patients with COPD optimised with inhaled therapy who are still experiencing exacerbations, the best available evidence exists for the use of azithromycin, especially in those who are not current smokers. Consideration to the development of resistant organisms should be factored into decision-making. ${ }^{90}$

\section{NICE 2018}

Recommends after non-pharmacological and pharmacological optimisation to consider azithromycin (usually $250 \mathrm{mg}$ three times a week) for people with COPD more prone to daily sputum production if they have stopped smoking and have more than four exacerbations per year, prolonged exacerbations or exacerbations resulting in hospital admission.

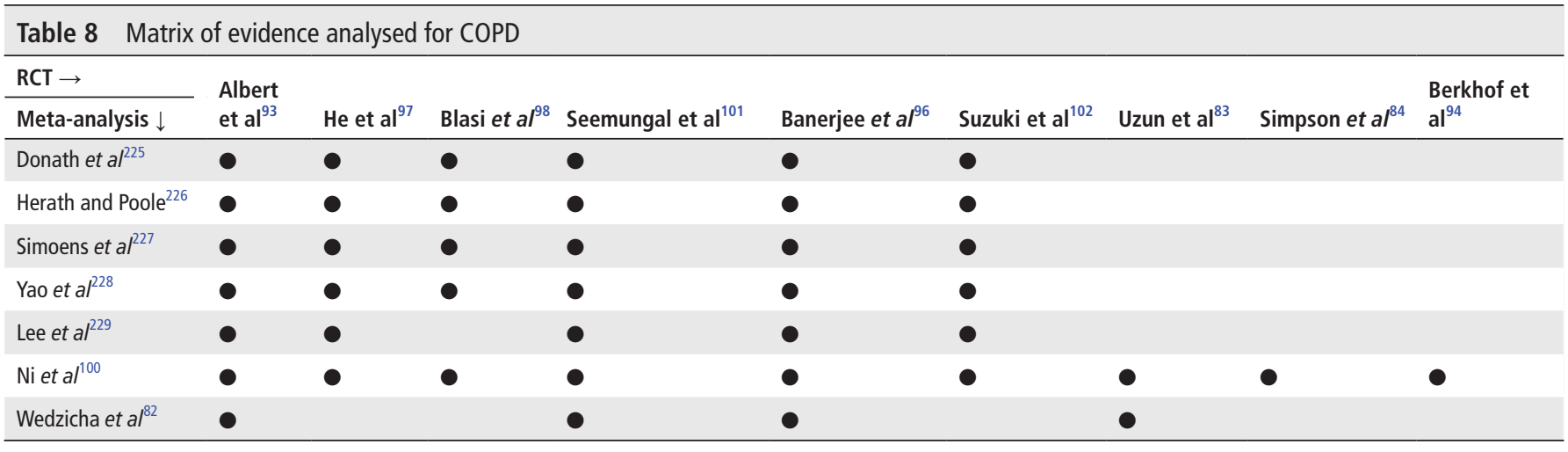


Table 9 Summary of COPD trials

\begin{tabular}{|c|c|c|c|c|c|c|}
\hline & $\begin{array}{l}\text { Country } \\
\text { conducted }\end{array}$ & $\begin{array}{l}\text { Population } \\
\text { Mean age: intervention/control } \\
\text { COPD intervention/control } \\
\text { Required an exacerbation in year before }\end{array}$ & Intervention & $\begin{array}{l}\text { Follow-up on } \\
\text { treatment }\end{array}$ & $\begin{array}{l}\text { Patients } \\
\text { Intervention/ } \\
\text { control }\end{array}$ & Primary outcome \\
\hline Albert et al $l^{93}$ & USA & $\begin{array}{l}\text { Mean age: } 65 / 66 \\
\text { COPD FEV } \%: 39 / 40 \\
\text { Yes-at least one exacerbation }\end{array}$ & Azithromycin $250 \mathrm{mg}$ a day & 12 months & $558 / 559$ & Time to first exacerbation \\
\hline He et $a l^{97}$ & China & $\begin{array}{l}\text { Mean age: } 68.8 / 69.3 \\
\text { COPD } \mathrm{FEV}_{1} \%: 44.3 / 42.1 \\
\text { No }\end{array}$ & $\begin{array}{l}\text { Erythromycin } 150 \mathrm{mg} \text { three } \\
\text { times a day }\end{array}$ & 6 months & $18 / 18$ & $\begin{array}{l}\text { Exacerbations airway } \\
\text { inflammation }\end{array}$ \\
\hline $\begin{array}{l}\text { Seemungal et } \\
a l^{230}\end{array}$ & UK & $\begin{array}{l}\text { Mean age: } 66.5 / 67.8 \\
\text { COPD } \mathrm{FEV}_{1} \%: 49.3 / 50.6 \\
\text { No }\end{array}$ & $\begin{array}{l}\text { Erythromycin (stearate tablets) } \\
250 \mathrm{mg} \text { bd }\end{array}$ & 12 months & $53 / 56$ & $\begin{array}{l}\text { Exacerbations airway } \\
\text { inflammation }\end{array}$ \\
\hline Suzuki et al $\left.\right|^{102}$ & Japan & $\begin{array}{l}\text { Mean age: } 69.1 / 71.7 \\
\text { COPD FEV } 1 \text { L/s: } 1.47 / 1.30 \\
\text { No }\end{array}$ & $\begin{array}{l}\text { Erythromycin (ethylsuccinate } \\
\text { tablets) } 200-400 \mathrm{mg} \text { a day }\end{array}$ & 12 months & $55 / 54$ & $\begin{array}{l}\text { Frequency of common cold } \\
\text { and exacerbations }\end{array}$ \\
\hline Uzun et $a l^{83}$ & Netherlands & $\begin{array}{l}\text { Mean age: } 64.7 / 64.9 \\
\text { COPD FEV } \%: 44.2 / 45 \\
\text { Yes-three or more exacerbations }\end{array}$ & $\begin{array}{l}\text { Azithromycin } 500 \mathrm{mg} \text { three } \\
\text { times a week }\end{array}$ & 12 months & $47 / 45$ & Rate of exacerbations \\
\hline
\end{tabular}

\section{ERS/ATS recommendation}

Patients with COPD with moderate to very severe airflow obstruction (post-bronchodilator $\mathrm{FEV}_{1} / \mathrm{FVC}<0.70$ and an $\mathrm{FEV}_{1} \%$ predicted of $<80 \%$ ) and exacerbations despite optimal inhaled therapy, treatment with a macrolide antibiotic to prevent future exacerbations is suggested as a conditional recommendation. ${ }^{9}$

\section{Cochrane systematic reviews}

Prophylactic antibiotics for patients with COPD that included macrolide therapy found for every eight participants treated, one person would be prevented from suffering an exacerbation. Not all the antibiotic regimens had the same impact on exacerbations, with results suggesting that antibioticsPrescribing off-label given at least three times per week may be more effective than pulsed antibiotic regimens. ${ }^{10}$

\section{Symptoms and QOL}

Health status measurement is a standardised and objective means of quantifying the impact of disease on patients' daily life, health and well-being. Health status questionnaires usually address emotional and psychological effects of the illness as well as the physical. The St George's Respiratory Questionnaire (SGRQ) is validated to measure health impairment in patients with COPD. It is in two parts; Part 1 produces the Symptoms score, and Part 2 the Activity and Impacts scores, which results in a total score..$^{91} 92$

Macrolide therapy (azithromycin) improved healthrelated QOL in four studies (1323 patients) which used the SGRQ. ${ }^{83} 849394$ There was significant heterogeneity among the studies $\left(\mathrm{I}^{2}=97.10 \%\right)$.

The Berkhof et al study ${ }^{94}$ assessed QOL in patients with COPD, chronic cough and at least one AECOPD in the previous year. Patients with COPD and chronic cough is a heterogeneous group of patients, but from this single study, patients with more severe cough reported better QOL (LCQ) on macrolide treatment. Three other studies did not include LCQ in the diseasespecific impact on QOL and the impact of cough could therefore not be pooled and analysed for these studies. ${ }^{83} 8493$ The pooled assessment for long-term azithromycin suggests its use improved QOL as measured by the SGRQ total score (MD $=-2.12,95 \%$ CI -3.44 to $\left.-0.79, \mathrm{p}=0.002, \mathrm{I}^{2}=0 \%\right)$. Although statistically significant, the results did not meet clinical significant outcome of 4 units as minimum clinically important difference (MCID)..$^{95}$

Pooled analysis for the Albert et al 2011 and Uzun et al 2014 studies on QOL (SGRQ) in 491 patients on azithromycin and 498 patients on placebo demonstrated improved total scores (MD 2.18 lower, 95\% CI 1.53 lower to 2.82 lower), and in symptoms (MD 3.36 lower, 95\% CI 2.42 lower to 4.29 lower), activity (MD 1.82 lower, 95\% CI 1.03 lower to 2.62 lower) and impacts (mean difference 2.04 lower, 95\% CI 1.28 lower to 2.81 lower). ${ }^{8393}$ The Albert study had a significant 12 -month dropout rate in both intervention (444 completed SGRQ at 12 months of 556 enrolled) and control (453 completed SGRQ at 12 months of 555 enrolled patients) groups, and this may have affected the results. $^{93}$

Banerjee reported improved health status (SGRQ) at 12 weeks on clarithromycin measured in total, symptom, and impact scores. ${ }^{96}$ Erythromycin $125 \mathrm{mg}$ once daily for 6 months did not demonstrate significant changes in quality of life (SGRQ) at 3 and 6 months. ${ }^{97}$

\section{Exacerbations}

Nine RCTs were identified that reported the effect of long-term macrolide antibiotics on AECOPD. The study entry criteria varied in terms of the number of exacerbations in patients in the year prior to the trial, the severity of COPD and the definition of the severity of an acute exacerbation.

\section{Variations}

The COLUMBUS trial ${ }^{83}$ enrolled patients with at least three exacerbations in the year prior to entering the trial, Blasi et $a l^{98}$ reported the number of exacerbations in patients the year before entering the trial as $3.1( \pm 0.9)$ and $3.0( \pm 1.1)$ in the control and intervention arm, respectively. The largest RCT (Albert et al 2011) included in the entry criteria at least one acute exacerbation in the year prior to trial entry. ${ }^{93}$ The remainder of studies did not define number of exacerbations prior to entering the trial in the inclusion criteria.

There was significant variation in severity of disease of patients enrolled in trials, including reporting of severity of disease and airflow limitation measurements reported as the 
mean $\mathrm{FEV}_{1}$ measured in litres or as percentage predicted. Albert et al described severity (GOLD criteria ${ }^{99}$ ) of patients in the intervention/control arm as follows: GOLD $2(26 \% / 26 \%)$, GOLD $3(40 \% / 40 \%)$ and GOLD $4(34 \% / 33 \%) \cdot{ }^{93}$ Uzun et al included patients as follows: GOLD 1 (4/7\%), GOLD 2 (30/27\%), GOLD $3(38 / 40 \%)$ and GOLD $4(28 / 22 \%) .{ }^{83}$

The use of concomitant medication to treat COPD varied in the trials and appears to correlate with published guidelines at the time of trial recruitment (eg, NICE and GOLD guidelines). ${ }^{99} 100$ Treatment recommended during the studies included sustained release theophylline, inhaled corticosteroids, inhaled anticholinergic and beta adrenergic agents.

The studies included in the data synthesis used 14-membered and 15 -membered-ring compound macrolide antibiotics. Five studies used azithromycin in doses that varied from $750 \mathrm{mg}$ to $1750 \mathrm{mg}$ a week, ${ }^{83} 84939498$ erythromycin in two studies (1400$3500 \mathrm{mg}$ per week $)^{97101}$ and one study used clarithromycin 3500 mg per week. ${ }^{96}$

\section{Exacerbation rate and number of exacerbations}

Seven randomised control studies involving 1614 patients reported the number of acute exacerbations during the period

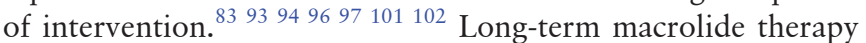
was shown in a meta-analysis to significantly reduce acute exacerbations in the intervention group when compared with the comparison group ( $\mathrm{RR}=0.7,95 \% \mathrm{CI} 0.56$ to $0.87, \mathrm{p}<0.01$, $\left.\mathrm{I}^{2}=66.43 \%\right) .{ }^{100}$ The Suzuki et al 2001 trial was non-blinded and in appraisal of the study methodology, this raised concerns for bias. ${ }^{102}$ Analysis of the remaining six studies continued to show benefit for macrolide therapy over placebo, with a $20 \%$ relative risk reduction of AECOPD ( $\mathrm{RR}=0.8,95 \% \mathrm{CI} 0.72$ to 0.88 , $\mathrm{p}<0.01)$.

Eight RCTs reported the rate of exacerbation per patient per year in a total of 1582 patients. ${ }^{83} 849396-98101102$ The long-term macrolide group had a significant reduction in the rate of exacerbations ( $\mathrm{RR}=0.58,95 \% \mathrm{CI} 0.43$ to $0.78, \mathrm{p}<0.01, \mathrm{I}^{2}=67.8 \%$ ). The recent published ERS/ATS guideline pooled data for studies that included treatment for 12 months and excluded the Suzuki trial $^{102}$ (due to risk of bias, non-blinded study as described above) and demonstrated a reduced rate of AECOPD ( $R R=0.76,95 \%$ CI 0.68 to 0.86$)^{82}$

Data analysis demonstrated no significant difference in exacerbation rates at 3 months; it is recognised that these studies were likely underpowered to demonstrate effect size. Banerjee et al reported the effects of clarithromycin for 3 months in 67 patients $(\mathrm{RR}=3.27,95 \% \text { CI } 0.53 \text { to 20.18, } \mathrm{p}=0.2)^{96}$ and Simpson et al on 30 patients with azithromycin for 12 weeks $(\mathrm{RR}=0.38,95 \%$ CI 0.14 to $1.05, \mathrm{p}=0.06) .{ }^{84}$ The number of exacerbations at 3 months of treatment did not demonstrate significant variation, as reported by Berkhof et al, with azithromycin in 84 patients $(\mathrm{RR}=0.46,95 \% \mathrm{CI} 0.18$ to $1.18, \mathrm{p}=0.11) .{ }^{94}$ In contrast, studies reporting exacerbation rates at 6-12 months ${ }^{83} 9398$ in pooled assessment with azithromycin (1231 patients) demonstrated significant reduction $(\mathrm{RR}=0.59,95 \%$ CI 0.37 to $0.93, \mathrm{p}=0.02)$ and with erythromycin (254 patients) for 6-12 months ${ }^{97101} 102 \mathrm{a}$ similar effect $(\mathrm{RR}=0.53,95 \%$ CI 0.43 to $0.83, \mathrm{p}=0.01)$.

\section{Onset of first exacerbation}

Macrolide therapy significantly delayed onset of first exacerbation. Pooled data of patients on macrolide antibiotics for 12 months demonstrates a mean 81-day delay in first AECOPD (95\% CI 53.3 to 109.8 days longer) ${ }^{82}$ and the study by Seemungal et al in 109 patients with erythromycin for 12 months demonstrated a mean 182 days delay in first AECOPD $(p=0.02){ }^{101}$

Daily use of azithromycin long term (12 months) was demonstrated in Albert et al (1117 patients) to significantly reduce exacerbation rate $(\mathrm{RR}=0.83,95 \% \mathrm{CI} 0.72$ to $0.95, \mathrm{p}=0.01) .^{93}$ Two studies adopted intermittent dosing regimens (see table 9, Uzun et $\mathrm{l}^{83}$ and Blasi et $a l^{98}$ ) for azithromycin over 6-12 months involving 114 patients demonstrated reduced exacerbation rates $(\mathrm{RR}=0.41,95 \% \mathrm{CI} 0.18$ to $0.96, \mathrm{p}=0.04) .{ }^{83}{ }^{98}$ Using the intermittent dosing regimen with azithromycin over 12 months rather than 6 months, as reported by Uzun et al, appeared to be more effective to reduce exacerbation rate $(\mathrm{RR}=0.58,95 \% \mathrm{CI}$ 0.42 to $0.80, \mathrm{p}<0.01){ }^{83}$

\section{Subgroups}

Han et al published subgroup analysis of the largest RCT (1117 patients) using azithromycin $250 \mathrm{mg}$ a day for 12 months. ${ }^{103}$ There was no convincing evidence that treatment efficacy was affected by sex, presence of chronic bronchitis, use of oxygen therapy or concomitant COPD therapy. Long-term azithromycin therapy was found to be more effective in older patients $(>65$ years, relative hazard $0.59,95 \% \mathrm{CI} 0.57$ to $0.74, \mathrm{p}<0.01$ ) and in patients who stopped smoking (ex-smoker relative hazard 0.65 , $95 \%$ CI 0.55 to 0.77 ; smokers relative hazard $0.99,95 \%$ CI 0.71 to $1.38 ; \mathrm{p}$ value for interaction $=0.03$ )

\section{Severity of exacerbations on macrolide therapy}

The impact of long-term macrolide therapy on the severity of exacerbations was not reported in many studies. Seemungal et al showed that macrolide use was associated with a lower median number of exacerbation days. ${ }^{101}$ The macrolide group reported median exacerbation days of 9 with IQR 6-13 days, and the placebo group had median of 13 exacerbation days with IQR 6-24 days $(p=0.036) .{ }^{101}$ There is insufficient evidence to conclude what the impact on severity of AECOPD is in patients on long-term macrolide therapy.

\section{Hospitalisation for AECOPD}

Five studies involving 1424 patients reported on the number of hospitalisations due to acute exacerbations of COPD as secondary outcomes. ${ }^{83} 939498101$ Pooled data, as reported by Ni et al, did not demonstrate a significant reduction in hospital admissions in patients with AECOPD on macrolide therapy in comparison with the control group (RR $0.89,95 \%$ CI 0.64 to $1.24, p=0.50$ ). ${ }^{100}$ Albert $e$ al found a non-significant trend towards reduction in the rate of hospital admissions using macrolides,${ }^{93}$ whereas Uzun and colleagues found no significant delay in time to first hospitalisation in patients on long-term macrolide therapy. ${ }^{83}$

The published evidence does not support the use of long-term macrolides in patients with COPD to reduce severity of exacerbations or requirement for hospitalisation due to exacerbation. The studies were underpowered and definition of severity of exacerbations not comparable with pool data.

\section{Lung function tests and disease progression}

The natural history of COPD is characterised by progressive airflow limitation and historically, $\mathrm{FEV}_{1}$ decline has been considered the single most important marker of disease progression. ${ }^{104}$ However, data obtained in two different retrospective cohorts have shown that $\mathrm{FEV}_{1}$ decline is not invariably progressive and FEV is only weakly correlated with patient-related outcomes. ${ }^{105} 106$ 
Randomised trials assessing the impact of long-term macrolide treatment in COPD have not reported on disease progression as a secondary outcome. Rate of decline in $\mathrm{FEV}_{1}$ as single measure is not accurate in the measurement of disease progression, or in assessing the effect of therapeutic interventions in altering the progression of the disease process. ${ }^{107}$

Lung function was reported in four studies. Pooled analysis was not possible due to the varied ways in which results were reported. Banerjee et al reported no difference in the intervention and placebo groups after 12 weeks of clarithromycin in spirometry measurements or shuttle walk distance. ${ }^{96}$ Simpson et al reported no difference in $\mathrm{FEV}_{1}$ reported as percentage predicted with 12 weeks of azithromycin $250 \mathrm{mg}$ a day (30 patients, 15 in each arm). ${ }^{84} \mathrm{No}$ difference in $\mathrm{FEV}_{1}$ after 12 months' treatment with erythromycinstudy was withdrawn due to $250 \mathrm{mg}$ or placebo was demonstrated by Seemungal and colleagues in patients with COPD. ${ }^{101}$ No significant changes were shown between groups in post-bronchodilator $\mathrm{FVC}, \mathrm{FEV}_{1}$ or 6 min walk test in patients on azithromycin $500 \mathrm{mg}$ three times a week after 12 months ${ }^{83}$

There is no evidence that long-term macrolide therapy results in improvements, or lessened decline, of lung function parameters in patients with COPD.

\section{Safety and adverse events}

Long-term treatment with macrolides has been associated with several adverse effects. ${ }^{108}$ The most common side effects relate to the gastrointestinal tract by stimulating gastrointestinal motility through motilin-like activity. Symptoms including anorexia, nausea, vomiting, diarrhoea and abdominal pain were reported in patients with COPD in the studies reviewed. Cessation of treatment due to gastrointestinal side effects were reported in the studies published by Banerjee et al, Suzuki et al, Seemungal et al, He et al and Blasi et al. ${ }^{96-98} 101102$ Uzun published sideeffect profiles, indicating that diarrhoea was the only event that was higher statistically significantly more frequent in the azithromycin group compared with placebo. ${ }^{83}$

Albert et al reported hearing impairment in 142 (25\%) patients with COPD treated with azithromycin $250 \mathrm{mg}$ a day and in $110(20 \%)$ patients receiving placebo $(\mathrm{p}=0.04) .{ }^{93}$ A small, but significant difference was seen at 3-month review in audiology measurements (in all four sound frequencies) in the treatment arm. $^{93}$ Repeat audiology testing demonstrated improvement regardless if azithromycin was stopped or not. The authors postulate too stringent criteria for measurable deficit and over reporting of hearing impairment as explanation. None of the other studies reported on hearing impairment as an outcome.

Erythromycin and clarithromycin are both associated with ototoxicity that includes vertigo, hearing loss, deafness and tinnitus. ${ }^{109}$ A single patient receiving long-term erythromycin in the Seemungal et al study was withdrawn due to significant tinnitus. ${ }^{101}$

The recognised side-effect profile associated with macrolide therapy includes allergic reactions, skin eruptions, hepatotoxicity, cardiac arrhythmias and QTc prolongation. ${ }^{110-112}$ These side effects were not reported as adverse events in the studies reviewed based on the trial protocols and exclusion criteria (designed to exclude high-risk patients specifically with regards to cardiac toxicity). Three studies including 212 patients reported four cardiovascular events in the treatment arm and two in the control arm $(p=0.43){ }^{839497}$ Albert and colleagues reported the death rate due to cardiovascular events in both the azithromycin and placebo arm as $0.2 \%$.

Pooled analysis of all nine RCTs reviewed demonstrated higher adverse events reported in the intervention group, with gastrointestinal side effects the most commonly reported (OR $1.55,95 \%$ CI 1.00 to $2.39, \mathrm{p}=0.05)^{84939496-98101102}$ Albert and colleagues reported only $11 / 558$ patients in the treatment arm on azithromycin that had to stop the treatment due to GI side effects and 6/559 patients in the control group stopped placebo for the same reason. ${ }^{93}$

\section{Antimicrobial resistance}

Macrolide antibiotics have direct antibacterial activity against Gram-positive, Gram-negative and atypical bacteria such as Legionella spp, Mycoplasma and Chlamydia spp. Macrolides inhibit RNA-dependent protein synthesis by reversibly binding to the P-site on the 50S subunit of bacterial ribosomes and inhibit transpeptidation or translocation of nascent peptides. ${ }^{81}$

The severity and rate of AECOPD have been linked to both $C$. pneumoniae and Haemophilus influenzae. ${ }^{113-117}$ The antimicrobial activity of macrolide antibiotics with possible reduction in bacterial load may explain in part the effect on exacerbation rate seen in clinical trials in patients with COPD. The acquisition of macrolide resistance with long-term low-dose use of macrolide antibiotics remain uncertain in patients with COPD.

Seven studies monitored and reported on macrolide antibiotic susceptibility changes during treatment period. ${ }^{83939496-98101}$

Albert et al found only 15\% of patients could expectorate sputum after a 3 -month period on macrolide therapy. ${ }^{93}$ Investigators used sputum in patients who could expectorate and nasopharyngeal swabs to report on colonisation and resistance. This study subsequently reported Staphylococcus aureus as the most common organism isolated (treatments group 60 (10.7\%) vs placebo group $71(12.7 \%)$ ) likely due to nasopharyngeal sampling. Moraxella spp and S. pneumoniae were both isolated and did not show significant difference between treatment and placebo arms in the study. During the 12-month study period, significantly more patients in the placebo group became colonised (172 patients) versus 66 patients in the treatment arm on azithromycin $250 \mathrm{mg}$ a day $(\mathrm{p}<0.001)$. Newly colonised patients in the treatment group demonstrated significantly higher resistance to macrolide antibiotics $81 \%$ compared with $41 \%$ in the placebo group $(\mathrm{p}<0.001)$.

Seemungal et al reported in 109 patients receiving erythromycin $250 \mathrm{mg}$ twice a day over a 12-month period that only one patient developed resistance to $S$. pneumoniae. H. influenzae was isolated in 22 of the 109 patients; all of the isolates were found to be resistant to erythromycin. ${ }^{101}$

At baseline, Uzun et al obtained 22 sputum samples in the treatment group and 20 sputum samples in the placebo group. After a 12-month study period, 51 sputum samples in the treatment group and 57 sputum samples in the placebo group were analysed. This study also reported fewer new colonised patients in the treatment arm, 4 patients versus 12 patients $(\mathrm{p}=0.044)$. Macrolide resistance was reported in $6 \%$ (3 patients) receiving azithromycin $500 \mathrm{mg}$ three times a week and in 24\% (11 patients) receiving placebo for the 12-month period $(\mathrm{p}=0.036){ }^{83}$

Banerjee et al and He et al did not observe significant differences in colonisation rate or resistance to macrolide antibiotics, but these studies were shorter in duration, 3 and 6 months, respectively. ${ }^{96} 97$

In a single-centre, single-blind randomised placebo controlled trial, Brill et al demonstrated, after 3 months of azithromycin $250 \mathrm{mg}$ three times a week, no difference in airway inflammation or bacterial load $\left(0.42 \log _{10} \mathrm{cfu} / \mathrm{mL}\right)$ in sputum when compared with placebo $(0.08 \mathrm{CI} 0.38$ to 0.54$) .{ }^{118}$ 


\section{Mortality}

In studies where mortality was specifically reported, no significant difference was observed between the control and macrolide groups $(\mathrm{RR}=0.9,95 \% \mathrm{CI} 0.48$ to 1.69$) .{ }^{8393102}$

It should be noted that the included studies all report mortality as secondary outcome measure, and longer-term potential impact on mortality (through for example reduced exacerbations) may not manifest in the 12-month follow-up period which was typical for these studies. ${ }^{106119120}$ There is therefore insufficient evidence to indicate a reduction in mortality with the use of long-term macrolides, but no evidence of increased mortality in relation to treatment.

\section{Outcome weighting}

The guideline group identified rate of AECOPD, time to first exacerbation, hospitalisation, serious adverse events and mortality as critical outcomes. Important outcomes identified include QOL, disease progression, lung function tests, airway inflammation and acquisition of macrolide resistance.

\section{Evidence summary}

Long-term macrolide treatment showed statistically significant improvement in QOL in patients with COPD as measured by SGRQ, but this does not reach the MCID-4 unit change. (Moderate)

Long-term macrolide antibiotics are effective in reducing the acute exacerbation rate in patients with COPD with high exacerbation rates (ie, more than three exacerbations per year, prolonged exacerbations or exacerbations resulting in hospitalisation). (Moderate)

Treatment courses of 12 months demonstrated the biggest effect size in reduction of exacerbation rate. (Low)

The number of hospitalisations was not significantly reduced in patients receiving long-term macrolide therapy. (Moderate)

No significant mortality benefit has been shown in 12-month follow-up studies using macrolide therapy, and there is insufficient evidence to demonstrate the effect of long-term macrolide therapy on the mortality in patients with COPD. (Low)

There is no evidence to suggest that long-term macrolide therapy impacts on disease progression or spirometry measurements and exercise capacity measurements. (Low)

Insufficient evidence is available on airway colonisation and acquisition of macrolide resistance in patients with COPD receiving long-term, low-dose macrolide therapy. (Moderate)

\section{Recommendations}

- Long-term macrolide therapy could be considered for patients with COPD with more than three acute exacerbations requiring steroid therapy and at least one exacerbation requiring hospital admission per year to reduce exacerbation rate. (Conditional)

- Long-term macrolide therapy could be considered for a minimum of 6 months and up to 12 months to assess the impact on exacerbation rate. (Conditional)

\section{Good practice points}

$\checkmark \quad$ Non-pharmacological and pharmacological therapies should be optimised prior to considering long-term macrolide therapy. This includes smoking cessation, optimised inhaler technique, optimised self-management care plan, airway clearance techniques and attendance at pulmonary rehabilitation courses. $\checkmark$ Macrolides should only be started following discussion and shared decision-making between the patient and a respiratory specialist.

$\checkmark \quad$ For safety purposes, an ECG should be performed prior to initiation of macrolide therapy to assess QTc interval. If QTc is $>450 \mathrm{~ms}$ for men and $>470 \mathrm{~ms}$ for women, this is considered a contraindication to initiating macrolide therapy. Baseline liver function tests should also be measured.

$\checkmark \quad$ Patients should be counselled about potential adverse effects before starting therapy including gastrointestinal upset, hearing and balance disturbance, cardiac effects and microbiological resistance.

$\checkmark$ Microbiological assessment of sputum should be performed before therapy, including investigation for NTM. Macrolide monotherapy should be avoided if an NTM is identified. Repeat assessments are recommended with clinical decline or during exacerbations to monitor resistance patterns.

$\checkmark$ Accurate assessment of baseline exacerbation rate should be determined before starting long-term macrolides for patients with COPD and a CT scan should be considered to exclude a possible diagnosis of bronchiectasis.

$\checkmark$ A risk:benefit profile should be considered in each individual if significant side effects from oral macrolide therapy develop. If gastrointestinal side effects occur at the higher dose of azithromycin (500 mg thrice weekly), a dose reduction to azithromycin $250 \mathrm{mg}$ thrice weekly could be considered if macrolide therapy has been of clinical benefit.

$\checkmark \quad$ Liver function tests should be checked 1 month after starting treatment and then every 6 months. An ECG should be performed 1 month after starting treatment to check for new QTc prolongation. If present, treatment should be stopped.

$\checkmark$ Subsequent follow-up at 6 and 12 months should determine whether benefit is being derived from therapy by using objective measures such as the exacerbation rate, CAT score or QOL as measured by a validated assessment tool such as SGRQ. If there is no benefit, treatment should be stopped.

$\checkmark \quad$ It is not necessary to stop prophylactic azithromycin during an acute exacerbation of COPD unless another antibiotic with potential to affect the QT interval has also been prescribed.

See quick reference guide in online supplementary file 1.

\section{Research recommendations}

Long-term ( $>12$ months) follow-up trials to evaluate impact of long-term macrolide therapy on mortality, antimicrobial resistance, long-term potential cardiac toxicity and disease progression.

Studies to evaluate the impact of short-term breaks in chronic therapy with long-term macrolide antibiotics are needed. Studies phenotyping COPD in large trials where subgroup analysis can potentially identify groups of patients with COPD who will benefit most from long-term macrolide therapy.

Trials investigating head to head the benefits and adverse effects of oral agents that reduce acute exacerbations in patients with COPD (long-term, low-dose macrolides, carbocysteine, roflumilast).

\section{SECTION 7: BRONCHIOLITIS OBLITERANS (INCLUDING POST TRANSPLANTATION) \\ Introduction}

Bronchiolitis obliterans is a condition characterised by subepithelial inflammatory and fibrotic narrowing of small airways 
within the lung. It occurs following a variety of insults to the lung such as infections or inhaled toxins. It also occurs in the context of systemic diseases such as rheumatoid arthritis and other connective tissue diseases. It can occur as a complication of transplantation of lung or haematopoietic stem cells (HSCT). The histological correlate is obliterative bronchiolitis or constrictive bronchiolitis. ${ }^{121}$

In the context of lung transplantation, bronchiolitis obliterans causing lung function impairment without histological confirmation has been called bronchiolitis obliterans syndrome (BOS). A standard definition of BOS was first developed in $1993^{122}$ based on changes in $\mathrm{FEV}_{1}$ following transplantation. The definition has undergone subsequent revisions in $2002^{123}$ and in $2014^{124}$ when the over-arching term of chronic lung allograft dysfunction (CLAD) with subdivisions of restrictive CLAD (restrictive allograft syndrome, RAS) and obstructive CLAD (BOS) were proposed. BOS is common after lung transplantation occurring in over 50\% of those surviving more than 5 years. It is less common after HSCT, between $2 \%$ and $14 \%$ in the first 5 years post transplant. Long-term macrolides have been used both to prevent BOS and to treat BOS when it occurs. Several observational studies have noted a positive response to azithromycin in a proportion of patients with BOS following lung transplantation, especially those with neutrophilic bronchoalveolar lavage results. This has been labelled neutrophilic reversible allograph dysfunction or azithromycin responsive allograph dysfunction distinguishing it from the non-reversible CLAD.

\section{Evidence base}

There is a significant body of literature relating to bronchiolitis obliterans occurring in the context of lung or HSCT. In the lung transplantation field, the majority of published studies are case series. There is marked variation between studies in patient groups, concomitant immunosuppressive regimens, choice of macrolide and dosing regimens, and choice of outcome measures making meaningful systematic analysis of the data impossible. In patients with bronchiolitis obliterans complicating HSCT, there are fewer studies, predominantly case series, also with a high degree of variation in populations, background immunosuppressive regimens, macrolide interventions (often in conjunction with other novel therapeutic agents) and outcome measures precluding meaningful systematic analysis.

\section{Macrolides in BOS/CLAD following lung transplantation}

An International Society for Heart and Lung Transplantation/ American Thoracic Society (ATS)/European Respiratory Society (ERS) clinical practice guideline on the diagnosis and management of BOS was published in 2014 following the GRADE approach to develop specific clinical recommendations including the use of azithromycin. ${ }^{124}$ This guideline identified 10 studies (one observational study and nine case series) that described the effects of azithromycin on the lung function of lung transplant patients with BOS. It also identified two observational studies that described the effect of azithromycin on mortality in lung transplant patients with BOS. The guideline recommended a trial of azithromycin $(250 \mathrm{mg}$ orally daily for 5 days and then thrice weekly for a minimum of 3 months) for lung transplant recipients who developed a decline in $\mathrm{FEV}_{1}$ consistent with the onset of BOS. The recommendation was conditional.

There have been two RCTs investigating the role of macrolides in BOS following lung transplantation. Vos et al examined the effect of azithromycin versus placebo for the prevention of BOS following lung transplantation. ${ }^{125}$ Corris et al studied the effect of azithromycin versus placebo in the treatment of patients who developed BOS following lung transplantation. ${ }^{126}$ In the Vos et al single-centre paper, patients who had undergone lung transplantation were randomised to receive either azithromycin, $250 \mathrm{mg}$ three times a week (40 patients) or placebo (43 patients) for 2 years in a fully blinded study. Primary end points were freedom from BOS and survival at 2 years. If patients developed BOS on placebo, they were switched to open-label 'rescue' treatment with azithromycin. Patients receiving prophylactic azithromycin were less likely to develop BOS than those on placebo $(12.5 \%$ vs $44.2 \%, \mathrm{p}=0.0017)$. Overall survival was comparable between groups. ${ }^{125}$ This group of investigators have subsequently published a post hoc analysis of the same patients who were treated for a further year applying the newer CLAD classification system over an extended 7-year period of observation. ${ }^{127}$ The treated patients were less likely to develop CLAD at any stage compared with the placebo patients ( $28 \%$ vs $51 \%$, $\mathrm{p}=0.043$ ). There were also benefits in CLAD-free survival, long-term pulmonary function and functional exercise capacity, but graft loss (re-transplantation and mortality) was similar in both groups $(53 \%$ vs $40 \%, \mathrm{p}=0.27) .{ }^{127}$ In the Corris et al paper, 46 patients with BOS following lung transplantation were randomised to receive either azithromycin, $250 \mathrm{mg}$ on alternate days (23 patients) or placebo (23 patients) over a 12-week study period in double-blind fashion. The primary end point was change in $\mathrm{FEV}_{1}$ at 12 weeks. Patients who had a rapid and severe deterioration in lung function were withdrawn from the study and received open-labelled azithromycin. On an intention-totreat (ITT) analysis, there was no significant difference between treatments in the primary outcome $(0.035 \mathrm{~L}, \mathrm{p}=0.6)$. For study completers (16 azithromycin and 17 placebo), there was a significant difference of $0.278 \mathrm{~L}(\mathrm{p} \leq 0.001)$. In addition, 9/23 ITT patients in the azithromycin group had a $\geq 10 \%$ gain in $\mathrm{FEV}_{1}$ from baseline while no patient in the placebo group had $\geq 10 \%$ gain in $\mathrm{FEV}_{1}$ while on placebo $(\mathrm{p}=0.002) .{ }^{126}$ This latter observation, previously noted in other studies, ${ }^{128}$ suggests a subgroup of BOS patients who exhibit a response to azithromycin and is one of the drivers for recommending the re-classification of CLAD following lung transplantation noted above.

\section{Evidence summary}

The evidence for macrolides in BOS following lung transplantation covers both prophylactic use to prevent BOS and treatment following the occurrence of BOS. In both areas, there are single RCTs and a number of case series. The overall quality of the evidence is at best modest; however, BOS is a devastating complication of lung transplantation, so any intervention that offers the chance of prevention, reversal or stabilisation is welcome. Longterm macrolide use is a low-risk intervention. On this basis, we can make two recommendations. (Low)

\section{Recommendations}

- Low-dose, long-term azithromycin (250 mg thrice weekly) could be considered to prevent the occurrence of BOS post lung transplantation. (Conditional)

- Low-dose azithromycin $(250 \mathrm{mg}$ alternate days for a trial period of 3 months) could be considered to treat BOS occurring in lung transplant recipients. (Conditional)

\section{Macrolides in BOS following HSCT}

There are a number of case series and one RCT examining the role of macrolides (sometimes in combination with other emerging therapies) in BOS following HSCT. Khalid et al reported prospectively from Saudi Arabia on a series of eight 
HSCT patients with BOS diagnosed on the basis of lung function and high-resolution CT scanning who were treated with azithromycin for 12 weeks. All patients showed improvement in $\mathrm{FEV}_{1}$ and all but one in FVC following treatment. The changes were statistically $\left(\mathrm{FEV}_{1}, \mathrm{p}=0.0052 ; \mathrm{FVC}, \mathrm{p}=0.0067\right)$ and clinically (FEV $21.6 \%$, FVC 20.6\%) significant. ${ }^{129}$ Norman et al described a retrospective, single-centre series of eight HSCT patients in North America with newly diagnosed BOS who were treated with a combination of fluticasone (inhaled), azithromycin and montelukast (the FAM regime) in addition to a more rapid taper in systemic corticosteroids than was normal practice. The intention was to reduce systemic corticosteroid exposure in these patients. Comparison with historical controls (HSCT patients with BOS treated with much higher overall doses of systemic steroids) suggested equivalent efficacy in maintaining lung function. ${ }^{130}$ Jo et al from South Korea, in a retrospective study, described the effect of prophylactic azithromycin in reducing the incidence of BOS in a series of 100 HSCT patients compared with over 1000 similar patients who did not receive prophylactic azithromycin. The incidence of BOS in those who received azithromycin was $12 \%$ compared with $6.4 \%$ in those who did not. Subsequent multivariate analysis did not suggest that prophylactic azithromycin was associated with the development of BOS. ${ }^{131}$ In a multicentre open-labelled prospective study, Williams et al examined the effect of the FAM regimen on new-onset BOS in HSCT patients. The primary end point was treatment failure by 3 months defined as an absolute decline in $\%$ predicted $\mathrm{FEV}_{1}$ of $>$ or $=10 \%$. Thirty-six patients from 10 centres were studied. Two patients (6\%) had treatment failure compared with $40 \%$ of historical controls. ${ }^{132}$ The single-centre RCT from Lam et al in Honk Kong compared the effect of 12 weeks' treatment with daily azithromycin, $250 \mathrm{mg}$, compared with placebo in 22 HSCT patients with BOS. Primary outcomes were changes in QOL (SGRQ) and lung function $\left(\mathrm{FEV}_{1}, \mathrm{FVC}\right.$ and FEF 25-75) measured monthly and 1 month after completion of treatment. The authors reported no change in lung function or SGRQ although the QOL data were analysed in an unusual fashion. ${ }^{133}$

\section{Evidence summary}

The literature for macrolides in BOS following HSCT is limited. There is only one RCT which has significant methodological limitations. The majority of case series are small and half are retrospective. There are differing diagnostic criteria for BOS and in some studies multiple drugs including azithromycin are used. Several authors comment on the difficulty of performing studies in this patient group where the condition is a rare complication of HSCT (making it difficult to gather high patient numbers) with devastating consequences (making it difficult to justify and recruit to a placebo limb). This situation is discussed in detail in an editorial. ${ }^{134}$ There is insufficient evidence for us to make a recommendation.

\section{Macrolides for other causes of bronchiolitis obliterans}

There are no published studies looking at macrolide treatment for other causes of bronchiolitis obliterans. There is a single case report of bronchiolitis obliterans following dust exposure on $9 / 11$ with response to azithromycin ${ }^{135}$ and one recent review article suggested a possible role for macrolides in the treatment of bronchiolitis obliterans associated with rheumatoid arthritis. ${ }^{136}$

\section{SECTION 8: THE USE OF MACROLIDES FOR OTHER RESPIRATORY CONDITIONS \\ Cough}

Chronic cough, defined as a cough lasting more than 8 weeks, ${ }^{137}$ is a very common symptom, with an estimated prevalence in total of $9.6 \%$ of the population, with variations according to geographical area from as high as $12.7 \%$ in Europe to $4.4 \%$ in Asia. ${ }^{138}$ It has been reported as the the most common symptom for which patients seek medical attention. ${ }^{139}$ It occurs most commonly in women in the fifth and sixth decades of life, and has been associated with significant impacts on physical and psychological health. ${ }^{140} 141$ The most common causes of chronic cough remain cough variant asthma, postnasal drip and gastrooesophageal reflux disease, with around $40 \%$ remaining of 'unknown cause' after investigation. ${ }^{141}$

When an underlying cause of chronic cough is not identified, or when cough is resistant to prolonged treatment of the underlying cause, a number of treatments have been assessed to attempt to reduce cough frequency, reduce symptoms and improve QOL. These include randomised trials demonstrating the utility of lowdose opioids ${ }^{142}$ and gabapentin. ${ }^{143}$ Amitriptyline has been trialled (non-randomised) in cough with some success. ${ }^{144}$

Macrolides have been used in an attempt to reduce the symptoms or frequency of chronic cough. The rationale for their potential use in chronic cough includes impact on neutrophilic airways inflammation which is raised in some patients with chronic cough, ${ }^{145}$ and has been shown to be decreased with use of macrolides, ${ }^{34} 101$ and the potential anti-inflammatory effects of macrolides, independent of their antimicrobial actions. ${ }^{146}$

\section{Evidence base}

\section{Included studies}

The search strategy revealed a total of 10 papers, which included two randomised trials, three review articles, three retrospective studies on sino-bronchial syndrome in far eastern populations, one validation study of a cough tool and one animal study.

Only the two, blinded placebo controlled randomised studies (Yousaf et al 2010 and Hodgson et al 2016) (147 $148^{148}$ were included in the formulation of this guideline, with other studies rejected on the basis of being out of scope (sino-bronchial syndrome only, validation study, animal study) or irrelevant to the PICO question.

\section{Outcomes and interventions}

The outcomes assessed in the two randomised trials were varied, and heterogeneity in the outcomes (and interventions) meant that any form of meaningful data synthesis was not possible. Outcomes included number of coughs/24 hours, change in the LCQ, dose of capsaicin challenge to cause two coughs (C2) or five coughs (C5), Visual Analogue Score $100 \mathrm{~mm}$ for cough, number of colonyforming units in sputum for microbiology and cough severity.

Yousaf $e t$ al assessed the effect of erythromycin $250 \mathrm{mg}$ once daily for 12 weeks on the primary outcome (log change in 24 hours cough frequency from baseline) in 30 patients, ${ }^{147}$ while Hodgson et al assessed the effect of $250 \mathrm{mg}$ azithromycin thrice weekly for 8 weeks on LCQ score (primary) in 44 patients. ${ }^{148}$

\section{Evidence summary}

Yousaf et al demonstrated no significant difference in cough frequency between the intervention and placebo groups (mean difference in log change of cough frequency $1.1,95 \%$ CI 0.7 to $1.5, \mathrm{p}=0.59$ ), despite a significant change in the sputum neutrophil count at 12 weeks, although at other time points no difference was found. ${ }^{147}$ 
Hodgson et al demonstrated no significant difference between placebo and intervention in LCQ, nor for any secondary outcome measurements. A subgroup analysis was conducted (post hoc), identifying a subgroup of responders to azithromycin therapy (ie, those with a concomitant diagnosis of asthma, mean LCQ improvement compared with placebo 5.77 ; 95\% CI 2.75 to 8.79 ). However, given the small size of this group (18 patients in total) and post hoc nature of the subgroup, it is highly likely that this analysis suffers with bias. ${ }^{148}$

\section{Side effects and adverse events}

Side-effect profiles were published in both trials; Yousaf et al reported two patient withdrawals on erythromycin $250 \mathrm{mg}$ per day for 12 weeks, one of whom reported dizziness which resolved. ${ }^{147}$ Hodgson et al reported one withdrawal on azithromycin due to GI side effects. In the study as a whole, there were no statistically significant differences in side effects between intervention and control group (eight patients with GI side effects in the intervention group vs five in the placebo group). ${ }^{148}$

\section{Evidence statements}

Long-term macrolide antibiotics are ineffective in improving any outcomes in chronic cough, accepting that only two small RCTs have been conducted (totalling only 72 patients). These outcomes include those proven to be of importance in chronic cough such as the LCQ, number of coughs and $100 \mathrm{~mm}$ VAS for cough. (Low)

\section{Recommendations}

- Long-term macrolide antibiotics should not be used to manage patients with unexplained chronic cough. (Conditional)

\section{Research recommendation}

High-quality, adequately powered randomised trials are needed to assess the effect of macrolides on outcomes of importance in chronic cough.

\section{Organising pneumonia}

Organising pneumonia (OP) is a clinicopathological syndrome associated with characteristic patterns on lung imaging and biopsy which represents an aberrant healing response to injury within the small airways and alveolar spaces. CT scanning typically shows patchy consolidation with air bronchograms, typically in subpleural locations, which may appear migratory on serial imaging. Ground glass change, reversed halo (atoll) sign, reticulation or nodules may also be seen. Histological features of OP include fibroblasts and inflammatory cells embedded in extracellular matrix within the small airways and alveoli, often forming polypoid masses known as Masson bodies. OP can occur in a variety of conditions including infection, connective tissue disease, malignancy, following radiotherapy, drug reactions and immunodeficiency. If no cause can be found on exhaustive testing the term cryptogenic organising pneumonia (COP) (previously called bronchiolitis obliterans organising pneumonia) is used. COP is a rare disease (annual incidence $\sim 1: 100000$ ) that presents with breathlessness and cough over weeks or months, often accompanied by fever, myalgia and elevated blood inflammatory markers. Lung function tests typically show restriction and impaired gas transfer.

The prognosis of COP is good, particularly when consolidation is the primary pattern on CT. The natural history is spontaneous remission in many untreated cases. ${ }^{149}$ In non-remitting or progressive COP, oral corticosteroids have been used often in reported case series. ${ }^{150}$ There have been no placebo-controlled trials of steroid therapy, but is believed that there is an autoimmune aetiology in a proportion of cases and that steroids speed resolution. In steroid-responsive cases, relapse may occur after stopping therapy.

\section{Evidence summary}

The evidence base for macrolide therapy in OP consists of case reports and small case series totalling fewer than 50 patients. ${ }^{151-161}$ Macrolides were used either first-line in untreated patients or less commonly, as an add-on therapy with oral steroids. When used first-line, no studies described an initial observation period to identify self-resolving disease. Clinical outcome reporting is variable, but overall the published studies report clinical improvement in response to macrolide therapy. ${ }^{162}$ The risk of publication bias is high. Because the natural history of untreated COP is often self-resolution, in the absence of placebo-controlled trials, no conclusions can be made about the efficacy of macrolide therapy.

There is insufficient evidence to make a recommendation.

\section{Diffuse panbronchiolitis}

Diffuse panbronchiolitis (DPB) was defined as a specific clinicopathological entity in the 1960 s, being characterised histologically by multiple micronodular lesions consisting of chronic inflammatory cells infiltrating bronchiolar walls. ${ }^{163} 164$ It has almost exclusively been reported in East Asian countries, notably Japan, but also others such as China and South Korea. ${ }^{163}$ Relatively few cases have been diagnosed in Caucasian and other populations. ${ }^{163165166}$

DPB was one of the first respiratory conditions for which it was identified that a regular macrolide, erythromycin, at a lower dose than usually used in therapy, could be associated with notably improved outcomes for sufferers. This was discovered in the early 1980s, although there has been a paucity of published rigorous and well-designed placebo-controlled trials subsequently to test the hypothesis, with further supporting evidence largely in the form of (usually small) case series and observational studies. ${ }^{163} 167$ The largest published study was retrospective, involving nearly 500 Japanese patients with DPB. ${ }^{168}$ These patients were divided into three cohorts on the basis of likely date of diagnosis, which were then compared with respect to outcome. There was a statistically significant survival advantage $(p<0.0001)$ to being in the third cohort and thereby diagnosed between 1985 and 1990. This was ascribed as being directly related to when the beneficial role of low-dose erythromycin in DPB had first been reported. ${ }^{168}$ To provide support for this, the records of this third cohort of 87 patients were analysed in more detail-and there was a significantly higher survival rate at 5 years of those who were known to have received erythromycin compared with those who had not $(p=0.01) .{ }^{168}$ Moreover, those who had not received erythromycin had a similar 5 -year survival rate to those in the first cohort, who had been diagnosed between 1970 and 1979, and therefore before the first reports of benefit with such a strategy. ${ }^{163168}$ DPB case series studies have also shown the effect of low-dose macrolide therapy in controlling clinical symptoms, such as by reducing sputum production, and objectively improving lung function. ${ }^{167}$

The benefits of using low-dose macrolide therapy in the setting of DPB have been endorsed by clinical guidelines developed in the Far East. ${ }^{163}$ However, due to the current rarity of this condition in the UK, the GDG felt that it was not warranted for BTS to conduct a comprehensive evidence-based review and 
develop specific recommendations regarding the role of macrolides in managing DPB.

\section{SECTION 9: SAFETY ISSUES Gastrointestinal effects}

Gastrointestinal (GI) side effects of macrolides have been recognised since the introduction of erythromycin in 1952. Although initially thought to be due to alterations in gut flora, it is now clear that macrolides act through interactions with motilin receptors in the gut. ${ }^{169} 170$ In humans, macrolides potentiate gastric and small bowel motility, ${ }^{171-175}$ increase lower oesophageal sphincter pressure, ${ }^{176} 177$ and influence colonic transit and gall bladder function. ${ }^{178}$

The prokinetic effects of macrolides on the GI tract has led to their therapeutic use in conditions characterised by reduced GI motility including diabetic gastroparesis, ${ }^{179}$ anorexia nervosa, ${ }^{180}$ colonic pseudo-obstruction, postoperative ileus ${ }^{181}$ and in critical care patients. ${ }^{182}$

GI symptoms are the most commonly reported side effects of macrolides occurring in up to $70 \%$ of patients taking erythromycin, ${ }^{183}$ less commonly with clarithromycin and azithromycin. Symptoms include nausea, vomiting, abdominal pain, diarrhoea and anorexia. Commenting on 11 studies of long-term, low-dose macrolide treatment in various chronic respiratory diseases in nearly 1000 children and adults, Altenburg et $a l^{23}$ found that mild to moderate GI complaints had been reported which hardly ever caused study drug discontinuation.

Thus, although GI symptoms are common and predictable, they are rarely sufficiently troublesome to necessitate discontinuation of therapy. Dose reduction may improve tolerability.

\section{Good practice points}

$\checkmark$ Prior to initiating low-dose macrolide therapy, patients should be warned of the possibility of GI side effects.

$\checkmark$ GI side effects may be ameliorated by dose reduction although this may also reduce clinical efficacy.

$\checkmark$ Clinicians should carefully consider the risk-to-benefit balance when considering therapy for those with preexisting GI symptomatology.

\section{Cardiac effects}

Macrolide antibiotics, along with a wide range of other pharmacological agents, have the potential to interfere with conduction in cardiac tissue and hence to cause dangerous cardiac arrhythmias and sudden cardiac death. ${ }^{184}$ Macrolides can both directly prolong the QT interval ${ }^{185}$ and also inhibit the metabolism of other proarrhythmogenic drugs by acting on cytochrome P450 in the liver.

In recent years, there have been reports of an increased risk of cardiovascular death in patients treated with short, oral antibacterial courses of azithromycin ${ }^{110}$ and of no increased risk of death in a younger group of similarly treated patients. ${ }^{186}$ Discussion of these seemingly contradictory findings commented that the group of patients with increased risk exhibited a high level of baseline risk and comorbidity. ${ }^{187}$ Factors increasing the risk of malignant arrhythmias with macrolides are well recognised and include age $>80$, female gender, heart disease, use of other QT prolonging medication, reduced drug elimination, bradycardia, prolonged QT interval before therapy and genetic predisposition. ${ }^{188}$ These events are rare; 85 deaths for each 1 million courses prescribed in the increased risk group described above, ${ }^{110}$ so it is not surprising that such events do not appear in the literature of long-term, low-dose macrolide use.
A helpful review article for the American COPD Clinical Research Network describes a pragmatic approach to risk stratification when considering long-term, low-dose macrolide therapy. ${ }^{189}$ The authors combined history taking targeting evidence of heart failure, episodes of hypokalaemia, a family history of long QT syndrome, or use of other medications known to prolong the QT interval together with a pre-treatment ECG and an ECG 1 month after starting therapy. With this approach in a COPD population entering a macrolide trial, $7.1 \%$ were excluded on history and $1.6 \%$ because of a prolonged baseline QTc. A further $0.8 \%$ were withdrawn after developing QTc prolongation with treatment or on placebo. Using such a strategy, exclusions in asthma and bronchiectasis populations are likely to be fewer.

\section{Good practice points}

$\checkmark$ Prior to initiating low-dose macrolide therapy, patients should be asked if they have a history of heart disease, previous low serum potassium measurements, a slow pulse rate, a family history of sudden death or known prolonged QT interval. Patients with such a history should not receive low-dose macrolide therapy without careful consideration and counselling of the increased risk of adverse cardiac effects.

$\checkmark \quad$ Prior to initiating low-dose macrolide therapy, a drug history looking for agents that might prolong the QTc interval should be sought (see online supplementary appendices 3 and 4). Patients taking such agents should not receive lowdose macrolide therapy.

$\checkmark$ Prior to initiating low-dose macrolide therapy, an ECG should be performed to exclude a prolonged QTc interval defined as $>450 \mathrm{~ms}$ for men and $>470 \mathrm{~ms}$ for women (see methodology in online supplementary appendices 3 and 4). Patients with a prolonged QTc interval should not receive low-dose macrolides.

$\checkmark$ One month after initiating low-dose macrolide therapy, a second ECG should be performed to exclude the development of a prolonged QTc interval. Patients who develop a prolonged QTc interval on low-dose macrolides should stop the macrolide.

$\checkmark \quad$ If any new drug that could potentially prolong QTc time is started or if dose increases are made repeat ECG assessment.

\section{Ototoxicity}

Macrolide ototoxicity is well recognised usually causing a dosedependent, reversible, sensorineural hearing loss (SNHL). ${ }^{23}$ The only systematic review of this topic was published recently ${ }^{190}$ including data from three prospective studies. In these studies including 93 patients, 14 (15\%) developed SNHL confirmed by audiometry following exposure to macrolide antibiotics. All but one recovered on cessation of the drug. Earlier studies of longterm macrolide use for the treatment of mycobacterial infections have also reported reversibility of ototoxicity following either withdrawal ${ }^{191} 192$ or dose reduction ${ }^{193}$ of the macrolide. Albert reported hearing decrements in 25\% of a COPD population (vs $20 \%$ in the placebo group) with normal hearing at the outset of 1 year of low-dose azithromycin. ${ }^{93}$ Hearing subsequently improved in 34\% who discontinued treatment, but also in 32\% who continued due to protocol errors. Similar proportions of placebo-associated hearing loss also improved with or without drug withdrawal. The authors of this study felt in discussion that their criteria for defining hearing loss were too stringent and that the incidence of hearing decrements were overestimated. 
Overall, the literature suggests that for low-dose, long-term macrolide use the incidence of ototoxicity is rare and almost always reversible. It would seem sensible to assess potential recipients for hearing impairment or difficulties with balance prior to initiating therapy.

\section{Good practice point}

$\checkmark$ Prior to initiating low-dose macrolide therapy patients should be asked if they have a history of hearing or balance difficulties. Such patients should be made aware of the potential for a further, almost always reversible, deterioration in hearing or balance with macrolide therapy. Patients with pre-existing hearing or balance difficulties who wish to proceed with treatment should be asked to report any change in hearing or balance promptly.

\section{Other side effects}

A low rate (1\%-5\%) of asymptomatic elevation of serum aminotransaminase levels is known to occur with any of the four orally absorbed macrolide antibiotics. The elevation is generally mild to moderate in degree and rarely requires dose modification or discontinuation. More seriously, a cholestatic picture can occur which carries a higher risk of permanent liver damage. ${ }^{194} 195$ Monitoring liver function at the start of therapy seems a sensible precaution.

\section{Good practice points}

$\checkmark$ Prior to initiating low-dose macrolide therapy, baseline liver function tests (LFTs) should be checked.

$\checkmark \quad$ LFTs should be checked after 1 month of treatment and then every 6 months thereafter for the duration of therapy.

\section{Prescribing off-label}

It is important to be aware that none of the macrolide antibiotics have a product license in the UK for the indication of long-term, low-dose usage as immunomodulatory agents. Their prescription in this context is thus off label. Clinicians should make patients aware of this and follow GMC guidance in this area which states that "decisions should be made in collaboration with the patient by discussing the options with them and ensuring that they have sufficient information about the medicine to allow them to make an informed decision". An example of an information leaflet used to support such discussions is shown in online appendix 4.

\section{SECTION 10: ANTIMICROBIAL RESISTANCE}

Antimicrobial agents are different to other medications, as they can be associated with undesirable consequences not only in the patient receiving the treatment, but also for other individuals - both at the time and potentially at much later dates in the future. Exposure of bacteria to an antibiotic may be associated with subsequent alterations in those bacteria such that they become resistant to its effect. This means that the antibiotic loses its therapeutic value. If the antibiotic exposure, that is, with its associated selection pressure, is stopped such resistant bacteria do not necessarily revert back to being susceptible in future. The encoding mechanism of the bacterial resistance to an antibiotic's effect may be located on mobile genetic elements, such as plasmids or transposons, which will then be inherited by the future progeny of that bacterium, and may also be transferred to other strains of the same species, and indeed to other species. Such mobile genetic elements may carry the gene(s) encoding resistance to the specific antimicrobial agent, or its class, being used; and also resistance mechanisms to other, completely unrelated, antimicrobial agents/classes- that is, conferring resistance to multiple different agents. These resistant bacterial strains can then be transmitted to other individuals or establish persisting reservoirs in the environment. The resistance mechanism does not need to evolve in the pathogenic organism(s) primarily being targeted by the antimicrobial treatment. Other bacterial species, of low or no virulence, may develop such mechanisms to survive in their antibiotic-exposed milieu, such as the GI or upper respiratory tracts, but may then transmit the means to other, more virulent, species. The rise in antimicrobial resistance is of national and international concern. ${ }^{196} 197$ Not all bacteria are able to develop resistance to all antimicrobials to which they are naturally susceptible. However, this can then lead to the disappearance of some non-pathogenic commensal, 'good', bacterial species in any exposed microbiome; and their replacement by other organisms which are resistant, either intrinsically or by acquisition, which in turn may lead to subsequent infections. Such a shift in pathogens could include meticillin-resistant Staphylococcus aureus, Clostridium difficile, Gram-negative bacilli and yeasts. These ecologically adverse effects have been termed the 'collateral damage' associated with antibiotic use. ${ }^{198}$

The macrolides, such as erythromycin, clarithromycin and azithromycin, inhibit RNA-dependent protein synthesis by bacteria by binding to the $50 \mathrm{~S}$ ribosomal subunit. ${ }^{199}$ Various mechanisms by which bacteria may resist their action have been elucidated, with resistance often being cross-class. ${ }^{199}$ Many Gram-negative bacteria, such as Enterobacteriaceae ('coliforms'), Pseudomonas and Acinetobacter species, show reduced permeability of their outer cell envelope to macrolides. Azithromycin is more active in vitro against a range of Gram-negative bacteria than erythromycin. This is thought most likely due to the molecule being able to penetrate such bacterial cells more effectively. $^{200}$ Organisms, such as S. aureus and coagulasenegative staphylococci, may have plasmid-mediated macrolide resistance by increased efflux. ${ }^{201}$ The $50 \mathrm{~S}$ target site may be altered, reducing the binding affinity. ${ }^{202}$ This has been reported in a wide range of potential pathogens, including Streptococcus pneumoniae, Streptococcus pyogenes, S. aureus, Helicobacter pylori and Mycobacterium avium complex. ${ }^{199} 203$ The genetic mutation is mediated by erm genes, located either on transposons in the bacterial chromosome or on plasmids. Such resistance may also be associated with resistance to lincosamides such as clindamycin. Resistance may also be conferred by enzymatic inactivation, such as by phosphotransferases in S. aureus, Escherichia coli and Nocardia species. ${ }^{199}$

Rising bacterial resistance rates to macrolides have been reported globally. For S. pneumoniae isolates, it can vary markedly between countries, ranging from less than $10 \%$ to over $90 \%{ }^{203}$ Extensive macrolide use provides a strong selective pressure for the spread of macrolide resistance in pneumococci. $^{203} 204$ There is a notable trend for S. pneumoniae isolates, which are resistant to penicillin, also to be resistant to macrolides-in the USA, there are reports of $30 \%$ of S. pneumoniae isolates overall being erythromycin resistant, but virtually $70 \%$ of high-level penicillin-resistant isolates also exhibiting erythromycin resistance. ${ }^{199} 205$ However, it should be recognised that the increasing use of pneumococcal conjugate vaccines, which include serotypes commonly associated with macrolide resistance such as serotype 14 in the 7 -valent and also serotype 19A in the 13-valent, can then be followed by marked shifts in the prevalence of macrolide-resistant S. pneumoniae isolates. ${ }^{203} 206$ S. pyogenes isolates showing resistance to erythromycin were first reported in the UK in 1955, with resistance rates rising in the 1980s-1990s. ${ }^{199} 207$ However, reduction in resistance rates with reduced macrolide usage has also been found. For instance, 
Finland likewise experienced a rise in macrolide resistance in the 1980s, which was temporally associated with a rise in macrolide use; however, after nationwide policies were introduced to restrict their use in treating respiratory and soft-tissue infections after 1991, the resistance rate fell in clinical isolates of $S$. pyogenes from $16.5 \%$ in 1992 to $8.6 \%$ in $1996 .{ }^{208}$ Similar to S. pneumoniae, erythromycin resistance rates are much higher in $S$. aureus isolates that are meticillin resistant compared with those that are meticillin susceptible. ${ }^{199}$ The emergence of erythromycin resistance in $S$. aureus, isolated from individual patients on macrolide treatment, has been known since the discovery of the antibiotic. ${ }^{209}$

As to be expected, increased macrolide resistance has been found in studies evaluating the impact of long-term macrolide use in the setting of chronic lung diseases. ${ }^{108}{ }^{210} \mathrm{~A}$ recent metaanalysis of azithromycin use found that, while overall colonisation rates by potential pathogens, such as $S$. pneumoniae, Haemophilus influenzae and Moraxella catarrhalis, decreased, the risk of macrolide resistance, macrolide resistance among such pathogens rose 2.7 -fold in patients receiving azithromycin when compared with placebo. ${ }^{211}$ However, it should also be acknowledged that for any individual patient, the actual clinical impact of the presence of a macrolide-resistant respiratory pathogen, such as S. pneumoniae, at least in the short term, is not clear. ${ }^{212}$

Organisms for which the macrolides are a key pillar of treatment, but in which macrolide resistance can emerge, notably if any macrolide treatment is not accompanied by two or three other active agents in combination, is of particular concern in the use of long-term (ie, months to years) courses of macrolides. Such pathogens include many non-tuberculous mycobacterial (NTM) species, notably the slower growing such as M. avium complex. Such organisms may develop resistance by single point mutations, for example, in the $23 \mathrm{~S}$ rRNA gene. It is well recognised that the long-term success rate of medically treating macrolide-resistant NTM disease is very poor, and much worse than for treating a susceptible organism with a combination including an effective macrolide. ${ }^{213}$ Current NTM infection should be managed with reference to the BTS Guidelines for the management of non-tuberculous mycobacterial pulmonary disease..$^{214}$

The macrolides, notably azithromycin, also have significant applications outside the respiratory setting-including in regards to some sexually transmitted diseases (STDs), GI infections and zoonoses. ${ }^{200} 215$ One needs to be mindful that their value in such indications is affected by macrolide resistance and also by the rising bacterial resistance rates to other antibiotic classes. Circulating strains of Neisseria gonorrhoeae now show resistance to a range of antibiotic classes, to which this organism was initially susceptible. In consequence, the recent standard first-line regimen for suspected gonorrhoea has been ceftriaxone and azithromycin ${ }^{215}$; however clinical isolates of $\mathrm{N}$. gonorrhoea also resistant to both these agents have now been described. ${ }^{200} 216-218$ The latest UK guidelines, published January 2019 , now recommend therapy with ceftriaxone alone as first choice for cases where organism susceptibilities are not known, partly on the grounds of concern of rising macrolide resistance, both among $N$. gonorrhoeae but also in other organisms causing STDs. ${ }^{219}$ Azithromycin is also a recommended first-line therapy for other STDs such as chlamydia (Chlamydia trachomatis), chancroid (Haemophilus ducreyi) and donovanosis (Klebsiella granulomatis). ${ }^{200} 215$ Rising fluoroquinolone resistance rates means that macrolides are the empirical treatment of choice for diarrhoea due to Campylobacter; and, in the form of azithromycin, a first-line option for enteric fever acquired in Asia. ${ }^{200} 217$

Any indications for the macrolide class of antibiotics have been classified, in the main, in the 'Watch' group of antibiotics by WHO; along with six other classes. ${ }^{220}$ This categorisation consists of three groups: 'Access', 'Watch' and 'Restrict', the AWaRe classification. The uses of antibiotics in the Watch group should be carefully monitored to ensure they are in accordance with recommended indications. It should be recognised that the available evidence for the potential benefits and harms of longterm macrolide therapy will not capture all the associated risks of increasing antimicrobial resistance. Such studies are not designed to do so, and are too short in timescale and too restricted in patients, and organisms, being studied.

Virtually any antibiotic may be associated with diarrhoea; notably if by precipitating $C$. difficile disease. Although the macrolides appear relatively rare among antibiotic classes as being directly associated with $C$ difficile; clearly if their prior use has led to a pathogen shift, then use of an alternative antibiotic may be required which is more often associated with subsequent C. difficile disease. ${ }^{198} 221$

Antibiotics have been shown to reduce the microbial diversity and alter composition of microbiomes in murine and human studies. ${ }^{222-224}$ Such effects may be greater, and more prolonged, with macrolides than for some other antibiotics classes, such as penicillins. ${ }^{222}$ In children at least, receipt of macrolides, and the associated changes in microbial commensal flora, have been linked with other health outcomes, such as asthma and being overweight. $^{222}$ This illustrates that if there are identified benefit(s) to offering certain patients with specific chronic respiratory conditions, a macrolide as long-term supportive therapy; the known, and as yet unknown, risks of associated collateral damage should also be carefully considered, by both macrolide prescriber and macrolide consumer.

\section{Good practice points}

$\checkmark$ The risks associated with increasing antimicrobial resistance should be discussed with patients prior to starting low dose macrolide therapy. Patients should understand the risk that there may not be an effective antibiotic for them, or someone else, when needed in the future.

$\checkmark$ Prior to initiating low-dose macrolide monotherapy, patients should be asked if they have a history of previous or current NTM infection or disease. Current NTM infection should be managed with reference to BTS guidance and precludes low-dose macrolide monotherapy. Successfully treated NTM disease should not preclude low-dose macrolide monotherapy.

$\checkmark$ If there is any clinical suspicion of possible NTM disease, patients should be screened via examination of sputum samples prior to starting therapy. If positive for recognised potential pathogenic species, low-dose macrolide prophylaxis is contraindicated.

Contributors All authors drafted and approved the final version of the manuscript. DS and IDR were the lead authors with overall responsibility for the manuscript.

Funding The authors have not declared a specific grant for this research from any funding agency in the public, commercial or not-for-profit sectors.

Disclaimer Healthcare providers need to use clinical judgement, knowledge and expertise when deciding whether it is appropriate to apply recommendations for the management of patients. The recommendations cited here are a guide and may not be appropriate for use in all situations. The guidance provided does not override the responsibility of healthcare professionals to make decisions appropriate to the circumstances of each patient, in consultation with the patient and/or their guardian or carer. 
Competing interests BTS Declarations of Interest forms have been completed by all members for each year they were part of the GDG. Details of these forms can be obtained from BTS Head Office.

Patient consent for publication Not required.

Provenance and peer review Not commissioned; externally peer reviewed.

Data availability statement Supporting information is available on the BTS website (www.brit-thoracic.org.uk).

\section{ORCID iDs}

Simon Paul Hart http://orcid.org/0000-0003-4606-9559

Philip J Mitchelmore http://orcid.org/0000-0002-7850-3068

\section{REFERENCES}

1 Guyatt G, Oxman AD, Akl EA, et al. GRADE guidelines: 1. Introduction—GRADE evidence profiles and summary of findings tables. J Clin Epidemiol 2011;64:383-94.

2 T Hill A, L Sullivan A, D Chalmers J, et al. British Thoracic Society guideline for bronchiectasis in adults. Thorax 2019;74:1-69.

3 NICE. Cystic fibrosis: diagnosis and management. Contract No: NG78. NICE, 2017.

4 NICE. Asthma: diagnosis, monitoring and chronic asthma management. Contract No: NG80. NICE, 2017

5 NICE. Chronic obstructive pulmonary disease (acute exacerbation): antimicrobial prescribing. Contract No: NG114. NICE, 2018.

6 NICE. Bronchiectasis (non-cystic fibrosis), acute exacerbation: antimicrobial prescribing. NG117[Guideline]. London: NICE, 2018. https://www.nice.org.uk/ guidance/ng117

7 NICE. Chronic obstructive pulmonary disease in over 165: diagnosis and management. Contract No: NG115. London: NICE, 2018.

8 Global Initiative for Chronic Global Obstructive Lung Disease. Global strategy for the diagnosis, management and prevention of chronic obstructive lung disease, 2019 Available: www.gold.copd.org

9 Wedzicha JA, Calverley PMA, Albert RK, et al. Prevention of COPD exacerbations: a European Respiratory Society/American Thoracic Society guideline. Eur Respir J 2017:50:1602265.

10 Herath SC, Normansell R, Maisey S, et al. Prophylactic antibiotic therapy for chronic obstructive pulmonary disease (COPD). Cochrane Database Syst Rev 2018;10:CD009764.

11 Hnin K, Nguyen C, Carson-Chahhoud KV, et al. Prolonged antibiotics for noncystic fibrosis bronchiectasis in children and adults. Cochrane Database Syst Rev 2015;209.

12 Normansell R, Sayer B, Waterson S, et al. Antibiotics for exacerbations of asthma. Cochrane Database Syst Rev 2018;6:CD002741.

13 Kelly C, Chalmers JD, Crossingham I, et al. Macrolide antibiotics for bronchiectasis. Cochrane Database Syst Rev 2018;3:CD012406.

14 Kew KM, Undela K, Kotortsi I, et al. Macrolides for chronic asthma. Cochrane Database Syst Rev 2015:CD002997.

15 Global Initiative for Asthma. GINA report 2018, global strategy for asthma management and prevention, 2018. Available: https://ginasthma.org/wp-content/ uploads/2018/04/wms-GINA-2018-report-V1.3-002.pdf [Accessed 20 May 2019].

16 Hansen MP, Scott AM, McCullough A, et al. Adverse events in people taking macrolide antibiotics versus placebo for any indication. Cochrane Database Syst Rev 2019:1:CD011825.

17 Itkin IH, Menzel ML. The use of macrolide antibiotic substances in the treatment of asthma. J Allergy 1970;45:146-62.

18 Zeiger RS, Schatz M, Sperling W, et al. Efficacy of troleandomycin in outpatients with severe, corticosteroid-dependent asthma. J Allergy Clin Immunol 1980;66:438-46.

19 Homma H, Yamanaka A, Tanimoto S, et al. Diffuse panbronchiolitis. A disease of the transitional zone of the lung. Chest 1983;83:63-9.

20 Kudoh S, Uetake T, Hagiwara K, et al. [Clinical effects of low-dose long-term erythromycin chemotherapy on diffuse panbronchiolitis]. Nihon Kyobu Shikkan Gakkai Zasshi 1987;25:632-42.

21 Koyama H, Geddes DM. Erythromycin and diffuse panbronchiolitis. Thorax 1997:52:915-8.

22 Kanoh S, Rubin BK. Mechanisms of action and clinical application of macrolides as immunomodulatory medications. Clin Microbiol Rev 2010;23:590-615.

23 Altenburg J, de Graaff CS, van der Werf TS, et al. Immunomodulatory effects of macrolide antibiotics—part 2: advantages and disadvantages of long-term, lowdose macrolide therapy. Respiration 2011;81:75-87.

24 Serisier DJ. Risks of population antimicrobial resistance associated with chronic macrolide use for inflammatory airway diseases. Lancet Respir Med 2013;1:262-74.

25 BTS/SIGN. British quideline on the management of asthma. BTS/SIGN, 2016.

26 Black PN, Scicchitano R, Jenkins CR, et al. Serological evidence of infection with Chlamydia pneumoniae is related to the severity of asthma. Eur Respir J 2000;15:254-9.

27 Gotfried MH, Jung R, Messick CR, et al. Effects of six-week clarithromycin therapy in corticosteroid-dependent asthma: a randomized, double-blind, placebo-controlled pilot study. Curr Ther Res Clin Exp 2004;65:1-12.
28 Juniper EF, Guyatt GH, Epstein RS, et al. Evaluation of impairment of health related quality of life in asthma: development of a questionnaire for use in clinical trials. Thorax 1992;47:76-83.

29 Black PN, Blasi F, Jenkins CR, et al. Trial of roxithromycin in subjects with asthma and serological evidence of infection with Chlamydia pneumoniae. Am J Respir Crit Care Med 2001:164:536-41.

30 Brusselle GG, Vanderstichele C, Jordens P, et al. Azithromycin for prevention of exacerbations in severe asthma (AZISAST): a multicentre randomised double-blind placebo-controlled trial. Thorax 2013;68:322-9.

31 Cameron EJ, Chaudhuri R, Mair F, et al. Randomised controlled trial of azithromycin in smokers with asthma. Eur Respir I 2013:42:1412-5.

32 Hahn DL, Grasmick M, Hetzel S, et al. Azithromycin for bronchial asthma in adults: an effectiveness trial. J Am Board Fam Med 2012:25:442-59.

33 Hahn DL, Plane MB, Mahdi OS, et al. Secondary outcomes of a pilot randomized trial of azithromycin treatment for asthma. PLoS Clin Trials 2006;1:e11.

34 Simpson JL, Powell H, Boyle MJ, et al. Clarithromycin targets neutrophilic airway inflammation in refractory asthma. Am J Respir Crit Care Med 2008:177:148-55.

35 Sutherland ER, King TS, Icitovic N, et al. A trial of clarithromycin for the treatment of suboptimally controlled asthma. J Allergy Clin Immunol 2010:126:747-53.

36 Tong X, Guo T, Liu S, et al. Macrolide antibiotics for treatment of asthma in adults: a meta-analysis of 18 randomized controlled clinical studies. Pulm Pharmacol Ther 2015:31:99-108

37 Amayasu H, Yoshida S, Ebana S, et al. Clarithromycin suppresses bronchial hyperresponsiveness associated with eosinophilic inflammation in patients with asthma. Ann Allergy Asthma Immunol 2000;84:594-8.

38 Coeman M, van Durme Y, Bauters F, et al. Neomacrolides in the treatment of patients with severe asthma and/or bronchiectasis: a retrospective observational study. Ther Adv Respir Dis 2011:5:377-86.

39 Evans DJ, Cullinan P, Geddes DM, et al. Troleandomycin as an oral corticosteroid steroid sparing agent in stable asthma. Cochrane Database Syst Rev 2001:CD002987.

40 Gibson PG, Yang IA, Upham JW, et al. Effect of azithromycin on asthma exacerbations and quality of life in adults with persistent uncontrolled asthma (AMAZES): a randomised, double-blind, placebo-controlled trial. Lancet 2017:390:659-68.

41 Reiter J, Demirel N, Mendy A, et al. Macrolides for the long-term management of asthma — a meta-analysis of randomized clinical trials. Allergy 2013;68:1040-9.

42 Juniper EF, O'Byrne PM, Guyatt GH, et al. Development and validation of a questionnaire to measure asthma control. Eur Respir J 1999:14:902-7.

43 Nelson HS, Hamilos DL, Corsello PR, et al. A double-blind study of troleandomycin and methylprednisolone in asthmatic subjects who require daily corticosteroids. Am Rev Respir Dis 1993;147:398-404.

44 Kamada AK, Hill MR, IKlé DN, et al. Efficacy and safety of low-dose troleandomycin therapy in children with severe, steroid-requiring asthma. J Allergy Clin Immunol 1993;91:873-82.

45 Ball BD, Hill MR, Brenner M, et al. Effect of low-dose troleandomycin on glucocorticoid pharmacokinetics and airway hyperresponsiveness in severely asthmatic children. Ann Allergy 1990:65:37-45.

46 Shoji T, Yoshida S, Sakamoto H, et al. Anti-inflammatory effect of roxithromycin in patients with aspirin-intolerant asthma. Clin Exp Allergy 1999:29:950-6.

47 Wang T, Pei FY, Song XP, et al. Clinical impacts of low-dose azithromycin on lung function and fraction of exhaled nitric oxide; concentration in bronchial asthma. Med Innovation China 2014;11:122-4

48 Kostadima E, Tsiodras S, Alexopoulos El, et al. Clarithromycin reduces the severity of bronchial hyperresponsiveness in patients with asthma. Eur Respir J 2004;23:714-7.

49 Lopes Dos Santos Santiago G, Brusselle G, Dauwe K, et al. Influence of chronic azithromycin treatment on the composition of the oropharyngeal microbial community in patients with severe asthma. BMC Microbiol 2017:17:109.

50 Anwar GA, McDonnell MJ, Worthy SA, et al. Phenotyping adults with non-cystic fibrosis bronchiectasis: a prospective observational cohort study. Respir Med 2013:107:1001-7.

51 Lonni S, Chalmers JD, Goeminne PC, et al. Etiology of non-cystic fibrosis bronchiectasis in adults and its correlation to disease severity. Ann Am Thorac Soc 2015;12:1764-70.

52 Pasteur MC, Helliwell SM, Houghton SJ, et al. An investigation into causative factors in patients with bronchiectasis. Am J Respir Crit Care Med 2000;162:1277-84.

53 Quint JK, Millett ERC, Joshi M, et al. Changes in the incidence, prevalence and mortality of bronchiectasis in the UK from 2004 to 2013: a population-based cohort study. Eur Respir J 2016:47:186-93.

54 Shoemark A, Ozerovitch L, Wilson R. Aetiology in adult patients with bronchiectasis. Respir Med 2007:101:1163-70.

55 Goeminne PC, Scheers H, Decraene A, et al. Risk factors for morbidity and death in non-cystic fibrosis bronchiectasis: a retrospective cross-sectional analysis of CT diagnosed bronchiectatic patients. Respir Res 2012;13:21.

56 Polverino E, Goeminne PC, MCDonnell MJ, et al. European Respiratory Society guidelines for the management of adult bronchiectasis. Eur Respir 」 2017;50:1700629 
57 Fouka E, Lamprianidou E, Arvanitidis K, et al. Low-dose clarithromycin therapy modulates Th17 response in non-cystic fibrosis bronchiectasis patients. Lung 2014;192:849-55.

58 Liu J, Zhong X, He Z, et al. Effect of low-dose, long-term roxithromycin on airway inflammation and remodeling of stable noncystic fibrosis bronchiectasis. Mediators Inflamm 2014;2014:1-14.

59 Burr LD, Rogers GB, Chen AC-H, et al. Macrolide treatment inhibits Pseudomonas aeruginosa quorum sensing in non-cystic fibrosis bronchiectasis. An analysis from the bronchiectasis and low-dose erythromycin study trial. Ann Am Thorac Soc 2016;13:1697-703.

60 Davies G, Wilson R. Prophylactic antibiotic treatment of bronchiectasis with azithromycin. Thorax 2004;59:540-1.

61 Wong C, Jayaram L, Karalus N, et al. Azithromycin for prevention of exacerbations in non-cystic fibrosis bronchiectasis (EMBRACE): a randomised, double-blind, placebocontrolled trial. Lancet 2012;380:660-7.

62 Altenburg J, de Graaff CS, Stienstra Y, et al. Effect of azithromycin maintenance treatment on infectious exacerbations among patients with non-cystic fibrosis bronchiectasis: the BAT randomized controlled trial. JAMA 2013;309:1251-9.

63 Serisier DJ, Martin ML, McGuckin MA, et al. Effect of long-term, low-dose erythromycin on pulmonary exacerbations among patients with non-cystic fibrosis bronchiectasis: the BLESS randomized controlled trial. JAMA 2013;309:1260-7.

64 Hill AT, Welham S, Reid K, et al. British Thoracic Society national bronchiectasis audit 2010 and 2011. Thorax 2012;67:928-30.

65 Anwar GA, Bourke SC, Afolabi G, et al. Effects of long-term low-dose azithromycin in patients with non-CF bronchiectasis. Respir Med 2008;102:1494-6.

66 Diego AD, Milara J, Martinez-Moragón E, et al. Effects of long-term azithromycin therapy on airway oxidative stress markers in non-cystic fibrosis bronchiectasis. Respirology 2013;18:1056-62.

67 Cymbala AA, Edmonds LC, Bauer MA, et al. The disease-modifying effects of twice-weekly oral azithromycin in patients with bronchiectasis. Treat Respir Med 2005;4:117-22.

68 Tsang KW, Ho PI, Chan KN, et al. A pilot study of low-dose erythromycin in bronchiectasis. Eur Respir J 1999;13:361-4.

69 Fan L-C, Lu H-W, Wei P, et al. Effects of long-term use of macrolides in patients with non-cystic fibrosis bronchiectasis: a meta-analysis of randomized controlled trials. BMC Infect Dis 2015;15:160.

70 Wu Q, Shen W, Cheng H, et al. Long-term macrolides for non-cystic fibrosis bronchiectasis: a systematic review and meta-analysis. Respirology 2014;19:321-9.

71 Zhuo G-Y, He Q, Xiang-Lian L, et al. Prolonged treatment with macrolides in adult patients with non-cystic fibrosis bronchiectasis: meta-analysis of randomized controlled trials. Pulm Pharmacol Ther 2014;29:80-8. [Erratum appears in Pulm Pharmacol Ther. 2014 Oct;29(1):90; PMID: 24752188].

72 Chalmers JD, Boersma W, Lonergan M, et al. Long-term macrolide antibiotics for the treatment of bronchiectasis in adults: an individual participant data meta-analysis. Lancet Respir Med 2019;7:845-54.

73 Hill AT, Haworth CS, Aliberti S, et al. Pulmonary exacerbation in adults with bronchiectasis: a consensus definition for clinical research. Eur Respir $J$ 2017:49:1700051.

74 Chalmers JD, Goeminne P, Aliberti S, et al. The bronchiectasis severity index. An international derivation and validation study. Am J Respir Crit Care Med 2014;189:576-85.

75 Aliberti S, Masefield S, Polverino E, et al. Research priorities in bronchiectasis: a consensus statement from the EMBARC clinical research collaboration. Eur Respir J 2016;48:632-47.

76 Rodriguez-Roisin R. Toward a consensus definition for COPD exacerbations. Chest 2000;117:398S-401.

77 Suissa S, Dell'Aniello S, Ernst P. Long-term natural history of chronic obstructive pulmonary disease: severe exacerbations and mortality. Thorax 2012;67:957-63.

78 Celli BR, MacNee W, ATS/ERS Task Force. Standards for the diagnosis and treatment of patients with COPD: a summary of the ATS/ERS position paper. Eur Respir J 2004;23:932-46.

79 Di Stefano A, Caramori G, Gnemmi I, et al. T helper type 17-related cytokine expression is increased in the bronchial mucosa of stable chronic obstructive pulmonary disease patients. Clin Exp Immunol 2009;157:316-24.

80 Sethi S, Murphy TF. Infection in the pathogenesis and course of chronic obstructive pulmonary disease. N Engl J Med 2008;359:2355-65.

81 Blasi F, Mantero M, Aliberti S. Antibiotics as immunomodulant agents in COPD. Curr Opin Pharmacol 2012;12:293-9.

82 Wedzicha JA, Miravitlles M, Hurst JR, et al. Management of COPD exacerbations: a European Respiratory Society/American Thoracic Society guideline. Eur Respir J 2017;49:1600791.

83 Uzun S, Djamin RS, Kluytmans JAJW, et al. Azithromycin maintenance treatment in patients with frequent exacerbations of chronic obstructive pulmonary disease (COLUMBUS): a randomised, double-blind, placebo-controlled trial. Lancet Respir Med 2014:2:361-8.

84 Simpson JL, Powell H, Baines KJ, et al. The effect of azithromycin in adults with stable neutrophilic COPD: a double blind randomised, placebo controlled trial. PLOS One 2014;9:e105609.
85 Sethi S, Jones PW, Theron MS, et al. Pulsed moxifloxacin for the prevention of exacerbations of chronic obstructive pulmonary disease: a randomized controlled trial. Respir Res 2010;11:10.

86 Mygind LH, Pedersen C, Vestbo J, et al. A randomized, placebo-controlled 3 years study of prophylactic azithromycin in 575 patients with chronic obstructive pulmonary disease (COPD) [Abstract]. European Respiratory Society Annual Congress; September 18-22, Barcelona, Spain, 2010:5580.

87 Shafuddin E, Mills GD, Holmes MD, et al. A double-blind, randomised, placebocontrolled study of roxithromycin and doxycycline combination, roxithromycin alone, or matching placebo for 12 weeks in adults with frequent exacerbations of chronic obstructive pulmonary disease. J Negat Results Biomed 2015;14:15.

88 Pomares X, Montón C, Espasa M, et al. Long-term azithromycin therapy in patients with severe COPD and repeated exacerbations. Int I Chron Obstruct Pulmon Dis 2011;6:449-56.

89 Yamaya M, Azuma A, Takizawa $\mathrm{H}$, et al. Macrolide effects on the prevention of COPD exacerbations. Eur Respir J 2012;40:485-94.

90 Singh D, Agusti A, Anzueto A, et al. Global strategy for the diagnosis, management, and prevention of chronic obstructive lung disease: the GOLD science committee report 2019. Eur Respir J 2019:53:1900164.

91 Weatherall M, Marsh S, Shirtcliffe P, et al. Quality of life measured by the St George's respiratory questionnaire and spirometry. Eur Respir J 2009;33:1025-30.

92 Jones PW, Quirk FH, Baveystock CM. The St George's respiratory questionnaire. Respir Med 1991;85 Suppl B:25-31. discussion 3-7.

93 Albert RK, Connett J, Bailey WC, et al. Azithromycin for prevention of exacerbations of COPD. N Engl J Med 2011;365:689-98.

94 Berkhof FF, Hertog NE, Uil SM, et al. Azithromycin and cough-specific health status in patients with chronic obstructive pulmonary disease and chronic cough: a randomised controlled trial. Respir Res 2013:14:125.

95 Jones PW. St. George's respiratory questionnaire: MCID. COPD 2005;2:75-9.

96 Banerjee D, Khair OA, Honeybourne D. The effect of oral clarithromycin on health status and sputum bacteriology in stable COPD. Respir Med 2005;99:208-15.

97 He Z-Y, Ou L-M, Zhang J-Q, et al. Effect of 6 months of erythromycin treatment on inflammatory cells in induced sputum and exacerbations in chronic obstructive pulmonary disease. Respiration 2010;80:445-52.

98 Blasi F, Bonardi D, Aliberti S, et al. Long-term azithromycin use in patients with chronic obstructive pulmonary disease and tracheostomy. Pulm Pharmacol Ther 2010;23:200-7.

99 Roversi S, Corbetta L, Clini E. GOLD 2017 recommendations for COPD patients: toward a more personalized approach. COPD Research and Practice 2017;3:5.

100 Ni W, Shao X, Cai X, et al. Prophylactic use of macrolide antibiotics for the prevention of chronic obstructive pulmonary disease exacerbation: a meta-analysis. PLoS One 2015;10:e0121257.

101 Seemungal TAR, Wilkinson TMA, Hurst JR, et al. Long-term erythromycin therapy is associated with decreased chronic obstructive pulmonary disease exacerbations. Am J Respir Crit Care Med 2008;178:1139-47.

102 Suzuki T, Yanai M, Yamaya M, et al. Erythromycin and common cold in COPD. Chest 2001;120:730-3.

103 Han MK, Tayob N, Murray S, et al. Predictors of chronic obstructive pulmonary disease exacerbation reduction in response to daily azithromycin therapy. Am J Respir Crit Care Med 2014;189:1503-8.

104 Fletcher C, Peto R. The natural history of chronic airflow obstruction. Br Med J 1977:1:1645-8.

105 Vestbo J, Edwards LD, Scanlon PD, et al. Changes in forced expiratory volume in 1 second over time in COPD. N Engl J Med 2011;365:1184-92.

106 Casanova C, de Torres JP, Aguirre-Jaíme A, et al. The progression of chronic obstructive pulmonary disease is heterogeneous: the experience of the BODE cohort. Am J Respir Crit Care Med 2011;184:1015-21.

107 European Medicines Agency. Guideline on clinical investigation of medicinal products in the treatment of chronic obstructive pulmonary disease, 2012. Available: http://www.ema.europa.eu/docs/en_GB/document_library/Scientific_guideline/ 2012/08/WC500130880.pdf

108 Taylor SP, Sellers E, Taylor BT. Azithromycin for the prevention of COPD exacerbations: the good, bad, and ugly. Am J Med 2015;128:1362.e1-6.

109 Jain R, Danziger LH. The macrolide antibiotics: a pharmacokinetic and pharmacodynamic overview. Curr Pharm Des 2004;10:3045-53.

110 Ray WA, Murray KT, Hall K, et al. Azithromycin and the risk of cardiovascular death. N Engl J Med 2012;366:1881-90.

111 Lu ZK, Yuan J, Li M, et al. Cardiac risks associated with antibiotics: azithromycin and levofloxacin. Expert Opin Drug Saf 2015;14:295-303.

112 Almalki ZS, Guo JJ. Cardiovascular events and safety outcomes associated with azithromycin therapy: a meta-analysis of randomized controlled trials. Am Health Drug Benefits 2014;7:318-28.

113 Blasi F, Damato S, Cosentini R, et al. Chlamydia pneumoniae and chronic bronchitis: association with severity and bacterial clearance following treatment. Thorax 2002:57:672-6

114 Berenson CS, Garlipp MA, Grove LJ, et al. Impaired phagocytosis of nontypeable Haemophilus influenzae by human alveolar macrophages in chronic obstructive pulmonary disease. J Infect Dis 2006;194:1375-84. 
115 Look DC, Chin CL, Manzel LJ, et al. Modulation of airway inflammation by Haemophilus influenzae isolates associated with chronic obstructive pulmonary disease exacerbation. Proc Am Thorac Soc 2006;3:482-3.

116 Sethi S, Wrona C, Grant BJB, et al. Strain-specific immune response to Haemophilus influenzae in chronic obstructive pulmonary disease. Am J Respir Crit Care Med 2004;169:448-53.

117 Wu L, Skinner SJ, Lambie N, et al. Immunohistochemical staining for Chlamydia pneumoniae is increased in lung tissue from subjects with chronic obstructive pulmonary disease. Am J Respir Crit Care Med 2000;162:1148-51.

118 Brill SE, Law M, El-Emir E, et al. Effects of different antibiotic classes on airway bacteria in stable COPD using culture and molecular techniques: a randomised controlled trial. Thorax 2015;70:930-8.

119 Seemungal TAR, Hurst JR, Wedzicha JA. Exacerbation rate, health status and mortality in COPD - a review of potential interventions. Int J Chron Obstruct Pulmon Dis 2009;4:203-23.

120 Schmidt SAJ, Johansen MB, Olsen M, et al. The impact of exacerbation frequency on mortality following acute exacerbations of COPD: a registry-based cohort study. BM Open 2014;4:e006720.

121 Barker AF, Bergeron A, Rom WN, et al. Obliterative bronchiolitis. N Engl J Med 2014;370:1820-8

122 Cooper JD, Billingham M, Egan T, et al. A working formulation for the standardization of nomenclature and for clinical staging of chronic dysfunction in lung allografts. International Society for Heart and Lung Transplantation. J Heart Lung Transplant 1993;12:713-6.

123 Estenne M, Maurer JR, Boehler A, et al. Bronchiolitis obliterans syndrome 2001: an update of the diagnostic criteria. J Heart Lung Transplant 2002;21:297-310.

124 Meyer KC, Raghu G, Verleden GM, et al. An international ISHLT/ATS/ERS clinical practice guideline: diagnosis and management of bronchiolitis obliterans syndrome. Eur Respir J 2014;44:1479-503.

125 Vos R, Vanaudenaerde BM, Verleden SE, et al. A randomised controlled trial of azithromycin to prevent chronic rejection after lung transplantation. Eur Respir J 2011;37:164-72

126 Corris PA, Ryan VA, Small T, et al. A randomised controlled trial of azithromycin therapy in bronchiolitis obliterans syndrome (BOS) post lung transplantation. Thorax 2015:70:442-50.

127 Ruttens D, Verleden SE, Vandermeulen E, et al. Prophylactic azithromycin therapy after lung transplantation: post hoc analysis of a randomized controlled trial. Am J Transplant 2016;16:254-61.

128 Vanaudenaerde BM, Meyts I, Vos R, et al. A dichotomy in bronchiolitis obliterans syndrome after lung transplantation revealed by azithromycin therapy. Eur Respir J 2008;32:832-42.

129 Khalid M, Al Saghir A, Saleemi S, et al. Azithromycin in bronchiolitis obliterans complicating bone marrow transplantation: a preliminary study. Eur Respir J 2005;25:490-3.

130 Norman BC, Jacobsohn DA, Williams KM, et al. Fluticasone, azithromycin and montelukast therapy in reducing corticosteroid exposure in bronchiolitis obliterans syndrome after allogeneic hematopoietic SCT: a case series of eight patients. Bone Marrow Transplant 2011;46:1369-73.

131 Jo K-W, Yoon S, Song JW, et al. The efficacy of prophylactic azithromycin on bronchiolitis obliterans syndrome after hematopoietic stem cell transplantation. Int Hematol 2015;102:357-63.

132 Williams KM, Cheng G-S, Pusic I, et al. Fluticasone, azithromycin, and montelukast treatment for new-onset bronchiolitis obliterans syndrome after hematopoietic cell transplantation. Biol Blood Marrow Transplant 2016;22:710-6.

133 Lam DCL, Lam B, Wong MKY, et al. Effects of azithromycin in bronchiolitis obliterans syndrome after hematopoietic SCT—a randomized double-blinded placebocontrolled study. Bone Marrow Transplant 2011;46:1551-6.

134 Wolff $D$, Hildebrandt GC. Bronchiolitis obliterans: pleading for a pragmatic approach Biol Blood Marrow Transplant 2016;22:591-3.

135 Mann JM, Sha KK, Kline G, et al. World Trade Center dyspnea: bronchiolitis obliterans with functional improvement: a case report. Am J Ind Med 2005;48:225-9.

136 Shaw M, Collins BF, Ho LA, et al. Rheumatoid arthritis-associated lung disease. Eur Respir Rev 2015;24:1-16.

137 Ford AC, Forman D, Moayyedi P, et al. Cough in the community: a cross sectional survey and the relationship to gastrointestinal symptoms. Thorax 2006:61:975-9.

138 Song W-J, Chang Y-S, Faruqi S, et al. Defining chronic cough: a systematic review of the epidemiological literature. Allergy Asthma Immunol Res 2016:8:146-55.

139 Schappert SM, Burt CW. Ambulatory care visits to physician offices, hospital outpatient departments, and emergency departments: United States, 2001-02. Vital Health Stat 13 2006:1-66.

140 French CL, Irwin RS, Curley FJ, et al. Impact of chronic cough on quality of life. Arch Intern Med 1998;158:1657-61.

141 Smith JA, Woodcock A, Cough C. Chronic cough. N Engl J Med 2016;375:1544-51.

142 Morice AH, Menon MS, Mulrennan SA, et al. Opiate therapy in chronic cough. Am J Respir Crit Care Med 2007;175:312-5.

143 Ryan NM, Birring SS, Gibson PG. Gabapentin for refractory chronic cough: a randomised, double-blind, placebo-controlled trial. Lancet 2012;380:1583-9.
144 Jeyakumar A, Brickman TM, Haben M. Effectiveness of amitriptyline versus cough suppressants in the treatment of chronic cough resulting from postviral vagal neuropathy. Laryngoscope 2006;116:2108-12.

145 Yousaf N, Matos S, Birring SS, et al. Factors affecting cough frequency in a mixed population. Thorax 2009;64(Suppl IV):A151.

146 Schultz MJ. Macrolide activities beyond their antimicrobial effects: macrolides in diffuse panbronchiolitis and cystic fibrosis. J Antimicrob Chemother 2004;54:21-8.

147 Yousaf N, Monteiro W, Parker D, et al. Long-term low-dose erythromycin in patients with unexplained chronic cough: a double-blind placebo controlled trial. Thorax 2010;65:1107-10.

148 Hodgson D, Anderson J, Reynolds C, et al. The effects of azithromycin in treatmentresistant cough: a randomized, double-blind, placebo-controlled trial. Chest 2016;149:1052-60.

149 Bradley B, Branley HM, Egan JJ, et al. Interstitial lung disease guideline: the British Thoracic Society in collaboration with the Thoracic Society of Australia and New Zealand and the Irish Thoracic Society. Thorax 2008;63(Suppl 5):v1-58.

150 Cottin V, Cordier J-F. Cryptogenic organizing pneumonia. Semin Respir Crit Care Med 2012;33:462-75

151 Chang WJ, Lee EJ, Lee SY, et al. Successful salvage treatment of steroid-refractory bronchiolar COP with low-dose macrolides. Pathol Int 2012;62:144-8.

152 Ding Q-L, Lv D, Wang B-J, et al. Macrolide therapy in cryptogenic organizing pneumonia: a case report and literature review. Exp Ther Med 2015;9:829-34.

153 Hotta M. Neutrophil chemotactic activity in cryptogenic organizing pneumonia and the response to erythromycin. Kurume Med J 1996:43:207-17.

154 Ichikawa Y, Ninomiya H, Katsuki M, et al. Low-dose/long-term erythromycin for treatment of bronchiolitis obliterans organizing pneumonia (BOOP). Kurume Med $J$ 1993;40:65-7.

155 Kastelik JA, Greenstone M, McGivern DV, et al. Cryptogenic organising pneumonia. Eur Respir J 2006;28:1291.

$156 \mathrm{Oh} \mathrm{JH}, \mathrm{Oh} \mathrm{DJ}$, Koo S-M, et al. Different responses to clarithromycin in patients with cryptogenic organizing pneumonia. Tuberc Respir Dis 2015;78:401-7.

157 Pathak V, Kuhn JM, Durham C, et al. Macrolide use leads to clinical and radiological improvement in patients with cryptogenic organizing pneumonia. Ann Am Thorac Soc 2014;11:87-91.

158 Radzikowska E, Roży A, Jagus P, et al. Clarithromycin decreases IL-6 concentration in serum and BAL fluid in patients with cryptogenic organizing pneumonia. Adv Clin Exp Med 2016:25:871-8.

159 Radzikowska E, Wiatr E, Gawryluk D, et al. [Organizing pneumonia-clarithromycin treatment]. Pneumonol Alergol Pol 2008;76:334-9.

160 Stover DE, Mangino D. Macrolides: a treatment alternative for bronchiolitis obliterans organizing pneumonia? Chest 2005;128:3611-7.

161 Vaz AP, Morais A, Melo N, et al. [Azithromycin as an adjuvant therapy in cryptogenic organizing pneumonia]. Rev Port Pneumol 2011;17:186-9.

162 D'Elia T. The effectiveness of low-dose azithromycin in relapsing cryptogenic organizing pneumonia: a case report and a review of the literature. Clin Pulm Med 2016:23:179-83.

163 Azuma A, Kudoh S. Diffuse panbronchiolitis in East Asia. Respirology 2006:11:249-61.

164 Yamanaka A, Saiki S, Tamura S, et al. [Problems in chronic obstructive bronchial diseases, with special reference to diffuse panbronchiolitis]. Naika 1969;23:442-51.

165 Poletti V, Patelli M, Poletti G, et al. Diffuse panbronchiolitis observed in an Italian male. Sarcoidosis 1992:9:67-9.

166 Fitzgerald JE, King TE, Lynch DA, et al. Diffuse panbronchiolitis in the United States. Am J Respir Crit Care Med 1996:154:497-503.

167 Lin X, Lu J, Yang M, et al. Macrolides for diffuse panbronchiolitis. Cochrane Database Syst Rev 2015;1:CD007716.

168 Kudoh S, Azuma A, Yamamoto M, et al. Improvement of survival in patients with diffuse panbronchiolitis treated with low-dose erythromycin. Am J Respir Crit Care Med 1998;157:1829-32

169 Kondo Y, Torii K, Itoh Z, et al. Erythromycin and its derivatives with motilin-like biological activities inhibit the specific binding of 125I-motilin to duodenal muscle. Biochem Biophys Res Commun 1988:150:877-82.

170 Broad J, Sanger GJ. The antibiotic azithromycin is a motilin receptor agonist in human stomach: comparison with erythromycin. Br J Pharmacol 2013;168:1859-67.

171 Tomomasa T, Kuroume T, Arai H, et al. Erythromycin induces migrating motor complex in human gastrointestinal tract. Dig Dis Sci 1986;31:157-61.

172 Bortolotti M, Brunelli F, Sarti P, et al. Effects of oral clarithromycin and amoxycillin on interdigestive gastrointestinal motility of patients with functional dyspepsia and Helicobacter pylori gastritis. Aliment Pharmacol Ther 1998:12:1021-5.

173 Sifrim D, Matsuo H, Janssens J, et al. Comparison of the effects of midecamycin acetate and azithromycin on gastrointestinal motility in man. Drugs Exp Clin Res 1994:20:121-6.

174 Moshiree B, McDonald R, Hou W, et al. Comparison of the effect of azithromycin versus erythromycin on antroduodenal pressure profiles of patients with chronic functional gastrointestinal pain and gastroparesis. Dig Dis Sci 2010;55:675-83

175 Chini P, Toskes PP, Waseem S, et al. Effect of azithromycin on small bowel motility in patients with gastrointestinal dysmotility. Scand J Gastroentero/ 2012;47:422-7. 
176 Tzovaras $G$, Xynos E, Chrysos $E$, et al. The effect of intravenous erythromycin on esophageal motility in healthy subjects. Am J Surg 1996;171:316-9.

177 Pennathur A, Tran A, Cioppi M, et al. Erythromycin strengthens the defective lower esophageal sphincter in patients with gastroesophageal reflux disease. Am I Surg 1994;167:169-73. discussion 72-3.

178 Catnach SM, Fairclough PD. Erythromycin and the gut. Gut 1992;33:397-401.

179 Janssens J, Peeters TL, Vantrappen G, et al. Improvement of gastric emptying in diabetic gastroparesis by erythromycin. Preliminary studies. N Eng/ J Med 1990;322:1028-31

180 Stacher G, Peeters TL, Bergmann H, et al. Erythromycin effects on gastric emptying, antral motility and plasma motilin and pancreatic polypeptide concentrations in anorexia nervosa. Gut 1993;34:166-72.

181 Longo WE, Vernava AM. Prokinetic agents for lower gastrointestinal motility disorders. Dis Colon Rectum 1993;36:696-708.

182 Röhm KD, Boldt J, Piper SN. Motility disorders in the ICU: recent therapeutic options and clinical practice. Curr Opin Clin Nutr Metab Care 2009;12:161-7.

183 Carter BL, Woodhead JC, Cole KJ, et al. Gastrointestinal side effects with erythromycin preparations. Drug Intell Clin Pharm 1987;21:734-8.

184 van Noord C, Eijgelsheim M, Stricker BHC. Drug- and non-drug-associated QT interval prolongation. Br J Clin Pharmacol 2010;70:16-23.

185 Volberg WA, Koci BJ, Su W, et al. Blockade of human cardiac potassium channel human ether-a-go-go-related gene (hERG) by macrolide antibiotics. J Pharmacol Exp Ther 2002;302:320-7.

186 Svanström H, Pasternak B, Hviid A. Use of azithromycin and death from cardiovascular causes. N Engl J Med 2013;368:1704-12

187 Mosholder AD, Mathew J, Alexander JJ, et al. Cardiovascular risks with azithromycin and other antibacterial drugs. N Engl J Med 2013;368:1665-8.

188 Shaffer D, Singer S, Korvick J, et al. Concomitant risk factors in reports of torsades de pointes associated with macrolide use: review of the United States Food and Drug Administration adverse event reporting system. Clin Infect Dis 2002;35:197-200.

189 Albert RK, Schuller JL, COPD Clinical Research Network. Macrolide antibiotics and the risk of cardiac arrhythmias. Am J Respir Crit Care Med 2014;189:1173-80.

190 Ikeda AK, Prince AA, Chen JX, et al. Macrolide-associated sensorineural hearing loss: a systematic review. Laryngoscope 2018;128:228-36.

191 Wallace MR, Miller LK, Nguyen MT, et al. Ototoxicity with azithromycin. Lancet 1994;343:241.

192 Tseng AL, Dolovich L, Salit IE. Azithromycin-related ototoxicity in patients infected with human immunodeficiency virus. Clin Infect Dis 1997;24:76-7.

193 Brown BA, Griffith DE, Girard W, et al. Relationship of adverse events to serum drug levels in patients receiving high-dose azithromycin for mycobacterial lung disease. Clin Infect Dis 1997;24:958-64.

194 Moseley RH. Macrolide antibiotics. Hepatotoxicity of antimicrobials and antifungal agents. In: Kaplowitz N, DeLeve LD, eds. Drug-induced liver disease. Amsterdam: Elsevier, 2013: 466-7.

195 Sundaram V, Björnsson ES. Drug-induced cholestasis. Hepatol Commun 2017;1:726-35.

196 HM Government. Contained and controlled: the UK's 20-year vision for antimicrobial resistance. Emergency Preparedness and Health Protection Policy Directorate, 2019.

197 World Health Organization. Global antimicrobial resistance surveillance system (GLASS) report: early implementation 2017-2018. World Health Organization, 2018.

198 Paterson DL. "Collateral damage" from cephalosporin or quinolone antibiotic therapy. Clin Infect Dis 2004;38(Suppl 4):S341-5.

199 Sivapalasingam S, Steigbigel NH. Macrolides, clindamycin and ketolides. In: Bennett JE, Dolin R, Blaser MJ, eds. Mandell, Douglas and Bennett's principle and practice of infectious diseases. 8th edn. Philadelphia: Elsevier Saunders, 2015: 358-76.

200 Gordon C. Azithromycin. In: Grayson M, ed. Kucers' The use of antibiotics. 7th edn. Boca Raton: CRC Press, 2018: 1122-49.

201 Leclercq R, Courvalin P. Intrinsic and unusual resistance to macrolide, lincosamide, and streptogramin antibiotics in bacteria. Antimicrob Agents Chemother 1991;35:1273-6.

202 Leclerca R, Courvalin P. Resistance to macrolides and related antibiotics in Streptococcus pneumoniae. Antimicrob Agents Chemother 2002;46:2727-34.

203 Schroeder MR, Stephens DS. Macrolide resistance in Streptococcus pneumoniae. Front Cell Infect Microbiol 2016;6:98

204 Bergman M, Huikko S, Huovinen P, et al. Macrolide and azithromycin use are linked to increased macrolide resistance in Streptococcus pneumoniae. Antimicrob Agents Chemother 2006;50:3646-50.
205 Farrell DJ, File TM, Jenkins SG. Prevalence and antibacterial susceptibility of mef(A)positive macrolide-resistant Streptococcus pneumoniae over 4 years (2000 to 2004) of the PROTEKT US Study. J Clin Microbiol 2007;45:290-3.

206 Imöhl M, Reinert RR, van der Linden M. Antibiotic susceptibility rates of invasive pneumococci before and after the introduction of pneumococcal conjugate vaccination in Germany. Int J Med Microbiol 2015;305:776-83.

207 Lowbury EJ, Hurst L. The sensitivity of staphylococci and other wound bacteria to erythromycin, oleandomycin, and spiramycin. J Clin Pathol 1959;12:163-9.

208 Seppälä H, Klaukka T, Vuopio-Varkila J, et al. The effect of changes in the consumption of macrolide antibiotics on erythromycin resistance in group $\mathrm{A}$ streptococci in Finland. Finnish Study Group for antimicrobial resistance. N Engl J Med 1997:337:441-6.

209 Haight TH, Finland M. Laboratory and clinical studies on erythromycin. N Eng/ J Med 1952;247:227-32.

210 Hare KM, Grimwood K, Chang AB, et al. Nasopharyngeal carriage and macrolide resistance in Indigenous children with bronchiectasis randomized to long-term azithromycin or placebo. Eur J Clin Microbiol Infect Dis 2015;34:2275-85.

211 Li H, Liu D-H, Chen L-L, et al. Meta-analysis of the adverse effects of long-term azithromycin use in patients with chronic lung diseases. Antimicrob Agents Chemother 2014;58:511-7.

212 Cilloniz C, Albert RK, Liapikou A, et al. The effect of macrolide resistance on the presentation and outcome of patients hospitalized for Streptococcus pneumoniae pneumonia. Am J Respir Crit Care Med 2015;191:1265-72.

213 Griffith DE, Aksamit T, Brown-Elliott BA, et al. An official ATS/IDSA statement: diagnosis, treatment, and prevention of nontuberculous mycobacterial diseases. Am J Respir Crit Care Med 2007;175:367-416.

214 Haworth CS, Banks J, Capstick T, et al. British Thoracic Society guideline for the management of non-tuberculous mycobacterial pulmonary disease (NTM-PD). BMJ Open Respir Res 2017;4:e00242.

215 Centers for Disease Control and Prevention. Sexually transmitted diseases treatment guidelines, 2015. Available: https://www.cdc.gov/std/tg2015/toc.htm

216 Public Health England. UK case of Neisseria gonorrhoeae with high-level resistance to azithromycin and resistance to ceftriaxone acquired abroad. 12. Health Protection Report, 2018.

217 Harris JB RE. Enteric fever and other causes of fever and abdominal symptoms. In: Bennett JE DR, Blaser MJ, eds. Mandell, Douglas and Bennett's principle and practice of infectious diseases. 8th edn. Philadelphia: Elsevier Saunders, 2015: 1270-82.

218 PHE. Salmonella typhi resistant to third-generation cephalosporins isolated in England from a traveller returning from Pakistan. Contract No: Health Protection Report 11. PHE, 2017

219 Fifer H, Saunders J, Soni S, et al. British Association for Sexual Health and HIV national guideline for the management of infection with Neisseria gonorrhoeae. BASHH, 2019.

220 WHO. The selection and use of essential medicines: report of the 21st WHO Expert Committee on the selection and use of essential medicines. Geneva: WHO, 2017.

221 Slimings C, Riley TV. Antibiotics and hospital-acquired Clostridium difficile infection: update of systematic review and meta-analysis. J Antimicrob Chemother 2014:69:881-91.

222 Korpela K, Salonen A, Virta LJ, et al. Intestinal microbiome is related to lifetime antibiotic use in Finnish pre-school children. Nat Commun 2016;7:10410.

223 Cho I, Yamanishi S, Cox L, et al. Antibiotics in early life alter the murine colonic microbiome and adiposity. Nature 2012;488:621-6.

224 Jernberg C, Löfmark S, Edlund C, et al. Long-term ecological impacts of antibiotic administration on the human intestinal microbiota. Isme J 2007;1:56-66.

225 Donath E, Chaudhry A, Hernandez-Aya LF, et al. A meta-analysis on the prophylactic use of macrolide antibiotics for the prevention of disease exacerbations in patients with chronic obstructive pulmonary disease. Respir Med 2013;107:1385-92.

226 Herath SC, Poole P. Prophylactic antibiotic therapy for chronic obstructive pulmonary disease (COPD). Cochrane Database Syst Rev 2013:CD009764.

227 Simoens S, Laekeman G, Decramer M. Preventing COPD exacerbations with macrolides: a review and budget impact analysis. Respir Med 2013;107:637-48.

228 Yao G-Y, Ma Y-L, Zhang M-Q, et al. Macrolide therapy decreases chronic obstructive pulmonary disease exacerbation: a meta-analysis. Respiration 2013;86:254-60.

229 Lee JS, Park D-A, Hong Y, et al. Systematic review and meta-analysis of prophylactic antibiotics in COPD and/or chronic bronchitis. Int J Tuberc Lung Dis 2013;17:153-62.

230 Seemungal TA, Donaldson GC, Paul EA, et al. Effect of exacerbation on quality of life in patients with chronic obstructive pulmonary disease. Am J Respir Crit Care Med 1998;157:1418-22. 


\begin{tabular}{|c|c|c|c|}
\hline $\begin{array}{l}\text { Supplementary 1: } \\
\text { Quick reference guide for } \\
\text { azithromycin }\end{array}$ & Asthma & & Bronchiectasis \\
\hline $\begin{array}{l}\text { Identify if suitable for } \\
\text { Azithromycin therapy }\end{array}$ & $\begin{array}{l}\text { Confirmed diagnosis of asthma } \\
\text { Symptomatic despite }>800 \mathrm{mcg} / \mathrm{BED} \\
\text { At least } 1 \text { exacerbation in previous } 12 \text { months } \\
\text { Inhaled therapies optimised including inhaler technique and } \\
\text { adherence review }\end{array}$ & $\begin{array}{l}\text { Confirmed diagnosis of COPD } \\
3 \text { or more exacerbations in previous } 12 \text { months OR } \\
1 \text { or more severe exacerbation with hospitalisation/morbidity } \\
\text { Inhaled therapies optimised including inhaler technique and } \\
\text { adherence review, smoking cessation and pulmonary } \\
\text { rehabilitation completed }\end{array}$ & $\begin{array}{l}\text { Confirmed diagnosis of bronchiectasis } \\
3 \text { or more exacerbations in previous } 12 \text { months } \\
\text { Optimisation of other interventions such as airway clearance } \\
\text { and pulmonary rehabilitation }\end{array}$ \\
\hline $\begin{array}{l}\text { Identify } \\
\text { Contra-indications } \\
\text { to macrolide therapy }\end{array}$ & $\begin{array}{l}\text { Absolute Contra-indication: } \\
\text { Previous allergy/intolerance to macrolides } \\
\text { History of prolonged QTc } \\
\text { Active NTM disease }\end{array}$ & & $\begin{array}{l}\text { Relative Contra-indications: } \\
\text { Hearing or balance problems } \\
\text { History of NTM disease } \\
\text { Abnormal liver function tests }\end{array}$ \\
\hline $\begin{array}{l}\text { Perform safety } \\
\text { checks before starting } \\
\text { therapy }\end{array}$ & $\begin{array}{l}\text { Baseline ECG- } \\
\text { If QTc prolonged (>450msec for men, }>470 \mathrm{msec} \text { for women) do } \\
\text { not give macrolide } \\
\text { Baseline liver function tests }\end{array}$ & $\begin{array}{l}\text { Standard sputum for baseline culture if able to expectorate } \\
\text { If bronchiectatic or clinical concern of NTM infection investigate to } \\
\text { exclude (following BTS guideline on NTM disease). }\end{array}$ & Review concomitant medications for potential interactions \\
\hline $\begin{array}{l}\text { Start Azithromycin } \\
\text { therapy }\end{array}$ & $\begin{array}{l}\text { Azithromycin }(250 \mathrm{mg} / 500 \mathrm{mg}) \text { thrice weekly } \\
\text { Plan to treat for } 6-12 \text { months } \\
\text { Warn of potential side effects }\end{array}$ & $\begin{array}{l}\text { Azithromycin } 500 \mathrm{mg} \text { thrice weekly or } 250 \mathrm{mg} \text { daily } \\
\text { Plan to treat for } 6-12 \text { months } \\
\text { Warn of potential side effects }\end{array}$ & $\begin{array}{l}\text { Azithromycin } 500 \mathrm{mg} \text { thrice weekly or } 250 \mathrm{mg} \text { daily } \\
\text { Plan to treat for } 6-12 \text { months } \\
\text { Warn of potential side effects }\end{array}$ \\
\hline $\begin{array}{l}\text { Monitoring during } \\
\text { therapy }\end{array}$ & $\begin{array}{l}\text { Liver function tests at } 1 \text { month and every } 6 \text { months } \\
\text { Repeat ECG at } 1 \text { month }- \text { if QTc prolonged (>450msec for men, } \\
>470 \text { msec for women) stop macrolide }\end{array}$ & $\begin{array}{l}\text { Enquire about side effects, especially Gl upset and hearing and } \\
\text { balance problems } \\
\text { Standard sputum for culture at review if able to expectorate }\end{array}$ & $\begin{array}{l}\text { Medication review for potential drug interactions } \\
\text { and QT prolongation }\end{array}$ \\
\hline $\begin{array}{l}\text { Review therapy at } \\
6-12 \text { months }\end{array}$ & $\begin{array}{l}\text { Objective evidence of improvement: } \\
\text { Reduction in exacerbation rate } \\
\text { Improvement in symptoms } \\
\text { Change in sputum microbiology including NTM growth } \\
\text { Medication review for potential interactions }\end{array}$ & $\begin{array}{l}\text { Objective evidence of improvement: } \\
\text { Reduction in exacerbation rate } \\
\text { Improvement in symptoms, QoL or CAT score } \\
\text { Change in sputum microbiology including NTM growth } \\
\text { Medication review for potential interactions }\end{array}$ & $\begin{array}{l}\text { Objective evidence of improvement: } \\
\text { Reduction in exacerbation rate } \\
\text { Improvement in symptoms, QoL } \\
\text { Change in sputum microbiology including NTM growth } \\
\text { Medication review for potential interactions }\end{array}$ \\
\hline $\begin{array}{l}\text { Decide if suitable for } \\
\text { ongoing therapy }\end{array}$ & Perform individual risk/benefit analysis & $\begin{array}{l}\text { If therapy continued ensure ongoing monitoring and annual review } \\
\text { of therapy }\end{array}$ & $\begin{array}{l}\text { Consider treatment break for 3-6 months each year to reduce } \\
\text { treatment burden (and possibly reduce microbiological } \\
\text { resistance) }\end{array}$ \\
\hline
\end{tabular}




\section{Appendix 1 - Guideline Group Members}

Dr Ingrid Du Rand (Co-Chair)

Dr David Smith (Co-Chair)

Dr Charlotte Addy

Dr Tim Collyns (microbiologist)

Dr Simon Hart

Dr Phil Mitchelmore

Professor Najib Rahman

Ms Ravijyot Saggu (pharmacist)

Mrs Joan McCarthy was the lay representative (October 2016-December 2017) 


\section{Appendix 2 - Macrolide Guideline Stakeholder Organisations}

Royal College of Physicians

Royal College of Physicians of Edinburgh

Royal College of Physicians and Surgeons of Glasgow

Royal College of General Practitioners

Royal College of Pathologists

Association of Respiratory Nurse Specialists

Royal College of Nursing

British Society for Haematology

British Lung Transplantation Society

UK Clinical Pharmacy Association Respiratory Group

Association for Palliative Medicine

Primary Care Respiratory Society

Association of Chartered Physiotherapists in Respiratory Care

Chartered Society of Physiotherapy

Association for Respiratory Technology and Physiology 


\section{Appendix 3 - Drugs that prolong the QT interval}

Antimicrobials
Moxifloxacin
Fluconazole
Ketoconazole
Antiarrhythmics
Dronedarone
Sotalol
Quinidine
Amiodarone
Flecainide
Antipsychotics
Risperidone
Fluphenazine
Haloperidol
Pimozide
Chlorpromazine
Quetiapine
Clonazepam

Antidepressants

Citalopram/escitalopram

Amitryptaline

Clomipramine

Dosulepin

Doxepin

Imipramine

Lofepramine

\section{Antiemetics}

Domperidone

Droperidol

Odansetron/Granesitron

\section{Others}

Methadone

Some antimalarials

Some antiretrovirals

This list is not exhaustive. Readers are guided to reference texts such as the BNF for complete listings of individual agents. 
Appendix 4

Measuring the QT interval

The QT interval is measured from the start of the Q wave to the end of the T wave. The QT interval varies inversely with heart rate, increasing as the rate slows and decreasing as the rate increases. The corrected QT interval (QTC) estimates the value of QT at a standard rate of 60 beats per minute. There are a variety of methodologies for arriving at a value for QTc from an ECG at a different heart rate.

There are free smartphone applications (such as MedCaIX) and websites (such as mdcalc.com) that will generate a value for QTc from QT interval and heartrate (RR interval)

QTC is prolonged if $>450 \mathrm{~ms}$ in men or $>470 \mathrm{~ms}$ in women

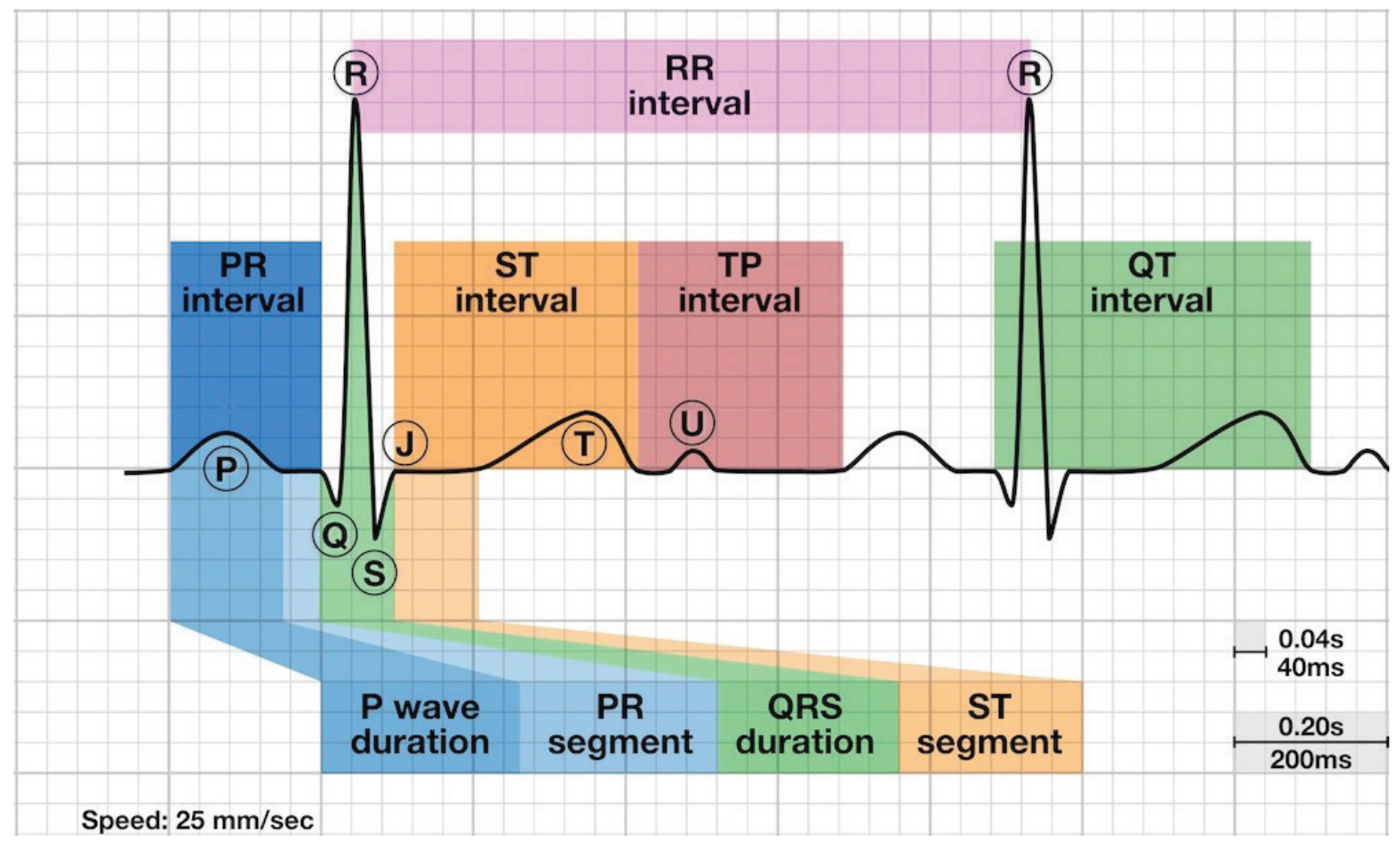

Graphic from "Life in the fast lane" blog (litfl.com)

Reproduced under the Attribution-NonCommercial-ShareAlike 4.0 International (CC BY-NC-SA 4.0)

https://litfl.com/qt-interval-ecg-library/

Dr Ed Burns

Accessed 26/11/2018 